\title{
Quantum simulation of interacting high-dimensional systems: the influence of noise
}

\author{
Wolfgang Dür ${ }^{1,2}$, Michael J. Bremner ${ }^{1,2}$ and Hans J. Briegel ${ }^{1,2}$ \\ 1 Institut für Theoretische Physik, Universität Innsbruck, Technikerstraße 25, A-6020 Innsbruck, Austria \\ ${ }^{2}$ Institut für Quantenoptik und Quanteninformation der Österreichischen Akademie der Wissenschaften, Innsbruck, Austria.
}

(Dated: November 3, 2018)

\begin{abstract}
We consider the simulation of interacting high-dimensional systems using pairwise interacting qubits. The main tool in this context is the generation of effective many-body interactions, and we examine a number of different protocols for obtaining them. These methods include the usage of higher-order processes (commutator method), unitary conjugation or graph state encoding, as well as teleportation based approaches. We illustrate and compare these methods in detail and analyze the time cost for simulation. In the second part of the article, we investigate the influence of noise on the simulation process. We concentrate on errors in the interaction Hamiltonians and consider two generic noise models, (i) timing errors in pairwise interactions and (ii) noisy pairwise interactions described by Master equations of Lindblad form. We analyze and compare the effect of noise for the different simulation methods and propose a way to significantly reduce the influence of noise by making use of entanglement purification together with a teleportation based protocol.
\end{abstract}

PACS numbers: 03.67.-a,03.67.Mn,03.67.Pp

Contents

1. Simulation protocol

14

I. Introduction

PART I: Methods to generate many-body interaction Hamiltonians
III. Unitary conjugation and graph state encoding

A. Graph-state encoding

B. Example 1: Three-body interaction Hamiltonian exhibiting a quantum phase transition

C. Example 2: Interacting $d$-dimensional systems

D. Example 3: 2D setup with 4-body plaquette interaction

E. Time cost and universal quantum simulation 9

1. Fixed graph state encoding:

2. Variable graph state encoding

IV. Teleportation based methods

A. The Jamiołkowski Isomorphism

Commutator method

A. Three-body interactions

B. Many-body interactions

1. The Jamiołkowski isomorphism for many-body systems

B. Teleportation-based gates using weakly entangled states

1. Quantum control and the Jamiołkowski isomorphism

2. Using weakly entangled states to generate phase gates

Teleportation-based gates using GHZ-type states

\section{PART II: Influence of noise}

V. Distance measures and fidelity

A. Jamiołkowski fidelity

B. Simple bounds on the Jamiołkowski fidelity for simulation protocols

C. Gate fidelity for long-time evolutions

VI. Noise model 1: Master equation

A. Local noise equivalent

VII. Noise model 2: Timing errors

1. Unitary Hamiltonians

2. Sums of commuting unitary Hamiltonians

VIII. Commutator method

A. Three-body interactions

B. Timing errors

C. Many-body interactions

D. Simulation of strongly entangling multi-qubit gate

E. Simulation of quDits using qudits

IX. Unitary conjugation and graph state encoding

A. Fixed graph state encoding

1. Three-body interaction

2. Many-body interaction

3. Timing errors

B. Variable graph state encoding

X. Teleportation based methods

A. Simulation of low dimensional systems using high dimensional systems
B. Perfect single-system operations

1. Three-body interactions 33 
2. Many-body interactions

3. Timing errors

C. Entanglement purification and the influence of local noise

1. Three-body interactions

2. Many-body interactions

\section{Comparison of methods}

\section{Summary and conclusions}

\section{Acknowledgements}

References

\section{INTRODUCTION}

Quantum computers are an example of a wide class of quantum-control systems that are capable of simulating the Hamiltonian dynamics of any finite-dimensional system. In the field of quantum information theory such systems are called universal quantum simulators, a name first coined by Feynman a quarter of a century ago to describe a class of physical systems whose dynamics can be manipulated in order to mimic the dynamics of any other system [1]. Feynman suggested that such systems might be able to overcome the seemingly insurmountable problem of efficiently simulating quantum mechanical systems. Meanwhile it has been shown that universal quantum computers, and all systems that can efficiently simulate quantum computers, are capable of efficiently simulating the dynamics of all finite-dimensional $k$-local Hamiltonians 2] and all sparse Hamiltonians [3, 4]. These classes of Hamiltonians include the set of all local spin systems and all Hamiltonians that can be efficiently mapped to such systems.

More specifically, we say that a quantum-control system is a universal quantum simulator on a set of $n$ subsystems if, under ideal conditions, it is capable of generating any unitary operation on that set of subsystems. There are many physical systems that can satisfy this condition given the right forms of control, for instance an "always-on" two-body Hamiltonian that entangles a set of $n$ qubits can be thought of as a universal quantum simulator if is supplemented by arbitrarily fast singlequbit unitary control [5]. These systems can also be considered to be universal quantum computers because they can efficiently simulate the quantum circuit model (see [6, 7, 8, 9, 10, 11, 12, 13, 14, 15, 16] for other examples involving Hamiltonians manipulated by single-qubit control). This form of quantum simulation is often referred to as Hamiltonian simulation, as it involves the manipulation of a fixed system Hamiltonian. Such universal quantum simulators are appealing from an experimental perspective because they have the prospect of utilizing "global" operations which can sometimes be more easily manipulated and created than quantum gates. Other, more direct approaches are concerned with simulation of specific interaction Hamiltonians (e.g. certain model Hamiltonians for high $T_{c}$ superconductivity) using quantum optical systems such as neutral atoms stored in an optical lattice [17, 18, 19]. This form of quantum simulator, while not universal, are more experimentally feasible in the short term.

Although many universal quantum simulators are equivalent to a full scale universal quantum computer, it is expected and hoped for that even without using complex quantum error correction or fault tolerant methods, one can simulate specific (relevant) Hamiltonians with sufficiently high accuracy, and gain in this way new insight in the corresponding systems. In particular, quantum simulators operating on a few tens of spins can be expected to be realized on a much shorter timescale than a fault tolerant universal quantum computer that needs to operate on hundreds of thousand spins.

With this in mind, in this article we undertake a study into the effects of noise on a number of different simulation protocols. The aims of our investigation are twofold:

(i) We introduce and investigate different methods to generate many-body interactions from two-body interactions with help of local control operations.

(ii) We study the influence of noise in two-body interaction and local control operations on the simulation process.

We concentrate on the simulation of many-body interaction Hamiltonians from two-body interaction Hamiltonians. Despite the fact that many-body interactions are often neglected in the theoretical description of quantum systems as they mostly appear as higher order processes, such terms play a pivotal role in the theory of quantum simulation. This becomes particularly apparent when one uses a system of qubits to simulate higherdimensional systems. For instance, if one wanted to simulate any eight dimensional spin system with three qubits then one would have to be able to simulate three-qubit interaction terms. A more extreme example of this is shown in 20, 21] where it is demonstrated that Fermionic systems can be simulated using interacting spin systems. Such simulations utilize a generalization of the Jordan-Wigner transformation 22] to demonstrate how Fermionic Hamiltonians can be mapped to a spin Hamiltonian which contains many-body interaction terms. Another application of many-body interaction Hamiltonians is in quantum error correcting protocols for adiabatic quantum computing. Although it is known that twolocal Hamiltonians are universal for adiabatic quantum computing 23], such schemes are not fault-tolerant. For existing error correction protocols it seems that, in order to correct errors in an adiabatic algorithm that uses a two-local Hamiltonian, we are required to use manybody interaction Hamiltonians [24].

In this paper we particularly focus on the effects of noise on protocols for simulating many-body interaction terms that are tensor products of the Pauli matrices. 
Such simulations, while having a simple form, are interesting because they can often be simulated via protocols that can be highly parallelized and they can be used to form the building blocks of more sophisticated protocols. In addition, such Hamiltonians can transform local noise into highly non-local noise. Our approach in this article is to examine the effects of noise on the entangling operations used in these protocols. The noise models that we study are quite general and can applied to a variety of physical implementations and, in principle, the analysis that we perform can be extended to more complex simulations.

More generally, the challenge ahead in the study of quantum simulators is to more precisely identify where the limitations lie. This problem has two clear pathways, the first being to identify the algorithmic constraints on quantum simulation. For instance, it is known that simulating the static properties of quantum systems seems to be a computationally difficult task because the problem of identifying whether or not a state of a two-local Hamiltonian is the ground state of that Hamiltonian is QMAcomplete [23] (the quantum analogue of NP-complete). Thus it is thought that this task is not efficiently solvable by a quantum computer or any quantum simulator that is computationally equivalent to a quantum computer. In spite of such results, there are currently few proven restrictions on the class of systems that are efficiently solvable on a quantum computer or an equivalent quantum simulator. In particular, much work needs to be done to identify physical systems that can be efficiently simulated by a universal quantum simulator and which quantities of these systems can be efficiently extracted. For example, recent work 25] has highlighted that there are subtle algorithmic conditions that must be satisfied in order to ensure that a quantum simulation protocol will achieve any speed-up over a classical simulation.

The second pathway is to identify the physical restrictions to our capacity to perform quantum simulations, which is the main focus of this article. If a quantum computer endures too much noise [26], its dynamics can be classically simulated, the same is true for all quantum simulators though the amount of noise that is too much for a general quantum simulator is not yet known. One way of approaching the problem of how much noise can be tolerated by a quantum simulator is to address the problem of how to eradicate noise in a simulation. Quantum computers can be made fault-tolerant through the use of error-correcting codes. The fault tolerance theorem tells us that that a noisy quantum computer employing quantum error correction can simulate an ideal quantum computer without too much additional overhead given that amount of noise is below a particular threshold and that the errors that occur are of the right kind (for a summary see e.g. chapter 10 of [27]). The key message of the threshold theorem is that it is in principle possible to build quantum simulation devices that can overcome the effects of noise and that any fault-tolerant quantum computing architecture is an example of such a device.
Currently, it is not known how far the theory of faulttolerant quantum computing can be extended. The first versions of the fault-tolerance theorem applied only to architectures that implement the circuit model of quantum computing, however, the theorem has since been extended to one-way models of quantum computing [28, 29, 30, 31, 32, 33. As of yet, there is not a complete understanding of how to develop a theory of fault-tolerance for systems that are controlled by adiabatic evolutions and for general time-varying Hamiltonian evolutions. That said, in [34] the authors demonstrated that the fault-tolerance theorem can be extended to account for systems enduring certain forms of nonMarkovian noise by considering a model of quantum computation where circuits are simulated by a "local" timevarying Hamiltonian, proving that such Hamiltonian control systems are also fault-tolerant. In addition to this there have been promising recent developments towards developing a theory of quantum error correction for adiabatic models of quantum computing [24].

So far we have discussed quantum simulators in the context of fault-tolerant quantum computers, yet it is far from clear as to whether quantum simulators have to be fault-tolerant to be useful. Current fault-tolerance thresholds for quantum computers with reasonable noise models lie between $10^{-5}$ and $10^{-3}$. While theoretical advances may raise these thresholds, current belief is it will not be possible to build any quantum computer that satisfies a fault-tolerant threshold for many years. As such, it is worthwhile to ask what can be done with systems that contain too much noise to be made fault-tolerant and yet by many measures are still highly quantum [35]? Such systems can simulate classical computations efficiently as well as a limited class of quantum systems that one may not be able to classically simulate. With the present paper, where we investigate the influence of noise on the simulation of many-body interactions, we aim to shed some light on the possibility of using quantum simulators in such an intermediate regime.

The paper is organized as follows. In part I, we investigate several methods to generate many-body interactions. In Sec. III we describe the standard commutator method that makes use of the Lie-Trotter product expansion. In Sec. III we introduce a method that is based on unitary conjugation or graph state encoding, while we consider teleportation based methods in Sec. IV] Part II of the article is concerned with a detailed study of the influence of noise in interactions on the simulation process. In Sec. $\mathrm{V}$ we discuss distance measures and fidelity of noisy processes. In Sec. VI we describe our first noise model, where noisy interactions are described by master equations of Lindblad form. We consider a second noise model taking random fluctuations in interaction time into account in Sec. VII, In Sections VIII, IX and $\mathrm{X}$ we investigate in detail the influence of noise on the simulation process for commutator method, graph state encoding and teleportation based method respectively. In Sec. X we also discuss the usage of entanglement purification to 
significantly reduce the influence of noise. The results for the different methods are compared in Sec. XI, and we summarize in Sec. XII.

\section{PART I: Methods to generate many-body interaction Hamiltonians}

We will consider $m$ systems, each of dimension $d$, with associated Hilbert space $\mathcal{H}=\left(\mathbb{C}^{d}\right)^{\otimes m}$. In the case of $d=2$, i.e. qubits, we make use of the Pauli-matrices which we denote by $\sigma_{0} \equiv \mathbb{1}, \sigma_{1} \equiv \sigma_{x}, \sigma_{2} \equiv \sigma_{y}, \sigma_{3} \equiv \sigma_{z}$. When it is clear from the context, we will often omit tensor products, i.e. we identify $\sigma_{i}^{(A)} \otimes \sigma_{j}^{(B)} \equiv \sigma_{i}^{(A)} \sigma_{j}^{(B)}$. We will also set $\hbar=1$ in the following.

We assume that the $d$-level systems interact pairwise, and we will be interested in methods to generate effective many-body interactions involving up to $n \leq m$ of these systems. For simplicity, we will mainly consider $d=2$, i.e. qubits. The simulation of arbitrary manybody Hamiltonians of $m$ qubits (or, equivalently, Hamiltonians of higher dimensional systems with $D=2^{m}$ ) can be achieved if one is capable of generating

(i) a specific many-body interaction Hamiltonian $H=$ $\sigma_{z}^{\otimes n}$ for all $n \leq m$

(ii) fast local unitary control of the individual qubits.

Hence we will concentrate on the following in methods to generate a basic $m$-body interaction for some fixed $m$.

Given (i) and (ii) are fulfilled, standard techniques from Hamiltonian simulation can be applied, where intermediate fast local unitary operations are used to manipulate the basic Hamiltonian $H$ and generate an arbitrary desired effective Hamiltonian

$$
H^{\prime}=\sum_{k} \lambda_{k} H_{k},
$$

where $H_{k}$ are $m$-body interaction Hamiltonians consisting of Pauli matrices. Here, one makes use of the facts that any $m$-body Hamiltonian can be represented in the Pauli basis, and Hamiltonians $H_{k}$ consisting of Paulimatrices can effectively generated from $H$ via unitary conjugation, i.e.

$$
U_{k} e^{-i t H} U_{k}^{\dagger}=e^{-i t U_{k} H U_{k}^{\dagger}},
$$

where local unitary operations $U_{k}$ suffice. Using the identity

$$
\lim _{M \rightarrow \infty}\left(\prod_{k} e^{-i H_{k} t / M}\right)^{M}=e^{-i t \sum_{k} H_{k}},
$$

we find for sufficiently short times $t=\delta t$,

$$
\begin{aligned}
\prod_{k} e^{-i \delta t \lambda_{k} H_{k}} & =\mathbb{1}-i \delta t \sum_{k} \lambda_{k} H_{k}+O\left(\delta t^{2}\right) \\
& \approx e^{-i \delta t \sum_{k} \lambda_{k} H_{k}}
\end{aligned}
$$

that is, sequences of applications of the standard Hamiltonian $H$, together with intermediate local unitary operations $U_{k}^{\dagger} U_{k+1}$, generate (up to higher order corrections in $\delta t$ ) a unitary operation that is generated by an effective Hamiltonian $H^{\prime}$ (Eq. 1). Note that more complicated sequences allow for the simulation of the effective Hamiltonian $H^{\prime}$ with higher accuracy, with corrections appearing only in higher order $\delta t$.

\section{COMMUTATOR METHOD}

\section{A. Three-body interactions}

A somewhat standard approach to simulating a three body interaction from a given two-body interaction is to apply a sequence of time evolutions, generated by different Hamiltonians, each for a short time $\delta t$. The sequence is chosen in such a way that all first order terms in $\delta t$-when performing a Taylor expansion-cancel, and only higher order terms in $\delta t$ remain. These higher order terms include products of different two-body Hamiltonians, and can hence correspond to effective many-body interactions. To be more precise, consider the following sequence of time evolutions, generated by the Hamiltonians $H_{k}$ applied for time $\delta t$

$$
U_{\text {tot }}=e^{i H_{4} \delta t} e^{i H_{3} \delta t} e^{i H_{2} \delta t} e^{i H_{1} \delta t} .
$$

For small $\delta t$, one can Taylor expand this expression and obtains

$$
\begin{aligned}
U_{\text {tot }}= & \mathbb{1}+i \delta t \sum_{j=1}^{4} H_{j} \\
& -\frac{\delta t^{2}}{2}\left(\sum_{j=1}^{4} H_{j}^{2}+2 \sum_{l=2}^{4} \sum_{j=1}^{l-1} H_{l} H_{j}\right) \\
& +O\left(\delta t^{3}\right)
\end{aligned}
$$

Considering the case

$$
H_{4}=-H_{2}, \quad H_{3}=-H_{1},
$$

one obtains

$$
\begin{aligned}
U_{\mathrm{tot}} & =\mathbb{1}+\delta t^{2}\left(H_{1} H_{2}-H_{2} H_{1}\right)+O\left(\delta_{t}^{3}\right) \\
& =e^{i 2 \delta t^{2} \times(-i / 2)\left[H_{1}, H_{2}\right]}+O\left(\delta t^{3}\right) .
\end{aligned}
$$

The sequence of interactions described by some properly chosen two-body Hamiltonians hence correspond -up to higher order corrections- to an evolution which is described by an Hamiltonian that is essentially given by

$$
H_{\mathrm{eff}}=-i / 2\left[H_{1}, H_{2}\right]
$$

which can be an effective three-body interaction.

Example: For a system of three qubits and

$$
\begin{aligned}
& H_{1}=\sigma_{z}^{(A)} \sigma_{x}^{(B)}, \\
& H_{2}=\sigma_{y}^{(B)} \sigma_{z}^{(C)},
\end{aligned}
$$


we have that

$$
\begin{aligned}
e^{\left[H_{1}, H_{2}\right] \delta t^{2}}+O\left(\delta t^{3}\right) \approx & \mathbb{1}^{(A B C)}+i 2 \delta t^{2} \sigma_{z}^{(A)} \sigma_{z}^{(B)} \sigma_{z}^{(C)} \\
& +O\left(\delta t^{3}\right) \\
\approx & e^{i \delta t^{\prime} H_{\mathrm{eff}}}+O\left(\delta t^{\prime 3 / 2}\right)
\end{aligned}
$$

That is, if we evolve a system as indicated in Eq. (5) using only two-body interactions with $H_{4}=-H_{2}, H_{3}=$ $-H_{1}$ for a total time of $4 \delta t$, then the resulting evolutions is - up to higher order corrections in $\delta t$ - the same as the one resulting from a three-body Hamiltonian

$$
H_{\mathrm{eff}}=\sigma_{z}^{(A)} \sigma_{z}^{(B)} \sigma_{z}^{(C)}
$$

applied for a time

$$
\delta t^{\prime}=2 \delta t^{2}
$$

Note that there is a dilation factor of $2 / \delta t$, i.e. the required physical time $t$ to implement an effective threebody interaction for a time $t^{\prime}$ is given by $t=4 \sqrt{\delta t^{\prime} / 2}$, since the effective three body interaction only appears in second order in $\delta t$. We have assumed that $H_{1}, H_{2}$ as well as $-H_{1},-H_{2}$ can be implemented. The simulation of $H_{\text {eff }}$ is only correct in first order in $\delta t^{\prime}$, and unwanted terms appear already in order $\delta t^{\prime 3 / 2}$. This corresponds to a reduced accuracy as compared to standard Hamiltonian simulation schemes for two-body interactions, where the desired Hamiltonian is correctly produced up to first order, and undesired terms (errors) appear only in second order. We will refer to this kind of errors as Taylor expansion errors, which are independent from errors in interactions and local control operations which will discussed in detail in Sec. IVC1. The Taylor expansion errors need to be taken into account when using the effective $m$-body Hamiltonian to simulate other Hamiltonians via Hamiltonian simulation techniques.

\section{B. Many-body interactions}

In principle, many-body interactions Hamiltonians for arbitrary number of qubits $m$ can be generated in a recursive way using above method. For instance, one of the two Hamiltonians, say $H_{1}$, is replaced by an effective $m-1$ body Hamiltonian. Together with an appropriate two-body Hamiltonian a new effective $m$-body Hamiltonian can be produced. Note, however, that there is a dilation factor of $\delta t / 2$ in each of these simulation processes. That is, the implementation of a $m$-body Hamiltonian for time $t^{\prime}$ requires a physical time $t=O\left(t^{2^{-(m-1)}}\right)$, if basic two-body Hamiltonians are used.

The time cost can be reduced when using an alternative method, where two $n$-body interactions are used to generate a $m=(2 n-1)$-body interaction. For instance, the generation of a 5 -body interaction has a time cost of $O\left(\delta t^{1 / 4}\right)$, as compared to $O\left(\delta t^{1 / 8}\right)$ when using the first method. The desired $m$-body Hamiltonian appears in $O\left(\delta t^{m-1}\right)$ and corrections appear in $O\left(\delta t^{m}\right)$, where $m=5$ in our example. Rewriting this in the new effective time $\delta t_{m}$, i.e. one realizes a $m$-body Hamiltonian for time $\delta t_{m}$, one finds

$$
\delta t_{m}=O\left(\delta t^{m-1}\right)
$$

and corrections appear in

$$
O\left(\delta t_{m}^{m /(m-1)}\right) .
$$

That is,

$$
\begin{aligned}
U_{\mathrm{tot}} & =\mathbb{1}+i O\left(\delta t^{m-1}\right) H_{\mathrm{eff}}+O\left(\delta t^{m}\right) \\
& =\mathbb{1}+i \delta t_{m} H_{\mathrm{eff}}+O\left(\delta t_{m}^{m /(m-1)}\right) \\
& \approx e^{i \delta t_{m} H_{\mathrm{eff}}}
\end{aligned}
$$

To ensure that the Taylor expansion is a good approximation at all instances of the protocol, all involved times, in particular $\delta t=O\left(\delta t_{m}^{1 /(m-1)}\right.$ ) (which corresponds to the physical time for which two-body interactions are applied), need to be sufficiently small. This limits the possible values of $\delta t_{m}$, which is important when considering the simulation of general $m$-body Hamiltonians from the basic one. In particular, the total time required to simulate a Hamiltonian for time $t_{\text {tot }}$ with Hamiltonian simulation techniques (i.e. generating an effective $m-$ body Hamiltonian for time $\delta t_{m}$, and using this Hamiltonian together with intermediate local unitary operations to simulate other Hamiltonians for larger times by repeating this process $t_{\text {tot }} / \delta t_{m}$ times) requires a total time of

$$
t_{\mathrm{tot}} / \delta t_{m} \times O\left(\delta t_{m}^{1 /(m-1)}\right)=t_{\mathrm{tot}} O\left(\delta t_{m}^{-(m-2) /(m-1)}\right) .
$$

The dilation factor (or time cost) $O\left(\delta t_{m}^{-(m-2) /(m-1)}\right)$ approaches $O\left(\delta t_{m}^{-1}\right)$ for large $m$ and can be significant. Recall that $\delta t_{m}^{1 /(m-1)} \ll 1$ needs to be fulfilled, which implies that the dilation factor, for large $m$, will typically be of order $10^{m}$ or larger.

Example: To make above considerations more concrete, consider the generation of an effective 5-body interaction Hamiltonian for time $\delta t^{\prime \prime}$,

$$
U_{\text {tot }}\left(\delta t^{\prime \prime}\right) \approx e^{-i \delta t^{\prime \prime} H_{\text {eff }}},
$$

where we use the notation $\delta t^{\prime \prime} \equiv \delta t_{5}, \delta t^{\prime} \equiv \delta t_{3}$. This could take place as follows: (i) use two-body interactions $U_{A B}( \pm \delta t), U_{B C}( \pm \delta t)$ generated by two-body Hamiltonians $\pm H_{A B}, \pm H_{B C}$ between systems $A B, B C$ for time $\delta t=\sqrt{\delta t^{\prime} / 2}$ to produce an effective three-body interaction $U_{A B C}\left(\delta t^{\prime}\right)$ generated by the effective three-body Hamiltonian $H_{A B C}=-i / 2\left[H_{B C}, H_{A B}\right]$. That is,

$$
\begin{aligned}
U_{A B C}\left(\delta t^{\prime}\right) & =U_{A B}(-\delta t) U_{B C}(-\delta t) U_{A B}(\delta t) U_{B C}(\delta t) \\
& \approx e^{i \delta t^{\prime} H_{A B C}} .
\end{aligned}
$$

Use the same method to produce a three-body interaction $U_{C D E}\left(\delta t^{\prime}\right)$ generated by the effective three-body 
Hamiltonian $H_{C D E}=\left[H_{D E}, H_{C D}\right]$. (ii) Use these threebody interactions $U_{A B C}\left(\delta t^{\prime}\right), U_{C D E}\left(\delta t^{\prime}\right)$ to produce an effective 5 -body interaction $U_{\text {tot }}\left(\delta t^{\prime \prime}\right)$ generated by the effective 5-body Hamiltonian $H_{\mathrm{eff}}=-i / 2\left[H_{C D E}, H_{A B C}\right]$ for time

$$
\delta t^{\prime \prime}=\sqrt{\delta t^{\prime} / 2}=(\delta t / 8)^{\frac{1}{4}} .
$$

That is,

$$
\begin{aligned}
U_{\text {tot }}\left(\delta t^{\prime \prime}\right) & =U_{A B C}\left(-\delta t^{\prime}\right) U_{C D E}\left(-\delta t^{\prime}\right) U_{A B C}\left(\delta t^{\prime}\right) U_{C D E}(\delta t) \\
& \approx e^{i \delta t^{\prime \prime} H_{\text {eff }}} .
\end{aligned}
$$

The choice

$$
\begin{array}{ll}
H_{A B}=\sigma_{z}^{(A)} \sigma_{x}^{(B)}, & H_{B C}=\sigma_{y}^{(B)} \sigma_{y}^{(C)}, \\
H_{C D}=\sigma_{x}^{(C)} \sigma_{y}^{(D)}, & H_{D E}=\sigma_{x}^{(D)} \sigma_{z}^{(E)},
\end{array}
$$

leads to an effective 5-body Hamiltonian

$$
H_{\mathrm{eff}}=\sigma_{z}^{\otimes 5} .
$$

\section{UNITARY CONJUGATION AND GRAPH STATE ENCODING}

A second method to generate effective many-body interaction Hamiltonians from basic two-body interaction Hamiltonians is by unitary conjugation. That is, before [after] the evolution with respect to a single- or two-body Hamiltonian $H$, a (possibly non-local) unitary operation $U\left[U^{\dagger}\right]$ is applied. The resulting evolution is given by

$$
U e^{-i t H} U^{\dagger}=e^{-i t U H U^{\dagger}},
$$

i.e. by the transformed Hamiltonian $H^{\prime}=U H U^{\dagger}$. If $U$ is itself a non-local unitary operation (e.g. generated by two-body interactions), then the resulting effective Hamiltonian $H^{\prime}$ can contain many-body terms. The unitary conjugation can be viewed as a change of basis, where single- and two-body terms of an interaction Hamiltonian act effectively as many-body terms in the new basis. In the following, we will concentrate a specific family of basis changes, associated with graph states. The corresponding unitary operations $U$ can be efficiently implemented using basic two-body interactions, and allow for the systematic construction of the desired manybody Hamiltonians via a proper choice of the graph. This gives a powerful tool for the construction of many-body interaction Hamiltonians.

Consider as a first example a system of two qubits, and a unitary operation $U=U_{P G}$,

$$
U_{P G}=\operatorname{diag}(1,1,1,-1),
$$

i.e. a phase gate. A phase gate can e.g. be induced by a simple two-body Hamiltonian

$$
H=\left(\mathbb{1}-\sigma_{z}\right) \otimes\left(\mathbb{1}-\sigma_{z}\right),
$$

applied for time $t=\pi / 4$, which is locally equivalent to an Ising Hamiltonian $H=\sigma_{z} \otimes \sigma_{z}$. With this choice of $U$, operators of the form $\sigma_{i} \otimes \mathbb{1}$ are transformed to $U_{P G}\left(\sigma_{i} \otimes \mathbb{1}\right) U_{P G}^{\dagger}=\sigma_{i} \otimes \sigma_{f(i)}$, with $f(i)=0$ if $i=0,3$ and $f(i)=3$ if $i=1,2$. That is, a single-body Hamiltonian acting on the first qubit,

$$
H=\sum \lambda_{k} \sigma_{k} \otimes \mathbb{1},
$$

is transformed into a two-body Hamiltonian

$$
\begin{aligned}
H^{\prime} & =U_{P G} H U_{P G}^{\dagger} \\
& =\lambda_{0} \sigma_{0} \otimes \sigma_{0}+\lambda_{1} \sigma_{1} \otimes \sigma_{3}+\lambda_{2} \sigma_{2} \otimes \sigma_{3}+\lambda_{3} \sigma_{3} \otimes \sigma_{0} .
\end{aligned}
$$

We remark that the phase-gate corresponds to the unitary transformation from a product basis $\left|k_{1} k_{2}\right\rangle, k_{i}=0,1$ in the $x$-basis (i.e. $k_{i}=0$ corresponds to $|0\rangle_{x}=$ $\frac{1}{\sqrt{2}}\left(|0\rangle_{z}+|1\rangle_{z}\right) \equiv \frac{1}{\sqrt{2}}(|0\rangle+|1\rangle)$, and $\left.|1\rangle_{x}=\frac{1}{\sqrt{2}}(|0\rangle-|1\rangle)\right)$ into a Graph-state basis $\left|G, k_{1} k_{2}\right\rangle$, where $G$ is the graph with vertices 1,2 and a single edge, $E=\{1,2\}$. That is,

$$
\left|G, k_{1} k_{2}\right\rangle=U_{P G}\left|k_{1} k_{2}\right\rangle \text {. }
$$

\section{A. Graph-state encoding}

More generally, for systems of $N$ qubits one can define an encoding into a graph-state basis corresponding to an arbitrary graph $G$. In this case, the graph $G=(V, E)$, is a set $V$ of $N$ vertices connected by edges E, corresponds to an interaction pattern (specified by $E$ ) between the qubits (that are associated with vertices). That is,

$$
|G, \boldsymbol{k}\rangle=\prod_{\{\alpha, \beta\} \in E} U_{P G}^{(\alpha, \beta)}|\boldsymbol{k}\rangle,
$$

where $\boldsymbol{k}$ is a binary vector of length $N$. We define the neighborhood $N_{\alpha}$ of a given vertex $\alpha$ as the set of all vertices connected to $\alpha$ in the graph, $N_{\alpha}=\{\beta \mid\{\alpha, \beta\} \in$ $E\}$. We use the notation

$$
\sigma_{i}^{N_{\alpha}}=\otimes_{\alpha \in N_{\alpha}} \sigma_{i}^{(\alpha)},
$$

to refer to an operator acting on all qubits in the neighborhood of $\alpha$. Using a such graph state encoding, the corresponding unitary operation is given by

$$
U=\prod_{\{\alpha, \beta\} \in E} U_{P G}^{(\alpha, \beta)} .
$$

Note that the phase gates commute and can hence be implemented in parallel. It is now straightforward to determine the effect of unitary conjugation with such a $U$. Pauli operators acting on qubit $\alpha$ are transferred as follows [36]

$$
\begin{aligned}
& U \sigma_{0}^{(\alpha)} U^{\dagger}=\sigma_{0}^{(\alpha)}, \\
& U \sigma_{1}^{(\alpha)} U^{\dagger}=\sigma_{1}^{(\alpha)} \sigma_{3}^{N_{\alpha}}, \\
& U \sigma_{2}^{(\alpha)} U^{\dagger}=\sigma_{2}^{(\alpha)} \sigma_{3}^{N_{\alpha}}, \\
& U \sigma_{3}^{(\alpha)} U^{\dagger}=\sigma_{3}^{(\alpha)} .
\end{aligned}
$$


We have that $\sigma_{1}^{(\alpha)}$ and $\sigma_{2}^{(\alpha)}$ are transformed into effective $m$-body interaction terms, where $m$ is given by the local degree of the graph (i.e. the number of neighbors of $\alpha$ ) plus one. The action of $\sigma_{3}^{(\alpha)}$ remains local. The transformation of two-body terms follows immediately from Eq. (33), i.e.

$$
U \sigma_{i}^{(\alpha)} \otimes \sigma_{j}^{(\beta)} U^{\dagger}=\left(U \sigma_{i}^{(\alpha)} U^{\dagger}\right)\left(U \sigma_{j}^{(\beta)} U^{\dagger}\right) .
$$

Note that $\left(U \sigma_{i}^{(\alpha)} U^{\dagger}\right)$ and $\left(U \sigma_{j}^{(\beta)} U^{\dagger}\right)$ can contain terms that act on the same qubit which need to be multiplied. Depending on the neighborhood relation of qubits $\alpha$ and $\beta$, and on the kind of Pauli operators, the resulting total operator can have support on up to $\left|N_{\alpha}\right|+\left|N_{\beta}\right|+2$ (i.e. acts non-trivially on this number of qubits), but may also be the identity. For instance,

$$
\begin{aligned}
U \sigma_{1}^{(\alpha)} \otimes \sigma_{1}^{(\beta)} U^{\dagger} & =\sigma_{1}^{(\alpha)} \sigma_{1}^{(\beta)} \sigma_{3}^{N_{\alpha}} \sigma_{3}^{N_{\beta}} \\
& =\sigma_{1}^{(\alpha)} \sigma_{1}^{(\beta)} \sigma_{3}^{N_{\alpha} \oplus N_{\beta}},
\end{aligned}
$$

where $\oplus$ denotes the XOR (exclusive or operation), i.e. $N_{\alpha} \oplus N_{\beta} \equiv\left(N_{\alpha} \cup N_{\beta}\right) \backslash\left(N_{\alpha} \cap N_{\beta}\right)$.

To illustrate the transformation rules, we consider a simple example of three qubits arranged as an open chain, i.e. with edges $E=\{\{1,2\},\{2,3\}\}$ and corresponding unitary $U=U_{P G}^{(1,2)} U_{P G}^{(2,3)}$. The two-qubit operator $\sigma_{1}^{(1)} \sigma_{3}^{(2)}$ transforms to a single qubit operator $\sigma_{x}^{(1)}$, as $U \sigma_{1}^{(1)} U^{\dagger}=\sigma_{1}^{(1)} \sigma_{3}^{(2)}$ and $U \sigma_{3}^{(2)} U^{\dagger}=\sigma_{3}^{(2)}$. Similarly, a two qubit operator $\sigma_{1}^{(1)} \sigma_{1}^{(3)}$ is transformed to a two-qubit operator $\sigma_{1}^{(1)} \sigma_{1}^{(3)}$, while $\sigma_{1}^{(1)} \sigma_{3}^{(3)}$ transforms to a three-body operator $\sigma_{1}^{(1)} \sigma_{3}^{(2)} \sigma_{3}^{(3)}$. In this case, a three-body operator can also be obtained from a single qubit operator, e.g. $\sigma_{1}^{(2)}$ transforms into $\sigma_{3}^{(1)} \sigma_{1}^{(2)} \sigma_{3}^{(3)}$.

\section{B. Example 1: Three-body interaction Hamiltonian exhibiting a quantum phase transition}

We consider now the generation of a three-body interaction Hamiltonian with transversal magnetic field, where we assume a linear chain of qubits with periodic boundary conditions. We have

$$
H^{\prime}=\sum_{\alpha}\left(-\sigma_{3}^{(\alpha-1)} \sigma_{1}^{(\alpha)} \sigma_{3}^{(\alpha+1)}+B \sigma_{1}^{(\alpha)}\right) .
$$

This Hamiltonian has been considered in Ref. 37, 38, 39] in the context of optical lattices in a triangular configuration, where three-body processes may lead in certain parameter regimes to an interaction of this form. This Hamiltonian exhibits a quantum phase transition. More importantly, it has a finite energy gap above its unique ground state, a finite classical correlation length but despite of this an diverging entanglement length [40]. We remark that the ground state of this system for $B=0$ is a one-dimensional cluster state, i.e. a graph state corresponding to a closed linear chain.
We now show how to generate the Hamiltonian $H^{\prime}$ using only two-body interaction and a simple graph state encoding. To this aim, we consider a graph with edges $(2 \alpha, 2 \alpha+1)$. That is, every second qubit is connected with its right neighbor. We use this graph for our graph state encoding, i.e. define the unitary operation $U$ as

$$
U=\prod_{\alpha} U_{P G}^{(2 \alpha, 2 \alpha+1)} .
$$

It is now straightforward to check the following transformation rules

$$
\begin{aligned}
& U \sigma_{3}^{(2 \alpha-1)} \sigma_{1}^{(2 \alpha)} U^{\dagger}=\sigma_{3}^{(2 \alpha-1)} \sigma_{1}^{(2 \alpha)} \sigma_{3}^{(2 \alpha+1)}, \\
& U \sigma_{1}^{(2 \alpha-1)} \sigma_{3}^{(2 \alpha)} U^{\dagger}=\sigma_{3}^{(2 \alpha-2)} \sigma_{1}^{(2 \alpha-1)} \sigma_{3}^{(2 \alpha)}, \\
& U \sigma_{3}^{(2 \alpha)} \sigma_{1}^{(2 \alpha+1)} U^{\dagger}=\sigma_{1}^{(2 \alpha)} \\
& U \sigma_{1}^{(2 \alpha)} \sigma_{3}^{(2 \alpha+1)} U^{\dagger}=\sigma_{1}^{(2 \alpha+1)} .
\end{aligned}
$$

That is, the two-body Hamiltonian

$$
H_{1}=\sum_{\alpha}-\left(\sigma_{3}^{(2 \alpha-1)} \sigma_{1}^{(2 \alpha)}+\sigma_{1}^{(2 \alpha-1)} \sigma_{3}^{(2 \alpha)}\right)
$$

transforms into

$$
H_{1}^{\prime}=\sum_{\alpha}-\sigma_{3}^{(\alpha-1)} \sigma_{1}^{(\alpha)} \sigma_{3}^{(\alpha+1)},
$$

and gives the desired three--body interaction terms, while

$$
H_{2}=B \sum_{\alpha}\left(\sigma_{3}^{(2 \alpha)} \sigma_{1}^{(2 \alpha+1)}+\sigma_{1}^{(2 \alpha)} \sigma_{3}^{(2 \alpha+1)}\right)
$$

transforms to

$$
H_{2}^{\prime}=B \sum_{\alpha} \sigma_{1}^{(\alpha)}
$$

and provides the transversal magnetic field in $X$ direction. In total, the two-body Hamiltonian $H=$ $H_{1}+H_{2}$ is transformed via unitary conjugation with $U$ (37) into the desired Hamiltonian $H^{\prime}$ (36). That is, for all times $t, U e^{-i t\left(H_{1}+H_{2}\right)} U^{\dagger}=e^{-i t H^{\prime}}$. We remark that one can in addition achieve a magnetic field in $Z$ direction via a single-body Hamiltonian $\sum_{\alpha} \sigma_{3}^{(\alpha)}$ which is not changed by unitary conjugation.

We emphasize that the method proposed here to generate the three-body Hamiltonian may be significantly simpler and easier to implement in optical lattices than the original proposal of Ref. [37, 38, 39], as it does not rely on higher-order processes. We require, however, individual addressability of the individual qubits in some form. The encoding- and decoding pattern (i.e. the operation $U$ ) can directly be generated from the available two-body interaction with is essentially given by a pairwise Ising Hamiltonian $\sigma_{z} \otimes \sigma_{z}$ resulting e.g. from controlled cold collisions. The same interaction, together with fast local unitary control operations, can also be used to generate the Hamiltonian $H=H_{1}+H_{2}$ using Hamiltonian simulation techniques. That is, the simulation of the evolution with respect to the three-body Hamiltonian $H^{\prime}$ for time $t_{\text {tot }}$ takes place as follows: 
1. Use the basic two-body interactions to generate $U$;

2. Simulate the evolution with respect to the Hamiltonian $H=H_{1}+H_{2}$ for time $\delta t$ by using the basic two-body interaction together with fast local unitary operations. Here, standard Hamiltonian simulation techniques are applied, and the desired process is approximated up to corrections $O\left(\delta t^{2}\right)$.

3. Repeat the process $t_{\text {tot }} / \delta t$ times. For $\delta t$ sufficiently small the total evolution will be a high-fidelity approximation of the evolution generated by $H$ applied for time $t_{\text {tot }}$.

4. Use the basic two-body interactions to generate $U^{\dagger}=U$.

The total evolution after steps 1-4 corresponds to $e^{-i t_{\text {tot }} H^{\prime}}$ as desired.

\section{Example 2: Interacting $d$-dimensional systems}

We now consider the simulation of Hamiltonians corresponding to (interacting) $d$-dimensional systems. To illustrate this approach, we first consider a single $d-$ dimensional system with $d=2^{4}=16$. In contrast to the previous example, we will make use of auxiliary systems here. That is, we consider a system of five qubits $1,2,3,4, A$ and the associated graph $G$ with edges $\{k, A\}$, $k=1,2,3,4$, i.e. each of the qubits $1,2,3,4$ (which represent the 16-dimensional system) is connected to qubit $A$. Here, qubit $A$ serves as auxiliary system and is prepared in an eigenstate of $\sigma_{x}$ with eigenvalue +1 , i.e. in the state $|0\rangle_{x}=(|0\rangle+|1\rangle)_{A} / \sqrt{2}$. This ensures that any effective Hamiltonian that acts on qubit $A$ as either the identity $\mathbb{1}^{(A)}$ or $\sigma_{x}^{(A)}$, leaves this qubit invariant, i.e. qubit $A$ is decoupled from the evolution in this case. However, Hamiltonians involving qubit $A$ can be used to manipulate and trigger the effective Hamiltonian acting on system qubits 1 to 4 . The unitary operation $U$ corresponding to the graph state encoding is given by

$$
U=\prod_{k=1}^{4} U_{P G}^{(k, A)} .
$$

This leads to the following transformation rules of single- and two-body operators under unitary conjuga- tion, where $k, k_{i} \in\{1,2,3,4\}$

$$
\begin{aligned}
U \sigma_{1}^{(\alpha)} \sigma_{3}^{(A)} U^{\dagger} & =\sigma_{1}^{(\alpha)}, \\
U \sigma_{2}^{(\alpha)} \sigma_{3}^{(A)} U^{\dagger} & =\sigma_{2}^{(\alpha)}, \\
U \sigma_{3}^{(\alpha)} U^{\dagger} & =\sigma_{3}^{(\alpha)}, \\
U \sigma_{1}^{\left(\alpha_{1}\right)} \sigma_{1}^{\left(\alpha_{2}\right)} U^{\dagger} & =\sigma_{1}^{\left(\alpha_{1}\right)} \sigma_{1}^{\left(\alpha_{2}\right)}, \\
U \sigma_{1}^{\left(\alpha_{1}\right)} \sigma_{2}^{\left(\alpha_{2}\right)} U^{\dagger} & =\sigma_{1}^{\left(\alpha_{1}\right)} \sigma_{2}^{\left(\alpha_{2}\right)}, \\
U \sigma_{2}^{\left(\alpha_{1}\right)} \sigma_{2}^{\left(\alpha_{2}\right)} U^{\dagger} & =\sigma_{2}^{\left(\alpha_{1}\right)} \sigma_{2}^{\left(\alpha_{2}\right)}, \\
U \sigma_{3}^{\left(\alpha_{1}\right)} \sigma_{3}^{\left(\alpha_{2}\right)} U^{\dagger} & =\sigma_{3}^{\left(\alpha_{1}\right)} \sigma_{3}^{\left(\alpha_{2}\right)}, \\
U \sigma_{3}^{\left(\alpha_{1}\right)} \sigma_{3}^{(A)} U^{\dagger} & =\sigma_{3}^{\left(\alpha_{2}\right)} \sigma_{3}^{\left(\alpha_{3}\right)} \sigma_{3}^{\left(\alpha_{4}\right)} \sigma_{1}^{(A)}, \\
U \sigma_{1}^{(A)} U^{\dagger} & =\sigma_{3}^{(1)} \sigma_{3}^{(2)} \sigma_{3}^{(3)} \sigma_{3}^{(4)} \sigma_{1}^{(A)} .
\end{aligned}
$$

Note that the list contains only operators that leave the ancilla qubit $A$ unaltered. As can be seen, all single qubit terms, as well as specific two, three and four qubit interaction terms on the system qubits can be obtained. This allows one, in principle, to use Hamiltonian simulation techniques to generate any interaction Hamiltonian acting on the four system qubits. Note, however, that the generation of effective single qubit unitary operations (lines 1-3) requires in part two-body interactions. As a Hamiltonian simulation scheme operating on effective Hamiltonians may require such effective intermediate single-qubit unitaries applied for some time $t=O(1)$, the time cost to simulate certain many-body interaction Hamiltonians for time $\delta t$ will be of order $t$, i.e. a dilation factor of $t / \delta t$.

In a similar way, two $d$-level systems of this kind can be simulated and coupled pairwise. Consider in addition to qubits $1-4, A$ corresponding to a first $d=16$ dimensional system a second set of five qubits $1^{\prime}-4^{\prime}, A^{\prime}$ corresponding to a second $d=16$-dimensional system. We consider for system $1^{\prime}-4^{\prime}, A^{\prime}$ a similar graph state encoding, i.e. the the total graph has additional edges $\left(k^{\prime}, A^{\prime}\right), k^{\prime}=1^{\prime}, 2^{\prime}, 3^{\prime}, 4^{\prime}$ and $U$ changes accordingly. Again, qubit $A^{\prime}$ serves as auxiliary system and is prepared in state $|0\rangle_{x}$, and manipulation of the second $d-$ level system works in exactly the same way as for system 1 (qubits 1-4). In addition, coupling between the two $d$-level systems can e.g. be achieved via the following two-body interactions

$$
\begin{aligned}
U \sigma_{3}^{\left(\alpha_{1}\right)} \sigma_{3}^{\left(\alpha_{2}^{\prime}\right)} U^{\dagger} & =\sigma_{3}^{\left(\alpha_{1}\right)} \sigma_{3}^{\left(\alpha_{2}^{\prime}\right)} \\
U \sigma_{3}^{\left(\alpha_{1}\right)} \sigma_{1}^{\left(A^{\prime}\right)} U^{\dagger} & =\sigma_{3}^{\left(\alpha_{1}\right)}\left(\otimes_{\alpha^{\prime}=1}^{4} \sigma_{3}^{\left(\alpha^{\prime}\right)}\right) \sigma_{1}^{\left(A^{\prime}\right)} \\
U \sigma_{1}^{(A)} \sigma_{3}^{\left(\alpha_{2}^{\prime}\right)} U^{\dagger} & =\left(\otimes_{\alpha=1}^{4} \sigma_{3}^{(\alpha)}\right) \sigma_{3}^{\left(\alpha_{2}^{\prime}\right)} \sigma_{1}^{(A)} \\
U \sigma_{1}^{(A)} \sigma_{1}^{\left(A^{\prime}\right)} U^{\dagger} & =\left(\otimes_{\alpha=1}^{4} \sigma_{3}^{(\alpha)}\right)\left(\otimes_{\alpha^{\prime}=1^{\prime}}^{4^{\prime}} \sigma_{3}^{\left(\alpha^{\prime}\right)}\right) \sigma_{1}^{(A)} \sigma_{1}^{\left(A^{\prime}\right)} .
\end{aligned}
$$

The generalization to $N d$-dimensional systems that interact pairwise is straightforward. 


\section{Example 3: 2D setup with 4-body plaquette interaction}

We consider now the simulation of a Hamiltonian that contains four-body terms and corresponds to a 2D-setup where qubits are arranged on a square lattice. In particular, we consider interactions between qubits on the same plaquette $\square$, i.e. a Hamiltonian of the form

$$
H^{\prime}=\sum_{\alpha} \sigma_{3}^{(\alpha, \alpha)} \sigma_{3}^{(\alpha, \alpha+1)} \sigma_{3}^{(\alpha+1, \alpha)} \sigma_{3}^{(\alpha+1, \alpha+1)} .
$$

Such a Hamiltonian can be generated by considering $N$ qubits arranged on a rectangular array, and $N$ additional auxiliary qubits that are placed in the center of each square $\square$ and prepared in the eigenstate of $\sigma_{x},|0\rangle_{x}$. The graph is such that each of the auxiliary qubits $A_{\alpha}$ is connected to all system qubits in the corresponding square, i.e. to qubits $(\alpha, \alpha),(\alpha, \alpha+1),(\alpha+1, \alpha),(\alpha+1, \alpha+1)$. Hence, single-qubit operations on auxiliary qubit $A_{\alpha}$ transforms to a four-qubit operation on the plaquette,

$$
U \sigma_{1}^{\left(A_{\alpha}\right)} U^{\dagger}=\sigma_{3}^{(\alpha, \alpha)} \sigma_{3}^{(\alpha, \alpha+1)} \sigma_{3}^{(\alpha+1, \alpha)} \sigma_{3}^{(\alpha+1, \alpha+1)} \sigma_{1}^{A_{\alpha}},
$$

while leaving the auxiliary qubit unchanged. The simple Hamiltonian $H=B \sum_{\alpha} \sigma_{1}^{\left(A_{\alpha}\right)}$, which may e.g. be generated by applying a homogenous magnetic field in $X$-direction, hence transforms to the desired Hamiltonian $H^{\prime}$ (46). The construction can be easily generalized to higher dimensions (cubic lattice) and other geometries (e.g. hexagonal lattices).

\section{E. Time cost and universal quantum simulation}

\section{Fixed graph state encoding:}

The examples considered so far assume that the encoding (or basis change) is fixed throughout the whole simulation process. This implies that the time cost to simulate a many-body Hamiltonian $H=\sum_{k} \lambda_{k} H_{k}$ for time $t_{\text {tot }}$, where each of the terms $H_{k}$ can be obtained by a unitary conjugated single- or two-body Hamiltonian is given by

$$
\pi / 2+t_{\mathrm{tot}} \sum_{k} \lambda_{k}
$$

Here, we assume that the Hamiltonians $H_{k}$ can be obtained directly via unitary conjugation (as in example 1). That is, one has a constant overhead in simulation time, as the basis changes that need to be applied at the beginning and the end of the simulation process each have time cost $\pi / 4$ (phase gates that can be applied in parallel). There is no additional time overhead to simulate many-body Hamiltonians, as the basic single- and two-body Hamiltonians are directly transformed to the required form. If the method is only used to generate a basic $m$-body Hamiltonian for time $\delta t$, then the time cost is essentially $\pi /(2 \delta t)$, i.e. essentially the same as in the commutator method (Sec. III). However, here the implementation of the many-body Hamiltonian is exact, and the choice of time $\delta t_{m}$, the evolution time of the Hamiltonian, is not limited by requirements on the desired accuracy or the validity of approximations.

If, as discussed in example 2, the generation of the effective many-body Hamiltonian also makes use of Hamiltonian simulation techniques -in particular effective single-body unitary operations generated by twobody interactions-, one encounters an additional increase of time cost. Here, however, the graph state encoding remains fixed throughout the simulation process.

\section{Variable graph state encoding}

Another possibility is to vary the graph state encoding. Assume we wish to generate an interaction Hamiltonian

$$
H=\sum_{k} \lambda_{k} H_{k}
$$

for time short time $\delta t$, where $H_{k}$ are interaction Hamiltonians of different kind, e.g. various $m$-body terms with different $m$. While a particular graph state encoding is specially suitable to implement a specific Hamiltonian $H_{k}$ - and in fact such a graph state encoding can be chosen in a constructive way-, it might be complicated (or impossible) to generate all desired terms using the same encoding. However, one may change the encoding during the simulation process. That is, generate $e^{-i \delta t \sum_{k} \lambda_{k} H_{k}}$ via a sequence of different unitary conjugations and simple single- and two-body Hamiltonians $\tilde{H}_{k}$, i.e.

$$
\prod_{k} U_{k} e^{-i \delta t \lambda_{k} \tilde{H}_{k}} U_{k}^{\dagger} \approx e^{-i \delta t \sum_{k} \lambda_{k} U_{k} \tilde{H}_{k} U_{k}^{\dagger}}+O\left(\delta t^{2}\right) .
$$

This is similar to standard Hamiltonian simulation, except that here the intermediate unitary operations $U_{k}$ are not local, but correspond to some graph-state encoding.

Note that in such a set-up, it is even sufficient to restrict to local Hamiltonians $\tilde{H}_{k}$, which are transformed via different graph-state encodings (generated by twobody interaction) to the desired many-body interaction Hamiltonian. That is, we assume that two-body interactions between any pair of qubits and local control operations are available. In this case, the proof of universality is very easy. In fact, it is sufficient to show that a proper choice of graph state encoding allows one to generate a $m$-body interaction term

$$
\otimes_{\alpha \in S} \sigma_{j_{\alpha}}^{(\alpha)}
$$

on any subset $S$ of parties, where $j_{\alpha} \in\{1,2,3\}$. Such a basic term can be manipulated by means of standard Hamiltonian simulation techniques (i.e. intermediate local unitaries, which can be generated from single qubit (i.e. local) Hamiltonians and a graph state encoding corresponding to the trivial, empty graph, i.e. $U_{k}=\mathbb{1}$ ) to 
generate effectively any Hamiltonian acting on the subset of $S$ of qubits for some (small) time $\delta t$. As the subset $S$ is arbitrary, the same method allows one to generate arbitrary Hamiltonians, containing terms acting on different subsets $S$. If a Hamiltonian consists of $L$ basic terms of the form (51) with coefficients $\lambda_{k}$, then the time cost to simulate such a Hamiltonian is given by $L \pi / 2+\sum \lambda_{k} \delta t$. A term of the form (51) is most easily generated by choosing a graph state encoding corresponding a graph with edges $\left(\alpha, \alpha_{j}\right)$, where $\alpha$ is some fixed qubit $\in S$, and $\alpha_{j}$ runs over all remaining qubits in $S$ (i.e. qubit $\alpha$ is connected with all other qubits in $S$ ). The corresponding unitary $U$ transforms single-qubit terms acting on qubit $\alpha$ into an interaction term involving all qubits in $S$,

$$
U \sigma_{1}^{(\alpha)} U^{\dagger}=\sigma_{1}^{(\alpha)} \otimes_{\alpha_{j} \in S, \alpha_{j} \neq \alpha} \sigma_{3}^{\left(\alpha_{j}\right)}
$$

which can be transformed via local unitary operations to the desired form (51).

The method used in this proof of principle demonstration is not

optimal, as a new graph state encoding is required for each individual term. As already demonstrated in two examples above, a clever choice of graph state encoding allows one to generate many terms using the same encoding, and hence a significant reduction of the time cost can be achieved. In particular, if the Hamiltonian corresponds to some translational invariant set-up or exhibits symmetries, a proper choice of graph state encoding -where the graph has the same symmetry propertiesallows in many cases to generate all terms of the same form (connected e.g. by the translation operator) simultaneously. For instance, in example 3, two different graph state encodings, corresponding to the graph discussed in example 3 and an empty graph, are sufficient to generate an interaction Hamiltonian with arbitrary 4-qubit interaction terms. This allows, for example, to simulate a ring exchange Hamiltonian [41, 42]

$$
\left.H=\sum_{\square}\left(b_{1}^{\dagger} b_{2} b_{3}^{\dagger} b_{4}+b_{1} b_{2}^{\dagger} b_{3} b_{4}^{\dagger}\right)\right),
$$

where the sum runs over all plaquettes $\square$ and opposite corners are labelled as $1,3[2,4]$ respectively. Note that generalizations to other geometries or higher dimensions are straightforward.

\section{TELEPORTATION BASED METHODS}

A third method to generate many-body interaction terms is by using a teleportation based approach. That is, entangled states are used as a resource to generate by means of teleportation an appropriate multi-qubit unitary operation, corresponding to a time evolution with respect to a certain many-body Hamiltonian $H$. In the following we will discuss two teleportation based methods that allow one to generate time evolutions with respect to a Hamiltonian of the form

$$
H=\otimes_{\alpha \in S} \sigma_{3}^{(\alpha)},
$$

for an arbitrary time $t$ in a deterministic way, where $S$ is some set of $|S|$ qubits. We will consider $|S|=m$ in the following. This corresponds to the implementation of a unitary operation of the form $U_{\varphi} \equiv \exp (-i \varphi H)$ with $H$ given by Eq. 54 and $\varphi=t$.

Different methods to generate specific, non-local unitary operations are known. Except in special cases (e.g. for a CNOT gate), either a sequence of operations using a set of entangled states, or entangled states of higher dimension (including some auxiliary particles) are required to make the process deterministic. There exist a one to one correspondence between arbitrary quantum states $E$ (described by a density operator) and completely positive maps $\mathcal{E}$ [43, 44, 45]. The state $E$ (possibly shared between many parties) can be used as a resource to implement by means of local operations and classical communication (essentially a teleportation process) the map $\mathcal{E}$ on an arbitrary input state probabilistically (i.e. for certain measurement outcomes in the teleportation process). In many relevant cases, e.g. for gates of the form $U_{\varphi}$, either $U_{\varphi}$ or $U_{\varphi}^{\dagger}$ is implemented with probability $p=1 / 2$. In this case, the implementation of $U_{\varphi}$ can be made deterministic by using a sequence of teleportation processes [44] corresponding to unitary operations $U_{\varphi}, U_{2 \varphi}, U_{4 \varphi}$, etc., where additional unitaries are only applied if the previous unitary was not implemented successfully. Alternatively, a different entangled state consisting of additional auxiliary particles can be used for teleportation, where the measurement basis of auxiliary particles are determined by the measurement outcomes of the teleportation process. In [46, 47] such a method is demonstrated for the purposes simulating Hamiltonians that are diagonal in the computational basis. This approach is similar to the measurement based one-way quantum computation using cluster states [48]. We will discuss both methods in detail in the following.

Cirac et al [44] demonstrated that the Jamiolkowski isomorphism defines a teleportation protocol that allows us to use entangled states as a resource for generating entangling unitary evolutions. In this section we revisit their work and demonstrate several related protocols that allow us to use entangled states to generate entangling unitary operations.

\section{A. The Jamiołkowski Isomorphism}

We now review some of the features of the Jamiołkowski isomorphism. This review is incomplete and discusses only those features of the isomorphism that we feel are necessary to understand the teleportation protocols which will be discussed later in this section. For a more complete treatment we refer the reader to [49, 50].

The Jamiołkowski isomorphism is a mapping between the set of linear operators and the matrix algebra defined 
on a composite Hilbert space. More precisely, given the composite Hilbert space $\mathcal{H}_{A^{\prime}} \otimes \mathcal{H}_{A}$ and a matrix $E$ defined on this composite space then the isomorphism is given by

$$
\mathcal{E}(M)=d_{A}^{2} \operatorname{tr}_{A \bar{A}}\left[E^{A^{\prime} A} P_{\Phi}^{A \bar{A}} M^{\bar{A}}\right]
$$

where $H^{\bar{A}}$ has the same dimension as $H^{A}, M$ is any matrix defined in the matrix algebra of $H^{A}$ (and subsequently is also defined for $H^{\bar{A}}$ ), and $P_{\Phi}^{A \bar{A}}$ is a projector representing the maximally entangled state

$$
|\Phi\rangle=\frac{1}{\sqrt{d_{A}}} \sum_{i=1}^{d_{A}}|i\rangle^{\bar{A}}|i\rangle^{A}
$$

where $d_{A}$ is the dimension of system $A$, and denote $P_{\Phi}=$ $|\Phi\rangle\langle\Phi|$. The inverse mapping is given by

$$
E=\mathcal{E}^{\bar{A}} \otimes \mathcal{I}\left(P_{\Phi}^{\bar{A} A}\right)
$$

where $\mathcal{I}$ is the identity map.

It should be clearly noted that the Jamiołkowski isomorphism is defined for the set of all linear operators and the set of all matrices for the composite Hilbert space $\mathcal{H}_{\bar{A}} \otimes \mathcal{H}_{A}$. This set of physical maps is a subset of this set of linear operators. Likewise, the set of quantum density matrices is a subset of all matrices on this composite Hilbert space. It turns out, that we can define a teleportation-like protocol that helps us to understand the circumstances for which the isomorphism is physically relevant.

If we have a maximally entangled state, $|\Phi\rangle^{\bar{A} A}$, and we act with a trace-preserving completely positive map, $\mathcal{E} \otimes \mathcal{I}$, on the system then clearly the resulting state, $E$ is a quantum state. See figure 1. We shall now see how the state $E$ can be used to probabilistically teleport the operation $\mathcal{E}(\rho)$ onto some arbitrary state $\rho$. Consider the composite system $A^{\prime} A \bar{A}$ with Hilbert space $\mathcal{H}_{A^{\prime}} \otimes \mathcal{H}_{A} \otimes$ $\mathcal{H}_{\bar{A}}$. Prepare $E$ on the composite system $A^{\prime} A$ and system $\bar{A}$ is in some arbitrary state, $\rho$,

$$
\rho_{A^{\prime} A \bar{A}}=E \otimes \rho .
$$

Then, if we then perform a projective measurement on $\rho_{A^{\prime} A \bar{A}}$ in a maximally entangled basis which includes $|\Phi\rangle_{A \bar{A}}$, the Jamiołkowski isomorphism tells us directly that there is a $\frac{1}{d_{A}^{2}}$ probability that the outcome of this measurement will leave system $A^{\prime}$ in the state $\mathcal{E}(\rho)$. Thus, we say that with probability $\frac{1}{d_{A}^{2}}$, the operation $\mathcal{E}(\rho)$ is "teleported to system $A^{\prime \prime}$ ". Such a process is outlined in figure 2 .

\section{The Jamiotkowski isomorphism for many-body systems}

We now turn to the case when $A$ is a composite system. Following the description in [49? ] we will consider the case where $A$ and $A^{\prime}$ are many-body systems that have Hilbert spaces of the same type. That is we can write,

$$
\mathcal{H}^{A}=\mathcal{H}^{A_{1}} \otimes \ldots \otimes \mathcal{H}^{A_{N}},
$$

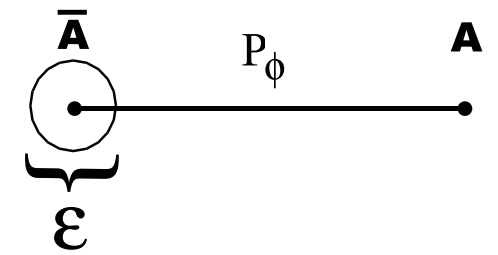

FIG. 1: In order to obtain the state $E$ the completely positive map $(\mathrm{CPM}) \mathcal{E}$ is applied to system $\bar{A}$ of the joint system of $A$ and $\bar{A}$, which is prepared in the maximally entangled state $P_{\Phi}^{\bar{A} A}$. (Figure taken from Ref. [49])

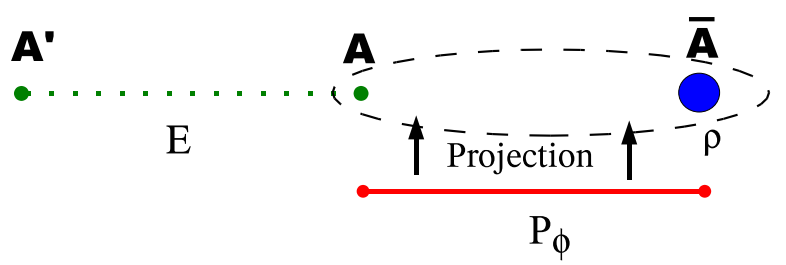

FIG. 2: (Color online) Given the state $E$ on the composite system $A^{\prime}$ and $A$, the CPM $\mathcal{E}$ is evaluated for an arbitrary input state $\rho$ by taking $\rho$ as an input at system $\bar{A}$. Then the joint system $A \bar{A}$ is measured in a Bell basis containing the maximally entangled state $P_{\Phi}^{A \bar{A}}$. With probability $\frac{1}{d_{A}^{2}}$ the desired output state $\mathcal{E}(\rho)$ is then obtained at system $A^{\prime}$. (Figure taken from Ref. [49])

where each constituent Hilbert-space has dimension $d_{A_{i}}$ and $\mathcal{H}^{A} \simeq \mathcal{H}^{A^{\prime}}$. If we choose the projector $P_{\Phi}^{\bar{A} A}$ to be the tensor product of projectors onto the two-qudit maximally entangled states $|\Phi\rangle^{\bar{A}_{i} A_{i}}$ then we can have a straightforward way of extending the isomporphism to the many-body setting and retain the interpretation in terms of a teleportation protocol [50]. More precisely $P_{\Phi}^{\bar{A} A}$ is given by,

$$
P_{\Phi}^{\bar{A} A}=P_{\Phi}^{\bar{A}_{1} A_{1}} \otimes \ldots \otimes P_{\Phi}^{\bar{A}_{N} A_{N}}
$$

where

$$
|\Phi\rangle=\frac{1}{\sqrt{d_{A_{i}}}} \sum_{k=1}^{d_{A_{i}}}|k\rangle^{\bar{A}_{i}}|k\rangle^{A_{i}} .
$$

The only difference between teleportation protocols in the many-body case and the case that we have already discussed is that in this many-body scenario, the teleportation protocol requires $N$ projective measurements to be made between the systems $A$ and $\bar{A}$ instead of one. The definition of $E$ in the many-body case is depicted in figure 3. The many-body teleportation protocol for the implementation of $\mathcal{E}(\rho)$ on system $A^{\prime}$ demonstrated in figure 4. 


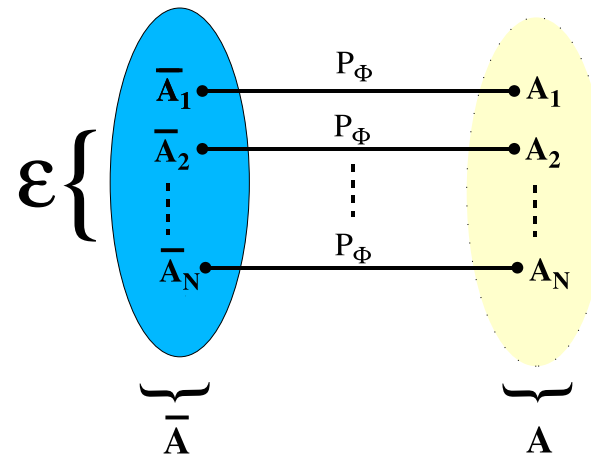

FIG. 3: (Color online) In order to obtain the state $E$ the CPM $\mathcal{E}$ is applied to the subsystems $\bar{A}_{i}$ of the composite system $\bar{A}=$ $\left(\bar{A}_{1}, \ldots, \bar{A}_{N}\right)$, which are (locally) prepared in the maximally entangled states $P_{\Phi}^{\bar{A}_{i} A_{i}}$. (Figure taken from Ref. [49])

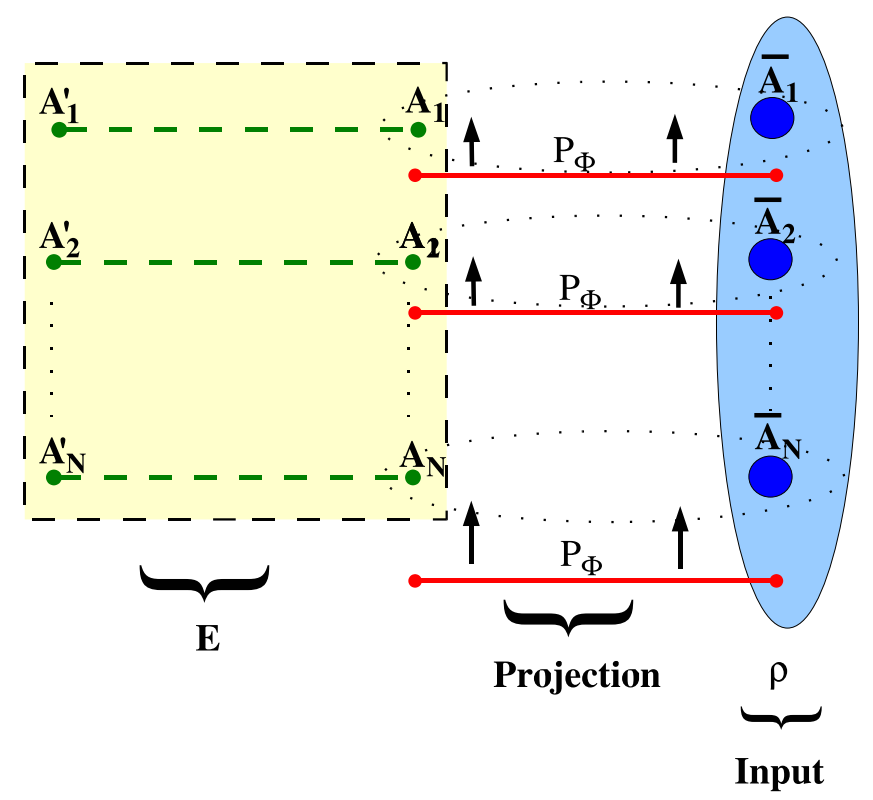

FIG. 4: (Color online) Given the state $E$ on the composite system $A^{\prime}=\left(A_{1}^{\prime}, \ldots, A_{N}^{\prime}\right)$ and $A=\left(A_{1}, \ldots, A_{N}\right)$, the CPM $\mathcal{E}$ is evaluated for an arbitrary multipartite input state $\rho$ by taking $\rho$ as an input at system $\bar{A}=\left(\bar{A}_{1}, \ldots, \bar{A}_{N}\right)$. Then the joint systems $A_{i} \bar{A}_{i}$ are (locally) measured in a Bell basis containing the maximally entangled state $P_{\Phi}^{A_{i} \bar{A}_{i}}$. With probability $\frac{1}{d_{A}^{2}}$ the desired output state $\mathcal{E}(\rho)$ is then obtained at system $A^{\prime}$. (Figure taken from Ref. [49])

\section{B. Teleportation-based gates using weakly entangled states}

As we have seen, the Jamiołkowski isomorphism shows us how to create a teleportation protocol that probabilistically generates quantum operations when given certain entangled quantum states. For the purposes of this paper we are interested in simulation protocols that generate unitary operations of the form $U(\alpha)=e^{-i \alpha \sigma_{z}^{\otimes n}}$. Such a protocol was described in [44] where the au- thors demonstrated a non-deterministic simulation protocol based on the Jamiołkowski isomorphism that succeeds with a higher success probability than the protocol that we discussed in the previous subsection. In the next section we will demonstrate how this protocol can be made deterministic through the use of an additional ancillary system.

\section{Quantum control and the Jamiotkowski isomorphism}

In order to understand the protocol demonstrated in [44], it helps to have an understanding of the way in which control operations on a state, $E$, are transferred to the associated operation $\mathcal{E}$ under the Jamiołkowski isomorphism. A list of control rules is given in [49], here we primarily interested in two of these rules:

1. Given matrices $B_{1}, C_{1}$ that can be enacted on system $A^{\prime}$ and $B_{2}, C_{2}$ on system $A$, then the matrix,

$$
E^{\prime}=B_{1}^{A^{\prime}} \otimes B_{2}^{A} E^{\mathcal{E}} C_{1}^{A^{\prime}} \otimes C_{2}^{A}
$$

is isomorphic to the map,

$$
\mathcal{E}^{\prime}(M)=B_{1} \mathcal{E}\left(B_{2}^{T} M C_{2}^{T}\right) C_{1}
$$

where the superscript $T$ denotes transposition.

2. $\mathcal{E}$ is separable with respect to $A_{k}$ and $A_{l}$ iff $E$ is separable with respect to $A_{k}$ and $A_{l}$. That is the CPM corresponding to state $E \otimes F$ is $\mathcal{E} \otimes \mathcal{F}$, where $\mathcal{E}$ and $\mathcal{F}$ are the maps isomorphic to $E$ and $F$.

The first rule can be extended to demonstrate that if control is locally limited to parties $A$ and $A^{\prime}$ then the allowable set of control operations are limited to local operations with one-way classical communication. The only allowable classical communication must flow from part $A$ to party $A^{\prime}$ as the manipulations performed by party $A$ occur prior to implementing the map $\mathcal{E}$ whereas those performed by $A$ occur after. To see this, consider the following mixture of matrices,

$$
E^{\prime}=\sum_{j} Q_{j}^{A^{\prime}} \otimes R_{j}^{A} E\left(Q_{j}^{A^{\prime}} \otimes R_{j}^{A}\right)^{\dagger} .
$$

Using Eq. 62 we see that the corresponding CPM is,

$$
\mathcal{E}^{\prime}=\sum_{j} Q_{j} \mathcal{E}\left(R_{j}^{T} M R_{j}^{*}\right) Q_{j}^{\dagger} .
$$

If the matrices $\left\{Q_{j}\right\}$ and $\left\{R_{j}\right\}$ represent measurements and unitary operations, then the operation $R_{j}$ occurs before the operation $Q_{j}$. Thus the set of operations that can physically be performed on the state $E$ are restricted to,

$$
E^{\prime}=\sum_{i j} Q_{i j}^{A^{\prime}} \otimes R_{j}^{A} E\left(Q_{i j}^{A^{\prime}} \otimes R_{j}^{A}\right)^{\dagger}
$$


where the $\mathrm{CPM} \mathcal{R} \rho=\sum_{j} R_{j} \rho R_{j}^{\dagger}$ is bi-stochastic tracepreserving $\mathrm{CPM}^{1}$, and if $j$ corresponds to a measurement outcome at party $A$ the operation $Q_{j}(\rho)=\sum_{i} B_{i j} \rho B_{i j}^{\dagger}$ is a trace-preserving CPM. It is interesting to note that in the case where all operations are either unitary or projective measurements then the bi-stochasticity of $\mathcal{R}$ is assured. This can be seen by noting that for any unitary operator $U,\left(U^{*}\right)^{\dagger}=U^{T}=\left(U^{*}\right)^{-1}$ and all projectors onto pure states are real and Hermitian.

\section{Using weakly entangled states to generate phase gates}

In this section we review the non-deterministic simulation protocol for generating unitary operations of the form $U(\alpha)=e^{-i \alpha \sigma_{z}^{\otimes n}}$ that was presented in [50].

Firstly, we shall give the two-qubit protocol that appeared in [4] in which it was shown how to generate the unitary operator, $e^{-i \alpha \sigma_{z} \otimes \sigma_{z}}$, on any given input state. We will then discuss why this protocol works and how to extend it to the more general many-qubit case.

1. Prepare the state,

$$
\begin{aligned}
|\alpha\rangle= & \cos \alpha\left|\Phi^{+}\right\rangle^{A_{1} A_{1}^{\prime}}\left|\Phi^{+}\right\rangle^{A_{2} A_{2}^{\prime}} \\
& -i \sin \alpha\left|\Phi^{-}\right\rangle^{A_{1} A_{1}^{\prime}}\left|\Phi^{-}\right\rangle^{A_{2} A_{2}^{\prime}}
\end{aligned}
$$

where $\alpha=\pi / 2^{N},\left|\Phi^{+}\right\rangle=1 / \sqrt{2}(|00\rangle+|11\rangle)$ and $\left|\Phi^{-}\right\rangle=1 / \sqrt{2}(|00\rangle-|11\rangle)$. The state, $|\alpha\rangle$, is prepared between parties $A$ and $A^{\prime}$.

2. Introduce party $\bar{A}$ which is in the desired input state $\rho$.

3. Perform a Bell basis measurements between $A_{1} \bar{A}_{1}$ and $A_{2} \bar{A}_{2}$. Note that we can write the Bell basis as

$$
\left|\Psi_{i_{1}, i_{2}}\right\rangle=\mathbb{1} \otimes \sigma_{i_{1}, i_{2}}\left|\Phi^{+}\right\rangle,
$$

where $\sigma_{1,1}=\mathbb{1}, \sigma_{1,2}=\sigma_{z}, \sigma_{2,1}=\sigma_{y}$, and $\sigma_{2,2}=$ $\sigma_{x}$.

4. If the measurement on $A_{1} \bar{A}_{1}$ produces the outcome $\left|\Psi_{i_{1}, i_{2}}\right\rangle$, apply $\sigma_{i_{1}, i_{2}}$ to qubit $A_{1}^{\prime}$. If the measurement on $A_{2} \bar{A}_{2}$ produces the outcome $\left|\Psi_{j_{1}, j_{2}}\right\rangle$, apply $\sigma_{j_{1}, j_{2}}$ to qubit $A_{2}^{\prime}$.

5. If $i_{1}=j_{1}$ then $U(\alpha)=e^{-i \alpha \sigma_{z} \otimes \sigma_{z}}$ has been applied to system $A^{\prime}$ and the procedure terminates. This occurs with probability $1 / 2$.

6. If $i_{1} \neq j_{1}$ then we have applied $U(-\alpha)$ to system $A^{\prime}$. This occurs with probability $1 / 2$. In this case, we re-label system $A^{\prime}$ as system $\bar{A}$ and then prepare the state $|2 \alpha\rangle$ on $A A^{\prime}$ and repeat the procedure.

\footnotetext{
1 Thus the map $\tilde{\mathcal{R}}=\sum_{j} R_{j}^{T} \rho R_{j}^{*}$ is also a trace-preserving CPM
}

The probability of success, that is that $U(\alpha)$ has been applied to system $A^{\prime}$, upon repetition is $1 / 2$. With probability $1 / 2$ the unitary $U(-3 \alpha)$ is applied to system $A^{\prime}$.

7. For the $k$ th iteration we prepare the state $\left|2^{k-1} \alpha\right\rangle$. After $N$ steps the procedure must necessarily terminate as $U\left(-\left(2^{N}-1\right) \alpha\right)=U(\alpha)$ up to an irrelevant global phase.

Why does this procedure work? It is a variation of the teleportation protocol that we saw in the previous section. The state $|\alpha\rangle$ can be prepared by the following operation on the pair maximally entangled states $\left|\Phi^{+}\right\rangle^{A_{1} A_{1}^{\prime}}\left|\Phi^{+}\right\rangle^{A_{2} A_{2}^{\prime}}$

$$
|\alpha\rangle=e^{-i \alpha \mathbb{1} \otimes \sigma_{z} \otimes \mathbb{1} \otimes \sigma_{z}}\left|\Phi^{+}\right\rangle^{A_{1} A_{1}^{\prime}}\left|\Phi^{+}\right\rangle^{A_{2} A_{2}^{\prime}} .
$$

Looking at the definition of the the Jamiolkowski isomorphism and noting that $A, A^{\prime}$, and $\bar{A}$ are each qubits then it is clear that the state $|\alpha\rangle\langle\alpha|=E$ and it's corresponding $\mathrm{CPM}$ is $\mathcal{E} \equiv U(\alpha)$. According to the teleportation protocol of the previous section, in order to apply $U(\alpha)$ to an unknown state we need to perform Bell-basis measurements on systems $A$ and $\bar{A}$. For the state $|\alpha\rangle$ given above, a successful run of the protocol coincides with measuring systems $A_{1} \bar{A}_{1}$ and $A_{2} \bar{A}_{2}$ to be in the state $\left|\Phi^{+}\right\rangle$, this occurs with probability $1 / 16$.

In the protocol that we have presented in this section, we use local unitary operations and one-way classical communication to increase this success probability to $1 / 2$ for any given instance of the protocol. The key to this improved success probability is that the Pauli correction operators we apply, $\sigma_{i_{1}, i_{2}}$ and $\sigma_{j_{1}, j_{2}}$, allow us to manipulate the state $\alpha$.

A Bell basis can be generated by applying a singlequbit Pauli operator to the state $\left|\Phi^{+}\right\rangle$,

$$
\left|\Psi_{i_{1}, i_{2}}\right\rangle=\mathbb{1} \otimes \sigma_{i_{1}, i_{2}}\left|\Phi^{+}\right\rangle .
$$

This equation tells us that performing a measurement in this basis and getting the outcome $\left|\Psi_{i_{1}, i_{2}}\right\rangle$ is is equivalent to applying the projector $\left|\Phi^{+}\right\rangle$followed by the Pauli operation $\sigma_{i_{1}, i_{2}}$. Interestingly, it is irrelevant which qubit the operation $\sigma_{i_{1}, i_{2}}$ is applied to. In Step 3 of this protocol we perform Bell measurements between systems $A$ and $\bar{A}$. If the outcome of these measurements are all $\left|\Phi^{+}\right\rangle$ we have directly applied the gate $U(\alpha)$. Alternatively, we can see from Equations (62) and (65) that we will have applied the map $\mathcal{E}\left(\left(\sigma_{i_{1}, i_{2}} \otimes \sigma_{j_{1}, j_{2}}\right) \rho\left(\sigma_{i_{1}, i_{2}} \otimes \sigma_{j_{1}, j_{2}}\right)\right)$. In the case where $i_{1}=j_{1}$ we can apply the corrections of given in Step 4 of the protocol above and succeed in applying $U(\alpha)$. These corrections work because they commute with the unitary $U(\alpha)$. In the case where $i_{1} \neq j_{1}$, the effect of applying the correction is to apply $U(-\alpha)$ to $\rho$, as

$$
U(\alpha) \sigma_{i_{1}, i_{2}} \otimes \sigma_{j_{1}, j_{2}}=\sigma_{i_{1}, i_{2}} \otimes \sigma_{j_{1}, j_{2}} U(-\alpha) .
$$

This protocol works half of the time because $U(\alpha)$ commutes with half of the set of two-qubit Pauli product 
operators $\left\{\sigma_{j} \otimes \sigma_{k} \mid j, k=0, \ldots, 3\right\}$. In the next section we demonstrate how to make a similar protocol that deterministically applies $U(\alpha)$ to $\rho$ through the use of an ancillary qubit.

As has been shown in [50], one can generalize this protocol to produce evolutions by the $n$-qubit unitary $U(\alpha)=e^{-i \alpha \sigma_{z}^{\otimes n}}$. In this case the probability of a success in any instance of the protocol is still $1 / 2$ as in the twoqubit case. In essence the protocol is the same. Prepare the state $|\alpha\rangle$ using $n$ Bell pairs, perform $n$ Bell-basis measurements, apply $n$ Pauli correction operations. In each instance the correction operations are the same as those for the two-qubit case. That is, if $\left|\Psi_{i_{1}, i_{2}}\right\rangle$ is measured, apply the correction $\sigma_{i_{1}, i_{2}}$ on the appropriate qubit. Like in the two-qubit case, success is in an instance of the protocol is achieved in the case when tensor product of all of the Pauli correction operations that are applied commutes with the operator $\sigma_{z}^{\otimes n}$, which occurs with a success probability of $\frac{1}{2}$.

\section{Teleportation-based gates using GHZ-type states}

In the previous section we saw how we can use teleportation as a primitive to stochastically generate unitary operations of the form $U=e^{-i \alpha \sigma_{z}^{\otimes n}}$. It has been demonstrated [51, 52] that gate-teleportation protocols like this are always deterministic if the entangling operation to be teleported onto $\rho$ is an element of the Clifford group (defined as the normalizer of the Pauli group). If an operation is not in this group, as is the case with $U(\alpha)$, then the necessary correction operations in a deterministic protocol are entangling.

In this section we shall demonstrate that by performing teleportation using GHZ-type states as a primitive we can deterministically "teleport" the non-Clifford operations like $U(\alpha)$. In essence, by utilizing prior entanglement with ancillary qubits we can avoid applying entangling correction operations to the teleportation protocol. The simulation protocol that we demonstrate here is essentially the same as those which was demonstrated in the context of graph-state based simulation in [46, 47]. However, here we use the language of teleportation in order to highlight the connection with the work presented in [44, 50].

Consider the state

$$
|G H Z\rangle=\frac{1}{\sqrt{2}}\left(|+\rangle^{\otimes n}|0\rangle_{E}+|-\rangle^{\otimes n}|1\rangle_{E}\right),
$$

this state is an $n+1$ qubit GHZ-state. The GHZ state is a two-colorable graph state. It is easy to see that this state can be generated from phase gates by applying a phase gate between qubit $E$ and each of the other $n$ qubits in the system. It has recently been shown that such states can be purified [53, 54, 55, 56].

\section{Simulation protocol}

In Section IV B 2 we saw a teleportation protocol that used weakly entangled states as a resource to generate $U(\alpha)$ that had a probability of success in any given iteration of $1 / 2$. This protocol failed when the Pauli correction operations did not commute with $U(\alpha)$. When this occurred, the result of the protocol was to apply the operator $U(-\alpha)$ to the state $\rho$ because the correction operation commutes through $U(\alpha)$ to generate $U(-\alpha)$. Interestingly, in a failing instance of the protocol, if we could "go back in time" and change the weakly entangled state $|\alpha\rangle$ to $|-\alpha\rangle$ we would have applied the operator $U(\alpha)$ to $\rho$.

In this section we demonstrate an alternate method for generating the states $|\alpha\rangle$ or $|-\alpha\rangle$, that is the states corresponding to the maps $U=e^{-i \alpha \sigma_{z} \otimes \sigma_{z}}$ and $U=e^{i \alpha \sigma_{z} \otimes \sigma_{z}}$. This method allows us to generate these states after performing Bell measurements between systems $\bar{A}$ and $A$.

Consider the following $G H Z$-like state,

$$
\begin{aligned}
|\kappa\rangle= & \frac{1}{\sqrt{2}}\left(\left|\Phi^{+}\right\rangle^{A_{1} A_{1}^{\prime}}\left|\Phi^{+}\right\rangle^{A_{2} A_{2}^{\prime}}|0\rangle^{E}\right. \\
& \left.+\mathbb{1} \otimes \sigma_{z} \otimes \mathbb{1} \otimes \sigma_{z}\left|\Phi^{+}\right\rangle^{A_{1} A_{1}^{\prime}}\left|\Phi^{+}\right\rangle^{A_{2} A_{2}^{\prime}}|1\rangle^{E}\right)
\end{aligned}
$$

where the superscript $E$ denotes the state of an ancillary system. We define the following measurement basis for system $E$ :

$$
\begin{aligned}
\left\{|m\rangle,\left|m_{\perp}\right\rangle\right\}= & \{\cos (\alpha)|0\rangle+i \sin (\alpha)|1\rangle, \\
& i \sin (\alpha)|0\rangle+\cos (\alpha)|1\rangle\} \\
\left\{|-m\rangle,\left|-m_{\perp}\right\rangle\right\}= & \{\cos (\alpha)|0\rangle-i \sin (\alpha)|1\rangle, \\
& i \sin (\alpha)|0\rangle-\cos (\alpha)|1\rangle\} .
\end{aligned}
$$

If we re-write the state $|\kappa\rangle$ in terms of the $\left\{|m\rangle,\left|m_{\perp}\right\rangle\right\}$ we find,

$$
\begin{aligned}
|\kappa\rangle= & \frac{1}{\sqrt{2}}\left[\left(\cos (\alpha)\left|\Phi^{+}\right\rangle^{A_{1} A_{1}^{\prime}}\left|\Phi^{+}\right\rangle^{A_{2} A_{2}^{\prime}}\right.\right. \\
& \left.-i \sin (\alpha) \mathbb{1} \otimes \sigma_{z} \otimes \mathbb{1} \otimes \sigma_{z}\left|\Phi^{+}\right\rangle^{A_{1} A_{1}^{\prime}}\left|\Phi^{+}\right\rangle^{A_{2} A_{2}^{\prime}}\right)|m\rangle^{E} \\
& +\left(\cos (\alpha) \mathbb{1} \otimes \sigma_{z} \otimes \mathbb{1} \otimes \sigma_{z}\left|\Phi^{+}\right\rangle^{A_{1} A_{1}^{\prime}}\left|\Phi^{+}\right\rangle^{A_{2} A_{2}^{\prime}}\right. \\
& \left.\left.+i \sin (\alpha)\left|\Phi^{+}\right\rangle^{A_{1} A_{1}^{\prime}}\left|\Phi^{+}\right\rangle^{A_{2} A_{2}^{\prime}}\right)\left|m_{\perp}\right\rangle^{E}\right] .
\end{aligned}
$$

Which can be simplified to,

$$
\begin{aligned}
& |\kappa\rangle=\frac{1}{\sqrt{2}}\left[e^{-i \alpha \sigma_{z}^{A_{1}^{\prime}} \sigma_{z}^{A_{2}^{\prime}}}\left|\Phi^{+}\right\rangle^{A_{1} A_{1}^{\prime}}\left|\Phi^{+}\right\rangle^{A_{2} A_{2}^{\prime}}|m\rangle^{E}\right. \\
& \left.+\sigma_{z}^{A_{1}^{\prime}} \sigma_{z}^{A_{2}^{\prime}} e^{-i \alpha \sigma_{z}^{A_{1}^{\prime}} \sigma_{z}^{A_{2}^{\prime}}}\left|\Phi^{+}\right\rangle^{A_{1} A_{1}^{\prime}}\left|\Phi^{+}\right\rangle^{A_{2} A_{2}^{\prime}}\left|m_{\perp}\right\rangle^{E}\right] \\
& =\frac{1}{\sqrt{2}}\left(|\alpha\rangle|m\rangle+\sigma_{z}^{A_{1}^{\prime}} \sigma_{z}^{A_{2}^{\prime}}|\alpha\rangle\left|m_{\perp}\right\rangle\right) \text {. }
\end{aligned}
$$

Eq. 77 demonstrates that a measurement on $|\kappa\rangle$ of system $E$ in the $\left\{|m\rangle,\left|m_{\perp}\right\rangle\right\}$ basis will either generate the state $|\alpha\rangle$, if the outcome is $m$, or the state $\sigma_{z}^{A_{1}^{\prime}} \sigma_{z}^{A_{2}^{\prime}}|\alpha\rangle$, given $m_{\perp}$ as the outcome. By performing a similar analysis it is possible to see that a measurement in the basis 
$\left\{|-m\rangle,\left|-m_{\perp}\right\rangle\right\}$ produces either the state $|-\alpha\rangle$ or the state $\sigma_{z}^{A_{1}^{\prime}} \sigma_{z}^{A_{2}^{\prime}}|-\alpha\rangle$. Given that we can always perform Pauli correction operations on system $A^{\prime}$ after this measurement, we see that a measurement on the ancilla system $E$ can generate the states $|\alpha\rangle$ or $|-\alpha\rangle$.

With this technique in mind, we shall now outline our new protocol.

We first consider the two-qubit case. We want to deterministically generate an evolution by the unitary $U=e^{-i \alpha \sigma_{z} \otimes \sigma_{z}}$ on the unknown two-qubit state $\rho$ for any $\alpha$.

1. Prepare the resource state $|\kappa\rangle$

2. Introduce party $\bar{A}$ which is in the desired input state $\rho$.

3. Perform Bell basis measurements between $A_{1} \bar{A}_{1}$ and $A_{2} \bar{A}_{2}$.

4. If the measurement on $A_{1} \bar{A}_{1}$ produces the outcome $\left|\Psi_{i_{1}, i_{2}}\right\rangle$, apply $\sigma_{i_{1}, i_{2}}$ to qubit $A_{1}^{\prime}$. If the measurement on $A_{2} \bar{A}_{2}$ produces the outcome $\left|\Psi_{j_{1}, j_{2}}\right\rangle$, apply $\sigma_{j_{1}, j_{2}}$ to qubit $A_{2}^{\prime}$.

5. If $i_{1}=j_{1}$, then measure system $E$ in the $\left\{|m\rangle,\left|m_{\perp}\right\rangle\right\}$ basis. If the outcome is $m$ the procedure terminates. If the outcome is $m_{\perp}$ then apply a $\sigma_{z}$ operation to qubits $A_{1}^{\prime}$ and $A_{2}^{\prime}$ to terminate the procedure.

6. If $i_{1} \neq j_{1}$, then measure system $E$ in the $\{|-m\rangle, \mid-$ $\left.\left.m_{\perp}\right\rangle\right\}$ basis. If the outcome is $m$ the procedure terminates. If the outcome is $-m_{\perp}$ then apply a $\sigma_{z}$ operation to qubits $A_{1}^{\prime}$ and $A_{2}^{\prime}$ to terminate the procedure.

7. Upon termination, system $A^{\prime}$ will have undergone the evolution

$$
\mathcal{E}\left(\rho^{A^{\prime}}\right)=U(\alpha) \rho U(\alpha)^{\dagger} .
$$

Essentially, the procedure that we have outlined here is the same as that for weakly entangled states. The key difference is that in the case of the protocol for weakly entangled states, we constructed $|\alpha\rangle$ first. Under that protocol, we are restricted to manipulations that are local operations with one-way classical communication. In this protocol, we keep systems $A$ and $A^{\prime}$ entangled with system $E$ until the final step. The effect of measuring system $E$ is to collapse the total composite system into the desired state, either $|\alpha\rangle$ or $|-\alpha\rangle$.

This protocol is easily extended to generate evolutions of the form $U=e^{-i \alpha \sigma_{z}^{\otimes n}}$. Firstly prepare the state

$$
\begin{aligned}
& |\kappa\rangle=\frac{1}{\sqrt{2}}\left(\left|\Phi^{+}\right\rangle^{A_{1} A_{1}^{\prime}} \ldots\left|\Phi^{+}\right\rangle^{A_{n} A_{n}^{\prime}}|0\rangle\right. \\
& \left.+\sigma_{z}^{A_{1}^{\prime}} \ldots \sigma_{z}^{A_{n}^{\prime}}\left|\Phi^{+}\right\rangle^{A_{1} A_{1}^{\prime}} \ldots\left|\Phi^{+}\right\rangle^{A_{n} A_{n}^{\prime}}|1\rangle\right) \text {. }
\end{aligned}
$$

Then perform the same Bell measurements and Pauli corrections as above. If the operations applied after the measurement in Step 4 commute with the operator $\sigma_{z}^{A_{1}^{\prime}} \ldots \sigma_{z}^{A_{n}^{\prime}}$, then measure in the $\left\{|m\rangle,\left|m_{\perp}\right\rangle\right\}$ basis and apply the operator $\sigma_{z}^{A_{1}^{\prime}} \ldots \sigma_{z}^{A_{n}^{\prime}}$ if the outcome is $m_{\perp}$. If they do not, measure in the $\left\{|-m\rangle,\left|-m_{\perp}\right\rangle\right\}$ basis and apply $\sigma_{z}^{A_{1}^{\prime}} \ldots \sigma_{z}^{A_{n}^{\prime}}$ if the outcome is $m_{\perp}$.

\section{PART II: Influence of noise}

As we discussed in the Introduction, building a faulttolerant quantum computer is a challenging task that may not be accomplished in the near future. In the meantime it is hoped that non-trivial quantum simulation experiments could still be performed. In particular, the demonstration of an imperfect quantum simulation that could still outperform the best-known simulation algorithms on a classical computer would be a significant experimental achievement.

In Part II of this paper, we discuss the influence of noise and imperfections on the simulation of many-body Hamiltonians using the different methods described in Part I. We focus our attention on protocols for simulating Hamiltonian evolutions of the form $H_{\alpha}=e^{-i \alpha \sigma_{z}^{\otimes m}}$. As we saw in Part I, while Hamiltonians such as $H_{\alpha}$ have a particularly simple form, they can be used alongside the Lie-Trotter formula to simulate the dynamics of any finite-dimensional Hamiltonian. Our focus is on these more simple Hamiltonians because in many cases the effects of noise on these protocols can be analytically studied. This gives us insight into the types of errors that might occur in more sophisticated simulation protocols. In particular, the following analysis could be used to derive error bounds on the measurement statistics of more complex simulations that use Hamiltonians like $H_{\alpha}$ as a building block.

We begin Part II by discussing general methods for classifying noise processes. We then go on to introduce the noise models that we consider, local dephasing and depolarizing noise and errors induced by random errors in the evolution time of entangling Hamiltonians. We then set about integrating these noise models into the protocols that we discussed in Part I and examining the effects analytically, where possible, and computationally. Mostly, we look at the effect of introducing noise to the entangling operations in these protocols and consider local operations to be perfect. This is done because for the purposes of quantum simulation we are concerned with the way that the noise is spread throughout the system as a result of the entangling operations in each protocol. In addition, we often focus on regimes where the effect of local noise on single-qubit operations can be incorporated into the noise model for the entangling operations without much loss of generality. 


\section{DISTANCE MEASURES AND FIDELITY}

We will use two different measures to quantify the quality of the simulation process. Time evolution with respect to a specific many-body Hamiltonian $H$ for a time $t$ corresponds to a unitary operation $U_{t}$ (which is the desired evolution), while time evolution governed by a master equation of Lindblad form (describing the noisy evolution), leads to a completely positive map $\mathcal{E}_{t}$. We consider the case where the operation is acting on $m$ qubits and determine how close $\mathcal{E}_{t}$ is to $U_{t}$.

We use two devices for comparing the closeness of $\mathcal{E}_{t}$ to $U_{t}$, Jamiołkowski fidelity [58] and the local noise equivalent. The mathematical detail of both of these devices are discussed in more detail below. It is worth noting here that both the Jamiołkowski fidelity and the local noise equivalent have useful operational interpretations when being used to describe a simulation protocol.

\section{A. Jamiołkowski fidelity}

The Jamiołkowski fidelity $F\left(U_{t}, \mathcal{E}_{t}\right)$ measures the closeness of one quantum operation to another. This fidelity is based on the Jamiołkowski isomorphism that was introduced in Section IVA that relates quantum operations to quantum states. The Jamiołkowski fidelity measures the overlap between two quantum operations by measuring the overlap between their corresponding states as defined by the Jamiołkowski isomorphism. This will be more rigorously defined below.

There are many ways that one might wish to go about defining fidelities for comparing quantum operations. The Jamiołkowski isomorphism is useful because it is easy to calculate, it satisfies some important mathematical properties, and it has several clear operational interpretations which are closely linked to the theory of quantum computation and simulation [58].

The first operational interpretation of the Jamiołkowski fidelity is in terms of a sampling quantum computation. Imagine that we were to implement a quantum operation, $U$, on some initial state $|x\rangle$ in order to attain measurement outcomes, $y$, on the output state. Say that we vary the input $x$ and sample in order to attain the joint probability distribution $p_{x}(y)$. Such a scenario is the way that one might perform any number of physically relevant simulation protocols. Say that the ideal distribution, that which occurs if $U$ is implemented perfectly, is $p_{x}(y)$ and the actual experimentally achieved distribution is $q_{x}(y)$, which occurs as a result of implementing and imperfect operation, $\mathcal{E}$. Then it can be shown [58] that the Jamiołkowski fidelity provides a lower bound on the overlap between the two probability distributions. That is,

$$
F(U, \mathcal{E}) \leq B\left(p_{x}(y), q_{x}(y)\right)
$$

where $B\left(p_{x}(y), q_{x}(y)\right)$ is the Bhattacharya overlap between the probability distributions and the problem in- stances are chosen uniformly at random. The Bhattacharya overlap is a measure for comparing probability distributions, while it doesn't have a succinct operational interpretation, it can be used to bound the Kolmogorov distance between two probability distributions.

Let us now consider a different simulation protocol, one that is based on a function computation. Imagine that we desire to use a unitary process, $U$, to simulate an evolution from the state $|x\rangle$ to the state $|f(x)\rangle$. We then perform measurements on the output state $|f(x)\rangle$ in order to determine the property of that state given by $f(x)$. If we instead performed the evolution $\mathcal{E}$ and output the state $\mathcal{E}(|x\rangle\langle x|)$, then the average probability, $p_{e}$, of getting an incorrect value of $f(x)$ given a uniform distribution over $x$ is bounded by,

$$
p_{e} \leq 1-F(U, \mathcal{E}) .
$$

More succinctly, if an experiment is designed to simulate some system in order to deterministically evaluate some property of a state, then the Jamiołkowski fidelity provides a bound on the average probability of an error.

We now move on to the more mathematically oriented properties of the Jamiołkowski fidelity. There are a number of ways of defining a fidelity for a quantum operation that are based on the Jamiolkowski isomorphism. In the case where we are always comparing an ideal unitary operation to a CP map it is convenient to use the following definition:

$$
F\left(\mathcal{E}_{t}, U_{t}\right)=F\left(E_{t},\left|\Psi_{t}\right\rangle\right)=\left\langle\Psi_{t}\left|E_{t}\right| \Psi_{t}\right\rangle,
$$

where

$$
\begin{aligned}
\left|\Psi_{t}\right\rangle & =\mathbb{1}^{(A)} \otimes U_{t}^{(B)}|\Phi\rangle, \\
E_{t} & =\mathcal{E}_{t}^{(B)}|\Phi\rangle_{A B}\langle\Phi|,
\end{aligned}
$$

and $|\Phi\rangle=1 / \sqrt{2^{m}} \sum_{k=0}^{2^{m}-1}|k\rangle_{A}|k\rangle_{B}$ is a maximally entangled state of $2 m$ qubits, where we labelled the basis of $m$ qubits by $\left\{|0\rangle,|1\rangle, \ldots\left|2^{m}-1\right\rangle\right\}$, i.e. identified the $m$ qubits with a $2^{m}$-dimensional system. The operation acts on one part of the maximally entangled state. This fidelity was called the Jamiołkowski process fidelity in [58], as it can be only requires elements of an operation's process matrix in order to be calculated. In this paper we simply refer to it as the Jamiołkowski fidelity.

It is worth noting that the Jamiołkowski fidelity is linearly related to the average fidelity, $\bar{F}(U, \mathcal{E})$, between a unitary operation $U$ and a $\mathrm{CP}$ map $\mathcal{E}$,

$$
\bar{F}(U, \mathcal{E})=\int d \psi\left\langle\psi\left|U^{\dagger} \mathcal{E}(|\psi\rangle\langle\psi|) U\right| \psi\right\rangle,
$$

where the integral is over the pure states $|\psi\rangle$. For a system of dimension $d$, the average fidelity can be calculated from the Jamiołkowski fidelity via,

$$
\bar{F}(U, \mathcal{E})=\frac{F(U, \mathcal{E}) d+1}{d+1} .
$$




\section{B. Simple bounds on the Jamiołkowski fidelity for simulation protocols}

The Jamiołkowski fidelity, as defined for this paper, can be used to generate several distance measures on the the set of all quantum operations. One such measure, $D\left(\mathcal{E}_{t}, U_{t}\right)$, will be used in this paper [58],

$$
D\left(\mathcal{E}_{t}, U_{t}\right)=\sqrt{1-F\left(\mathcal{E}_{t}, U_{t}\right)} .
$$

This distance measure is a metric on the space of quantum operations that is stable (that is remains unchanged) under the addition of ancillary quantum systems. Importantly, this measure also satisfies the following chaining inequality,

$$
D\left(U_{2} \circ U_{1}, \mathcal{E}_{2} \circ \mathcal{E}_{1}\right) \leq D\left(U_{1}, \mathcal{E}_{1}\right)+D\left(U_{2}, \mathcal{E}_{2}\right),
$$

where $U_{1,2}$ are unitary operations and $\mathcal{E}_{1,2}$ are tracepreserving quantum maps. This chaining inequality basically tells us that if we compare two sequences of quantum operations, then the distance between the two sequences will not diverge more rapidly than the distance between each individual step. This inequality turns out to be useful in our context, as quantum simulation protocols can often be cast in terms of sequences of unitary operations.

The chaining inequality allows us to obtain an upper bound on the distance (and correspondingly a lower bound on the fidelity) between two control sequences. Given an ideal unitary sequence $U=U_{m} \circ U_{m-1} \circ \cdots \circ U_{1}$ and the corresponding noisy sequence $\mathcal{E}=\mathcal{E}_{m} \circ \mathcal{E}_{m-1} \circ$ $\cdots \circ \mathcal{E}_{1}$ then the chaining inequality tells us,

$$
D(U, \mathcal{E}) \leq \sum_{j=1}^{m} D\left(U_{j}, \mathcal{E}_{j}\right) \leq m D_{\max }
$$

where $D_{\max }=\max _{j} D\left(U_{j}, \mathcal{E}_{j}\right)$.

Consider now the case where we wish to compare an ideal operation $U$ with a noisy operation of the form,

$$
\mathcal{E}=U \circ \mathcal{E}_{U}=\mathcal{E}_{U} \circ U .
$$

where $\mathcal{E}_{U}$ commutes with $U$. Using the definition of $F(U, \mathcal{E})$ it is easy to see that,

$$
F(U, \mathcal{E})=F\left(I, \mathcal{E}_{U}\right) .
$$

Correspondingly, we find

$$
D(U, \mathcal{E})=D\left(I, \mathcal{E}_{U}\right) .
$$

While this seems quite obvious, such circumstances occur readily in physical situations. For instance a system with Hamiltonian proportional to $\sigma_{z}^{\otimes m}$ undergoing dephasing, timing errors (as we will see later), or alternatively for $U$ and $\mathcal{E}$ that are both generated by fast control pulses (as is the case when using the Lie-Trotter summation formula).

Each of the simulation protocols discussed in this paper use sequences of unitary operations followed by measurements. The ideal unitary operations that appear in each protocol are either local unitary operations (often referred to as LU operations) or are applications of the entangling Hamiltonian $\sigma_{z} \otimes \sigma_{z}$ between pairs of the qubits in this system for some time $t$, that is the unitary $U_{t}$. In the case where we can consider all LU operations to be perfect it is very easy to establish upper (lower) bounds on the Jamiołkowski distance (fidelity) using the the chaining inequality.

A generic control sequence that appears in this paper looks something like,

$U=(L U)_{m} \circ U_{t} \circ(L U)_{m-1} \circ U_{t} \circ \cdots \circ(L U)_{2} \circ U_{t} \circ(L U)_{1}$,

where $(L U)_{j}$ denotes some local unitary operations and $U_{t}$ is an fixed entangling unitary that could in principle be applied between any set of qubits in the system. If we consider noise that only occurs as a result of the entangling operation $U_{t}$ then we can replace $U_{t}$ in the above sequence by some quantum map $\mathcal{E}_{t}$ that describes the noisy entangling operation and our noisy control sequence looks like,

$\mathcal{E}=(L \hat{U})_{m} \circ \mathcal{E}_{t} \circ\left(L U \hat{)}_{m-1} \circ \mathcal{E}_{t} \circ \cdots \circ(L \hat{U})_{2} \circ \mathcal{E}_{t} \circ(L \hat{U})_{1}\right.$.

Comparing these two sequences with the chaining inequality we find,

$$
\begin{aligned}
D(U, \mathcal{E}) \leq & D\left((L U)_{m},(L U)_{m}\right)+D\left(U_{t}, \mathcal{E}_{t}\right) \\
& +D\left((L U)_{m-1},(L U)_{m-1}\right)+D\left(U_{t}, \mathcal{E}_{t}\right)+\ldots
\end{aligned}
$$

If we note that for all $j D\left((L U)_{j},(L U)_{j}\right)=0$ then the above expression can be simplified to,

$$
D(U, \mathcal{E}) \leq k D\left(U_{t}, \mathcal{E}_{t}\right),
$$

for a sequence involving $k$ implementations of the map $\mathcal{E}_{t}$. Thus the problem of establishing an upper bound on the distance between $U$ and $\mathcal{E}$ is reduced to finding the distance between $U_{t}$ and $\mathcal{E}_{t}$. The corresponding fidelity bound is:

$$
F(U, \mathcal{E}) \geq 1-k^{2} D\left(U_{t}, \mathcal{E}_{t}\right)^{2}
$$

This bound is not the tightest bound that can always be established analytically. Later in this paper we shall see similar constructions that are optimized depending on on the simulation model that appears and the noise that is being modelled. Better bounds can be found by grouping the basic operations in each simulation control sequence that commute with each other before applying the chaining inequality. For instance, if all noise terms and unitary operations in a control sequence commute with one another the fidelity can be calculated precisely. Conversely, if each local unitary operation that appears does not commute with $U_{t}$ then it is not possible to find a better bound on the fidelity without resorting to further approximations.

While the Jamiołkowski fidelity is in general a suitable distance measure for noisy quantum operations, one has 
to be a bit careful about its interpretation. To be precise, when considering unitary operations $U_{t}$ with small $t$, i.e. time evolution with respect to the desired Hamiltonian for a small time $t$, the operation will be very close to the identity. The influence of noise, as resulting from a master equation description we consider here, also increases with time $t$. For small $t$, the influence of noise is hence very small, and also the noisy operation will be close to the identity. This will lead to a fidelity close to 1 , also in cases where the noise dominates over the Hamiltonian part of the evolution. In particular, it can happen that for small $t$ the fidelity is close to one, but the noisy operation is not capable of creating entanglement (i.e. the corresponding operator $E_{t}$ is separable [44]).

\section{Gate fidelity for long-time evolutions}

To allow for a better comparison, we thus also consider unitary gates generated by time evolutions for long times of $O(1)$. The ideal evolution is given by the unitary gate $U_{t}$, which is obtained by the time evolution with respect to the desired Hamiltonian $H$ for time $t$, where e.g. $t=\pi / 4$. The ideal evolution is compared with a sequence of basic noisy evolutions that approximate the desired gate. To be precise, the noisy evolution $\mathcal{E}_{\delta t}$ for (possibly small) times $\delta t$ is applied $n$ times, where $n=t / \delta t$, to approximate the desired unitary operation $U_{t}$. The Jamiołkowski fidelity

$$
F_{t}\left(\mathcal{E}_{\delta t}\right) \equiv F\left(\prod_{k=1}^{n} \mathcal{E}_{\delta t}, U_{t}\right)
$$

is a suitable quantity to measure the quality of $\mathcal{E}_{\delta t}$ and compare such noisy evolutions with different times $\delta t$. Also from a practical point of view, $F_{t}$ is a useful quantity, as in many cases the goal is to generate a certain unitary gate, and $F_{t}$ measures directly with which fidelity this is possible. It turns out that the optimal strategy is often not to generate very precise approximation of the desired evolutions for short times $\delta t$ and apply a sequence of many of such evolutions, but to chose some fixed intermediate time $\delta t^{\prime}$ which only require a moderate repetition of these basic evolutions.

\section{NOISE MODEL 1: MASTER EQUATION}

We will describe the influence of noise by a master equation of Lindblad form that is derived under certain assumptions, e.g. the Markov approximation. To be precise, we model the interaction between systems by a master equation of the form

$$
\frac{\partial}{\partial t} \rho=\mathcal{H} \rho+\mathcal{L} \rho
$$

where we have separated the evolution into two parts, a Hamiltonian part,

$$
\mathcal{H} \rho:=-i[H, \rho]
$$

corresponding to the ideal unitary process in question, and a noise part described by some Liouvillian superoperator $\mathcal{L}$. The formal solution of this master equation can be written as

$$
\rho(t)=e^{(\mathcal{H}+\mathcal{L}) t} \rho(0)
$$

which can, for small $t=\delta t$, be Taylor expanded

$$
\begin{aligned}
\rho(\delta t) \approx & \rho(0) \\
+ & \delta t(\mathcal{H}+\mathcal{L}) \rho(0) \\
+ & \frac{\delta t^{2}}{2}\left(\mathcal{H}^{2}+\mathcal{L}^{2}+\mathcal{L} \mathcal{H}+\mathcal{H} \mathcal{L}\right) \rho(0) \\
+ & O\left(\delta t^{3}\right) .
\end{aligned}
$$

In the following, we will often assume a coupling of the individual particles to independent thermal reservoirs, that is noise acts independently on particles. The Liouvillian $\mathcal{L}$ is in this case given by a sum of terms corresponding to different particles,

$$
\mathcal{L}=\sum_{\alpha} \mathcal{L}^{(\alpha)}
$$

For qubits, we describe the coupling of a single particle to a thermal reservoir by a quantum-optical noise term [57],

$$
\begin{aligned}
\mathcal{L}^{(\alpha)} \rho= & -\frac{B}{2}(1-s)\left[\sigma_{+}^{(\alpha)} \sigma_{-}^{(\alpha)} \rho+\rho \sigma_{+}^{(\alpha)} \sigma_{-}^{(\alpha)}-2 \sigma_{-}^{(\alpha)} \rho \sigma_{+}^{(\alpha)}\right] \\
& -\frac{B}{2} s\left[\sigma_{-}^{(\alpha)} \sigma_{+}^{(\alpha)} \rho+\rho \sigma_{-}^{(\alpha)} \sigma_{+}^{(\alpha)}-2 \sigma_{+}^{(\alpha)} \rho \sigma_{-}^{(\alpha)}\right] \\
& -\frac{2 C-B}{8}\left[2 \rho-2 \sigma_{z}^{(\alpha)} \rho \sigma_{z}^{(\alpha)}\right]
\end{aligned}
$$

with $\sigma_{ \pm}^{(\alpha)} \equiv 1 / 2\left(\sigma_{x}^{(\alpha)} \pm i \sigma_{y}^{(\alpha)}\right)$ act on particle $\alpha$ and $2 C \geq$ $B$. While parameters $B, C$ give the decay rate of inversion and polarization, $s \in[0,1]$ depends on the temperature $T$ of the bath. More precisely $s=\lim _{t \rightarrow \infty}\left\langle\frac{1+\sigma_{z}}{2}\right\rangle_{t}$, where $s=1 / 2$ corresponds to $T=\infty$. For $B=0$ and $C=\gamma$, this corresponds to dephasing noise, while for $s=1 / 2$ and $B=C \equiv \kappa$, we obtain white noise, corresponding to a depolarizing channel. Without interaction, i.e. for $H=0$, the corresponding time dependent completely positive maps obtained by integrating the master equation are given by a dephasing channel,

$$
\mathcal{D}^{(\alpha)} \rho=p(t) \rho+\frac{1-p(t)}{2}\left(\rho+\sigma_{3}^{(\alpha)} \rho \sigma_{3}^{(\alpha)}\right),
$$

with $p(t)=e^{-\gamma t}$ or a depolarizing channel,

$$
\mathcal{M}^{(\alpha)} \rho=p(t) \rho+\frac{1-p(t)}{4} \sum_{j=0}^{3} \sigma_{j}^{(\alpha)} \rho \sigma_{j}^{(\alpha)},
$$

with $p(t)=e^{-\kappa t}$ respectively. 


\section{A. Local noise equivalent}

We also use a third quantity to determine the quality of the simulation process, which is the local noise equivalent (LNE). Here, the noisy process described by $\mathcal{E}_{t}$ is compared with a (virtual) noisy process $\tilde{\mathcal{E}}_{t}$ that corresponds to an evolution with respect to a superoperator given by

$$
\mathcal{H}+\sum_{\alpha=1}^{m} \mathcal{L}^{(\alpha)}
$$

for time $t$, where the Hamiltonain part $\mathcal{H} \rho=-i[H, \rho]$ corresponds to the ideal evolution, while the noise part is described by individual (local) coupling of qubits to thermal baths (see Eq. 103). In particular, we consider the cases of dephasing noise $(B=0, C=\gamma)$ and white noise $(s=1 / 2, B=C \equiv \kappa)$. The parameter $\gamma[\kappa]$ determines the strength of coupling of each qubit to the reservoir, and is adjusted in such a way that the processes $\mathcal{E}_{t}$ and $\tilde{\mathcal{E}}_{t}=\exp \left[\left(\mathcal{H}+\sum_{\alpha=1}^{m} \mathcal{L}^{(\alpha)}\right) t\right]$ lead to the same Jamiołkowski fidelity with respect to the ideal unitary evolution $U_{t}$, i.e.

$$
F\left(\mathcal{E}_{t}, U_{t}\right)=F\left(\tilde{\mathcal{E}}_{t}, U_{t}\right)
$$

The coupling strength $\gamma[\kappa]$ for which Eq. 107 is fulfilled hence give the local noise equivalent, i.e. the amount of local noise that would lead to same fidelity after an evolution for time $t$. This provides a suitable measure for the quality of a noisy evolution. We remark that typically $\mathcal{E}_{t}$ and $\tilde{\mathcal{E}}_{t}$ will not be identical. Nevertheless, if the initial process containing two-body interactions is described by single qubit dephasing/depolarizing noise with some parameter $\gamma_{0}\left[\kappa_{0}\right]$, a LNE $\gamma>\gamma_{0}\left[\kappa>\kappa_{0}\right]$ can be considered as increase of noise level, where it is implicitly assumed by such a comparison that generation of a many-body interaction is as difficult as the generation of a two-body interaction (which might, however, not be the case). As we will see in the following, some methods typically lead to a LNE which is much larger than the initial $\gamma_{0}, \kappa_{0}$.

In certain cases, the LNE can be determined analytically from the Jamiołkowski fidelity. Consider e.g. the case where the ideal (desired) evolution is given by a Hamiltonian $H=\sigma_{z}^{\otimes m}$, and we consider dephasing noise on individual qubits described by a Liouvillian $\mathcal{L}^{(\alpha)} \rho=$ $-\gamma / 2\left(\rho-\sigma_{z}^{(\alpha)} \rho \sigma_{z}^{\alpha)}\right)$. In this case, the Jamiołkowski fidelity of the process $\tilde{\mathcal{E}}_{t}=\exp \left[\left(\mathcal{H}+\sum_{\alpha} \mathcal{L}^{(\alpha)}\right) t\right]=$ $\exp (\mathcal{H} t) \exp \left(\sum_{\alpha} \mathcal{L}^{(\alpha)} t\right)$ is given by

$$
F\left(\tilde{\mathcal{E}}_{t}, U_{t}\right)=F\left(\otimes_{\alpha} \mathcal{D}^{(\alpha)}, \mathbb{1}\right)=\left(\frac{1+p(t)}{2}\right)^{m},
$$

where $\mathcal{D}^{(\alpha)}$ is the dephasing channel defined in Eq. 104 with $p(t)=\exp (-\gamma t)$, and we used that Hamiltonian and noise parts commute. The relation of the LNE to the fidelity $F \equiv F\left(\mathcal{E}_{t}, U_{t}\right)$ of a process is in this case given by

$$
\gamma=-\frac{\ln \left(2 F^{1 / m}-1\right)}{t} .
$$

For other Hamiltonians, and depolarizing noise, where Hamiltonian and noise parts do not commute, it is often difficult to solve the corresponding master equation analytically. However, the LNE can be easily determined numerically also in these cases.

\section{NOISE MODEL 2: TIMING ERRORS}

As we have discussed, all of the Hamiltonian simulation methods in this article can be thought of as being implemented via control sequences of Hamiltonian evolutions. In these protocols we manipulate the amount of time that a Hamiltonian evolution is induced on a system. In this section we examine the effect of inaccuracies in this evolution time.

Say we wish to physically implement the unitary operation $U=e^{-i H t}$ and we give ourselves control over the amount of time that the evolution occurs. In reality, we can only control $t$ with finite precision. In a single instance of a controlled evolution by $H$ for some time $t$, we assume that there will be some Gaussian distributed random error $\delta$ with mean 0 . The corresponding completely positive map is given by,

$$
\mathcal{E}(\rho)=\int_{-\infty}^{\infty} d \delta p(\delta) e^{-i H(t+\delta)} \rho e^{i H(t+\delta)},
$$

where $p(\delta)$ is a Gaussian distribution.

Importantly, an error such as that described by Eq. 110 can be thought of as two distinct, commuting operations on the the state $\rho$. That is, we can define two super-operators $\mathcal{U}_{t}(\rho) \equiv e^{-i H t} \rho e^{i H t}$ and $\mathcal{T}(\rho) \equiv$ $\int_{-\infty}^{\infty} d \delta p(\delta) e^{-i H \delta} \rho e^{i H \delta}=\int_{-\infty}^{\infty} d \delta p(\delta) \mathcal{U}_{\delta}(\rho)$ such that,

$$
\mathcal{E}(\rho)=\mathcal{T} \circ \mathcal{H}(\rho)=\mathcal{H} \circ \mathcal{T}(\rho) .
$$

Noting this, we are free to think of the map given in Eq. 110 as being comprised of some "perfect" unitary operation, that is the operation $\mathcal{U}$, which is followed by a noisy operation, $\mathcal{T}$.

\section{Unitary Hamiltonians}

An evolution according to Eq. 110 has a particularly simple form in the special case where a Hamiltonian is of the form $H=\omega H_{\omega}$, where $H_{\omega}^{2}=\mathbb{1}$. This property allows us to express the unitary evolution generated by such Hamiltonians in the following simple form,

$$
e^{-i H t}=\cos (\omega t) \mathbb{1}-i \sin (\omega t) H_{\omega} .
$$


There are a number of interesting Hamiltonians in this class. For instance the two-qubit Ising interaction, as well as single-qubit Pauli rotations.

Utilizing the identity in Eq. 112, we can express the map $\mathcal{T}(\rho)$ as,

$$
\begin{aligned}
\mathcal{T}(\rho)= & \int_{-\infty}^{\infty} d \delta p(\delta)\left[\cos ^{2}(\omega \delta) \mathbb{1} \rho \mathbb{1}+\sin ^{2}(\omega \delta) H_{\omega} \rho H_{\omega}\right. \\
& \left.-i \sin (\omega \delta) \cos (\omega \delta)\left(H_{\omega} \rho \mathbb{1}-\mathbb{1} \rho H_{\omega}\right)\right] .
\end{aligned}
$$

As $p(\delta)$ is an even function of $\delta$ and the product $\sin (\omega \delta) \cos (\omega \delta)$ is an odd function, we know that,

$$
\int_{-\infty}^{\infty} p(\delta) \sin (\omega \delta) \cos (\omega \delta)=0 .
$$

Thus we find,

$$
\mathcal{T}(\rho)=\int_{-\infty}^{\infty} d \delta p(\delta) \cos ^{2}(\omega \delta) \mathbb{1} \rho \mathbb{1}+\sin ^{2}(\omega \delta) H_{\omega} \rho H_{\omega} .
$$

As we have assumed that the timing error is Gaussian distributed around zero we can write the probability distribution $p(\delta)$ in the following way,

$$
p(\delta)=\frac{1}{\sigma \sqrt{2 \pi}} e^{-\frac{\delta^{2}}{2 \sigma^{2}}},
$$

where $\sigma$ is the standard deviation of the probability distribution. We can find an analytic expression for $\mathcal{T}(\rho)$ by inserting Eq. 116] into Eq. 115, using some standard trigonometric identities, and noting the following integral identity,

$$
\int_{-\infty}^{\infty} d x e^{a x^{2}} \cos (b x)=\sqrt{\frac{\pi}{4 a}} e^{-\frac{b^{2}}{4 a}} .
$$

Doing this we find that the completely positive map associated with a random Gaussian error in the control time of a Hamiltonian is given by,

$$
\mathcal{T}(\rho)=\frac{1}{2}(q+1) \mathbb{1} \rho \mathbb{1}+\frac{1}{2}(1-q) H_{\omega} \rho H_{\omega}
$$

where $q(\sigma, \omega)=e^{-2 \sigma^{2} \omega^{2}}$. Equation 118 can be re-written as,

$$
\mathcal{T}(\rho)=q \rho+\frac{(1-q)}{2}\left(\rho+H_{\omega} \rho H_{\omega}\right) .
$$

which has a similar form to that previously seen for the de-phasing channel.

The corresponding Jamiołkowski fidelity a for random timing error on a unitary Hamiltonian would be,

$$
F(\mathcal{H}, \mathcal{E})=F(\mathbb{1}, \mathcal{T})=\frac{(1+q)}{2} .
$$

The corresponding Jamiołkowski distance is

$$
D(\mathcal{H}, \mathcal{E})=\sqrt{\frac{(1-q)}{2}} .
$$

Example: Consider the two-qubit Ising Hamiltonian, $H=\omega \sigma_{z} \otimes \sigma_{z}$. If we were to evolve a two-qubit system by $H$ to a system of two qubits for some time $t$, then the map associated with a random timing error on the evolution time would take the form:

$$
\mathcal{T}(\rho)=q \mathbb{1} \rho \mathbb{1}+\frac{(1-q)}{2}\left(\rho+\sigma_{z} \otimes \sigma_{z} \rho \sigma_{z} \otimes \sigma_{z}\right) .
$$

Such a map can be thought of as a collective dephasing channel. We shall use this example frequently in the remainder of this paper.

\section{Sums of commuting unitary Hamiltonians}

We can extend the results of the previous section to a wider variety of Hamiltonians. Consider the following Hamiltonian,

$$
H=\sum_{j=1}^{n} \omega_{j} H_{j},
$$

where each $H_{j}$ is a unitary Hamiltonian, $\omega_{j}$ is real, and any two $H_{j}$ commute. As each of the $H_{j}$ commute, an evolution according to such a Hamiltonian can be factorized into a sequence of $n$ unitary evolutions,

$$
e^{-i H t} \rho e^{i H t}=e^{-i \omega_{1} H_{1} t} \ldots e^{-i \omega_{n} H_{n} t} \rho e^{i \omega_{n} H_{n} t} \ldots e^{i \omega_{1} H_{1} t},
$$

or in terms of super-operators,

$$
\mathcal{U}_{t}(\rho)=\mathcal{U}_{t}^{1} \circ \ldots \circ \mathcal{U}_{t}^{n}(\rho) .
$$

The effect of a timing error on such an evolution can be analyzed in a fashion similar unitary Hamiltonians that we examined in the previous subsection. We can describe an evolution of $H$ with a timing error using the completely positive map $\mathcal{E}$ that was defined in Eq. 110 in the previous section. Like we saw in the previous section, we find that because all of the $H_{j}$ commute $\mathcal{E}=\mathcal{U}_{t} \circ \mathcal{T}_{H}=$ $\mathcal{T}_{H} \circ \mathcal{U}_{t}$. Hence in order to understand the effects of the timing error we only need to consider the nature of the operator $\mathcal{T}_{H}$.

In terms of superoperators, $\mathcal{T}_{H}$ can be written,

$$
\mathcal{T}_{H}(\rho)=\int_{-\infty}^{\infty} p(\delta) \mathcal{U}_{\delta}^{1} \circ \ldots \circ \mathcal{U}_{\delta}^{n}(\rho),
$$

where the order the sequence of $\mathcal{U}_{t}^{j}$ is irrelevant. Note that the integral in the expression for $\mathcal{T}_{H}$ is only over a single variable, $\delta$. As we did in the previous section, we can expand each of the $\mathcal{U}_{\delta}^{j}$ terms in $\mathcal{T}_{H}$ using Eq. 112 and integrating out odd terms in the expansion. That is,

$$
\begin{aligned}
\mathcal{T}_{H}(\rho)= & \int_{-\infty}^{\infty} p(\delta) \mathcal{U}_{\delta}^{1} \circ \ldots \circ \mathcal{U}_{\delta}^{n-1} \\
& \left(\cos ^{2}\left(\omega_{n} \delta\right) \mathbb{1} \rho \mathbb{1}+\sin ^{2}\left(\omega_{n} \delta\right) H_{n} \rho H_{n}\right) .
\end{aligned}
$$


Some algebra demonstrates that if each $\mathcal{U}_{\delta}^{j}$ is expanded in this way, then the resulting expression becomes quite complicated and writing it here would be unhelpful. Generally, the expression for $\mathcal{T}_{H}$ involves a sum of all products of the unitary Hamiltonians $H_{j}$. The nature of such an expression depends greatly on what each of the Hamiltonians $H_{j}$ actually are. This is best illustrated by an example.

Example: Imagine we wish to apply an Ising Hamiltonian to 3 qubits of the form:

$$
H=\omega \sigma_{z}^{1} \sigma_{z}^{2}+\omega \sigma_{z}^{1} \sigma_{z}^{3},
$$

for a time $t$. The resulting timing error operation can be written,

$\mathcal{T}_{H}(\rho)=\int_{-\infty}^{\infty} d \delta p(\delta)\left(e^{-i \omega \delta \sigma_{z}^{1} \sigma_{z}^{2}} e^{-i \omega \delta \sigma_{z}^{1} \sigma_{z}^{3}} \rho e^{i \omega \delta \sigma_{z}^{1} \sigma_{z}^{2}} e^{i \omega \delta \sigma_{z}^{1} \sigma_{z}^{3}}\right)$

This can be expanded in the fashion described above,

$$
\begin{aligned}
\mathcal{T}_{H}(\rho)= & \int_{-\infty}^{\infty} d \delta p(\delta)\left[\cos ^{4}(\omega \delta) \mathbb{1} \rho \mathbb{1}\right. \\
& +\cos ^{2}(\omega \delta) \sin ^{2}(\omega \delta)\left(\sigma_{z}^{1} \sigma_{z}^{2} \rho \sigma_{z}^{1} \sigma_{z}^{2}+\sigma_{z}^{1} \sigma_{z}^{3} \rho \sigma_{z}^{1} \sigma_{z}^{3}\right) \\
& \left.+\sin ^{4}(\omega \delta) \sigma_{z}^{2} \sigma_{z}^{3} \rho \sigma_{z}^{2} \sigma_{z}^{3}\right] .
\end{aligned}
$$

This expression can be simplified to,

$$
\begin{aligned}
\mathcal{T}_{H}(\rho)= & g \mathbb{1} \rho \mathbb{1}+(1-g)\left(\sigma_{z}^{1} \sigma_{z}^{2} \rho \sigma_{z}^{1} \sigma_{z}^{2}+\sigma_{z}^{2} \sigma_{z}^{3} \rho \sigma_{z}^{2} \sigma_{z}^{3}\right. \\
& +(1-g)^{2} \sigma_{z}^{1} \sigma_{z}^{3} \rho \sigma_{z}^{1} \sigma_{z}^{3}
\end{aligned}
$$

where

$$
g=\int_{-\infty}^{\infty} d \delta p(\delta) \cos ^{4}(\omega \delta)
$$

Assuming that $p(\delta)$ is a Gaussian distribution with mean 0 it can be written as it is in Equation [116 and we find an analytic expression for $g$,

$$
g=\frac{3}{2^{3}}+\frac{1}{2} e^{-2 \sigma^{2} \omega^{2}}+\frac{1}{2^{3}}\left(e^{-2 \sigma^{2} \omega^{2}}\right)^{4} .
$$

In this example, the Jamiołkowski fidelity is simply, $F(\mathcal{H}, \mathcal{E})=g$.

From this example we see for any $H$ that is a sum of commuting unitary Hamiltonians that it is possible to derive a lower bound on the Jamiołkowski fidelity given, a random timing error. Expanding $\mathcal{T}_{H}$ we find that there is always a term with the following form:

$$
I_{H}=\int_{-\infty}^{\infty} d \delta p(\delta) \prod_{j=1}^{n} \cos ^{2}\left(\omega_{j} \delta\right) \mathbb{1}^{\otimes n} \rho \mathbb{1}^{\otimes n}
$$

This means that the Jamiołkowski fidelty must be at least,

$$
F(\mathcal{H}, \mathcal{E}) \geq \int_{-\infty}^{\infty} d \delta p(\delta) \prod_{j=1}^{n} \cos ^{2 n}\left(\omega_{j} \delta\right)
$$

If each $\omega_{j}=\omega$ and $p(\delta)$ is a Gaussian, then it is possible to find an analytic form for this lower bound by using basic trigonometric identities,

$$
F(\mathcal{H}, \mathcal{E}) \geq \frac{1}{2^{2 n}}\left(\begin{array}{c}
2 n \\
n
\end{array}\right)+\frac{1}{2^{2 n-1}} \sum_{k=0}^{n-1}\left(\begin{array}{c}
2 n \\
k
\end{array}\right) e^{-2(n-k)^{2} \sigma^{2} \omega^{2}}
$$

As our three-qubit Ising model example demonstrates, this lower bound on the fidelity can be saturated. Saturation of this inequality occurs when no product of the Hamiltonians $H_{j}$ are equal to the identity. That is, the only term in $\mathcal{T}_{H}$ which gives a superoperator proportional to $\mathbb{1}$ is $I_{H}$. There are a many Hamiltonians that have this property. Two examples include 1-D Ising chains with open boundary conditions and Ising interaction Hamiltonians whose interaction pattern forms a star graph.

The Hamiltonian $H=\sum_{j=1}^{n} \omega_{j} H_{j}$ can be simulated by a sequence of $n$ independent evolutions of the Hamiltonians $H_{j}$. If each of these evolutions had a timing error then Eq. 126 would have $n$ independent $\delta_{j}$ terms over which one would integrate. This is equivalent to applying $n$ different error maps like that given in Eq. 119 . That is the timing error map would be given by a product of maps like $\mathcal{T}_{H_{1}} \ldots \mathcal{T}_{H_{n}}$. The fidelity of a timing error in such a case is then lower bounded by

$$
F(\mathcal{H}, \mathcal{E})=F\left(\mathbb{1}, \mathcal{T}_{H_{1}} \ldots \mathcal{T}_{H_{n}}\right) \geq \prod_{j=1}^{n}\left(\frac{1+q_{j}}{2}\right)
$$

This bound on the fidelity is saturated for the same $H$ as the bound derived for $\mathcal{T}_{H}$ above.

If we consider the situation where the above fidelity bounds are saturated, we can compare the effect of timing errors on an evolution according $H$ alone versus the effect of simulating $H=\sum_{j=1}^{n} \omega H_{j}$ by $n$ evolutions according to its constituent Hamiltonians $H_{j}$. For simplicity, we consider the case where $\omega_{j}=\omega$ and the standard deviation in the timing error for implementing $H$ is the same as for implementing each of it's constituent terms $H_{j}$. The resulting fidelities are given by the following integrals;

$$
F\left(\mathbb{1}, \mathcal{T}_{H}\right)=\int_{-\infty}^{\infty} d \delta p(\delta) \cos ^{2 n}(\omega \delta)
$$

for the case where we implement $H$ directly; and

$$
F\left(\mathbb{1}, \mathcal{T}_{H_{1}} \ldots \mathcal{T}_{H_{n}}\right)=\left(\int_{-\infty}^{\infty} d \delta p(\delta) \cos ^{2}(\omega \delta)\right)^{n}
$$

for the case where $H$ is implemented by evolutions according to the Hamiltonians $H_{j}$. We have reverted to writing these expressions in their integral form because in this form it is simpler to see how to apply Hölder's inequality to them. Recall Hölder's inequality,

$$
\int_{a}^{b}|\zeta(x) \eta(x)| d x \leq\left(\int_{a}^{b}|\zeta(x)|^{r} d x\right)^{\frac{1}{r}}\left(\int_{a}^{b}|\eta(x)|^{w} d x\right)^{\frac{1}{w}}
$$


where $\zeta(x), \eta(x)$ are integrable complex functions and $r, w>1$ satisfying

$$
\frac{1}{r}+\frac{1}{w}=1
$$

We set $\zeta(\delta)=p(\delta)^{\frac{n-1}{n}}, \eta(\delta)=p(\delta)^{\frac{1}{n}} \cos ^{2}(\omega \delta), r=\frac{n}{n-1}$ and $w=n$ and substitute these values into Hölder's inequality ${ }^{2}$. Then, noting that $p(\delta)$ and $\cos ^{2}(\omega \delta)$ are both always positive we find that

$$
\left(\int_{-\infty}^{\infty} d \delta p(\delta) \cos ^{2}(\omega \delta)\right)^{n} \leq \int_{-\infty}^{\infty} d \delta p(\delta) \cos ^{2 n}(\omega \delta)
$$

Thus we find that for $\delta=\delta_{j}$ and $\omega_{j}=\omega$ for all $j$

$$
F\left(\mathbb{1}, \mathcal{T}_{H}\right) \geq F\left(\mathbb{1}, \mathcal{T}_{H_{1}} \ldots \mathcal{T}_{H_{n}}\right)
$$

\section{COMMUTATOR METHOD}

We will now analyze the influence of noise, described by some master equation of Lindblad form, on the simulation of many-body interaction Hamiltonians generated by using the commutator method. The commutator method is based on a sequential application of evolutions for times $\delta t$ with respect to different Hamiltonians in such a way that, when performing a Taylor expansion in $\delta t$, all first order terms vanish and the second order terms that include the commutator of the initial Hamiltonians and hence many-body interaction terms- remain. The analysis is usually done at the level of operators (see Sec. II), where one obtains (up to higher order corrections) an effective Hamiltonian given by $H_{\mathrm{eff}}=-i / 2\left[H_{1}, H_{2}\right]$ which appears in second order $\delta t$. One can formally perform the analysis also at the level of superoperators, as is required when considering noisy interactions described by master equations.

\section{A. Three-body interactions}

We consider a sequence of four operations, each applied for time $\delta t$, where we denote the Hamiltonian part of the $j^{\text {th }}$ operation by $\mathcal{H}_{j}$, and the Liouvillian part by $\mathcal{L}_{j}$. An initial state $\rho(0)$ has evolved under this sequence of operations after a total time $t=4 \delta t$ as follows

$$
\rho(t)=e^{\left(\mathcal{H}_{4}+\mathcal{L}_{4}\right) \delta t} e^{\left(\mathcal{H}_{3}+\mathcal{L}_{3}\right) \delta t} e^{\left(\mathcal{H}_{2}+\mathcal{L}_{2}\right) \delta t} e^{\left(\mathcal{H}_{1}+\mathcal{L}_{1}\right) \delta t} \rho(0) .
$$

\footnotetext{
${ }^{2}$ M.B. would like to thank Otfried Gühne for pointing out this choice of variables.
}

For small $\delta t$, Eq. 144 can be Taylor expanded using Eq. 101, and one obtains

$$
\begin{aligned}
\rho(t)= & \rho(0)+\delta t\left(\sum_{j=1}^{4} \mathcal{H}_{j}+\sum_{j=1}^{4} \mathcal{L}_{j}\right) \rho(0) \\
& +\frac{\delta t^{2}}{2}\left(\sum_{j=1}^{4} \mathcal{H}_{j}^{2}+2 \sum_{l=2}^{4} \sum_{j=1}^{l-1} \mathcal{H}_{l} \mathcal{H}_{j}\right) \rho(0) \\
& +\frac{\delta t^{2}}{2} \sum_{l=2}^{4} \sum_{j=1}^{l-1} 2\left(\mathcal{H}_{l} \mathcal{L}_{j}+\mathcal{L}_{l} \mathcal{H}_{j}+\mathcal{L}_{l} \mathcal{L}_{j}\right) \rho(0) \\
& +\frac{\delta t^{2}}{2} \sum_{j=1}^{4}\left(\mathcal{L}_{j}^{2}+\mathcal{H}_{j} \mathcal{L}_{j}+\mathcal{L}_{j} \mathcal{H}_{j}\right) \rho(0) \\
& +O\left(\delta t^{3}\right)
\end{aligned}
$$

As for ideal evolutions, we consider the case where $\mathcal{H}_{4}=-\mathcal{H}_{2}, \mathcal{H}_{3}=-\mathcal{H}_{1}$, i.e. $H_{4}=-H_{2}, H_{3}=-H_{1}$. In this case, $\sum_{j=1}^{4} \mathcal{H}_{j} \rho(0)=0$, and the second line of Eq. 145 simplifies to $2 \delta t^{2} \tilde{\mathcal{H}} \rho(0)$ with $\tilde{\mathcal{H}} \rho=-i[\tilde{H}, \rho]$ and $\tilde{H}=\frac{-i}{2}\left[H_{1}, H_{2}\right]$. Also some of the terms in lines 3 and 4 of Eq. 145 are simplified. Note, however, that the term $\delta t \sum_{j=1}^{4} \mathcal{L}_{j} \rho(0)$-which is first order in $\delta t$ - remains. While $\mathcal{H}_{j}$ correspond to coherent processes that can annihilate each other, $\mathcal{L}_{j}$ correspond to incoherent noise processes, where no such interference is possible. This implies that noise appears in first order $\delta t$, while the (desired) Hamiltonian part only appears in second order, $\delta t^{2}$. We denote additional noise terms of lines 3 and 4 that appear in second order, $\delta t^{2}$, in Eq. 145 by $\frac{\delta t^{2}}{2} \mathcal{L}^{\prime}$, and use the short-hand notation $\overline{\mathcal{L}}=\sum_{j=1}^{4} \mathcal{L}_{j}$. The total time evolution can hence be written as

$$
\begin{aligned}
\rho(t) & =\rho(0)+2 \delta t^{2} \tilde{\mathcal{H}}+2 \delta t^{2}\left(\frac{\overline{\mathcal{L}}}{2 \delta t}+\frac{\mathcal{L}^{\prime}}{4}\right)+O\left(\delta t^{3}\right) \\
& =\exp \left[2 \delta t^{2}\left(\tilde{\mathcal{H}}+\frac{\overline{\mathcal{L}}}{2 \delta t}+\frac{\mathcal{L}^{\prime}}{4}\right)\right] \rho(0)+O\left(\delta t^{3}\right) \\
& =\exp \left[\delta t^{\prime}\left(\tilde{\mathcal{H}}+\frac{\overline{\mathcal{L}}}{\sqrt{2 \delta t^{\prime}}}+\frac{\mathcal{L}^{\prime}}{4}\right)\right] \rho(0)+O\left(\delta t^{\prime 3 / 2}\right) .
\end{aligned}
$$

where we have introduced, as in the noiseless case, a dilated time $\delta t^{\prime}=2 \delta t^{2}$. That is, when applying the sequence of evolutions specified in Eq. 144 for a total time of $4 \delta t$, this is equivalent (up to higher order corrections), to an evolution with respect to a Liouvillian superoperator

$$
\tilde{\mathcal{H}}+\frac{\overline{\mathcal{L}}}{\sqrt{2 \delta t^{\prime}}}+\frac{\mathcal{L}^{\prime}}{4}
$$

for a time $\delta t^{\prime}=2 \delta t^{2}$. Note that the Hamiltonian part of the evolution is given by $\tilde{H}=\frac{1}{2}\left[H_{1}, H_{2}\right]$ which provides the desired many-body interaction term, but the noise part of the evolution, dominated by $\overline{\mathcal{L}} / \sqrt{2 \delta t^{\prime}}$ is enhance 
by a factor of $\left(2 \delta t^{\prime}\right)^{-1 / 2}$, or equivalently $(2 \delta t)^{-1}$, as compared to the two-body evolutions. This is a significant increase of noise level, which clearly limits the applicability of the commutator method. In particular, the increase of noise level becomes larger for smaller times $\delta t$, while on the other hand smaller $\delta t$ increase the precision with which the desired many-body interaction terms are generated. Recall that the commutator method is based on validity of Taylor expansion, which requires small $\delta t$ and precision increases with decreasing $\delta t$. In other words, the dilation factor in simulation time given by the time cost, translates in the case of noisy interactions directly into an increase of noise level.

Example: We consider in the following a simple example that illustrates the increase of noise level. We consider the case where, independent of the interaction Hamiltonian, the Liouvillian describing the noise part of the evolution corresponds to single-qubit dephasing noise, i.e. is given by

$$
\sum_{\alpha} \mathcal{L}^{(\alpha)} \rho=\sum_{\alpha} \gamma_{0} / 2\left(\rho-\sigma_{z}^{(\alpha)} \rho \sigma_{z}^{(\alpha)}\right),
$$

for all particles $\alpha$ that are involved in an interaction. For Hamiltonians $H_{1}, H_{2}$ given in Eq. 10 and $H_{3}=$ $-H_{1}, H_{4}=-H_{2}$, the effective evolution resulting from the sequence of evolutions specified in Eq. 144 corresponds -in the ideal case- to an evolution with respect to the effective three-body Hamiltonian

$$
H_{\mathrm{eff}}=\sigma_{z}^{\otimes 3}
$$

for time $\delta t^{\prime}=2 \delta t^{2}$. When $H_{1}$ is applied the corresponding Liouvillian is given by $\mathcal{L}_{1}=\mathcal{L}^{(A)}+\mathcal{L}^{(B)}$. If $\delta t \ll 1$ and $\gamma_{0}<<1$ then the evolution $\mathcal{H}_{1}+\mathcal{L}_{1}$ corresponds to an evolution according to the Hamiltonian $H_{1}$ and a single-qubit dephasing channels acting on qubits $A$ and $B$. The resulting fidelity is given by

$$
F_{1}=\left(\frac{1+p}{2}\right)^{2}
$$

where $p=e^{-\gamma_{0} \delta t}$. Assuming the same fidelities for each of the two-qubit operations used to simulate $H_{\text {eff }}$ we can use the simple fidelity bound derived in the previous section, Eq. 96. to give a lower bound on the fidelity of the simulation

$$
F \geq 1-16 \gamma_{0} \delta t
$$

Note that this bound is only valid for small $\delta t$ and $\gamma_{0}$. In terms of the effective simulation time $\delta t^{\prime}$ it reads,

$$
F \geq 1-\frac{16}{\sqrt{2}} \gamma_{0} \sqrt{\delta t^{\prime}}
$$

We improve on this bound below by directly calculating the fidelity under similar approximations.

The effective Liouvillian $\mathcal{L}_{\text {eff }}$ describing the noise part of this simulation is dominated by the term $\frac{\overline{\mathcal{L}}}{\sqrt{2 \delta t^{\prime}}}=$ $\frac{\sum_{k} \mathcal{L}_{k}}{\sqrt{2 \delta t^{\prime}}}$, where $\mathcal{L}_{1}=\mathcal{L}_{3}=\mathcal{L}^{(A)}+\mathcal{L}^{(B)}, \mathcal{L}_{2}=\mathcal{L}_{4}=$ $\mathcal{L}^{(B)}+\mathcal{L}^{(C)}$ as only qubits $A B$ or $B C$ are involved in a particular operation. We thus obtain

$$
\mathcal{L}_{\mathrm{eff}} \approx \frac{2 \mathcal{L}^{(A)}+2 \mathcal{L}^{(C)}+4 \mathcal{L}^{(B)}}{\sqrt{2 \delta t^{\prime}}} .
$$

In principle, the sequence of operations leading to the effective Hamiltonian $H_{\text {eff }}$ can be symmetrized by permuting the roles of qubits $A, B, C$, either probabilistically or sequentially, which leads to symmetrized noise in the effective Liouvillian

$$
\mathcal{L}_{\mathrm{eff}, \mathrm{sym}} \approx \frac{8 / 3\left(\mathcal{L}^{(A)}+\mathcal{L}^{(B)}+\mathcal{L}^{(C)}\right)}{\sqrt{2 \delta t^{\prime}}},
$$

where the factor $8 / 3$ results from $(2 \times 2+1 \times 4) / 3$, i.e. in two out of three cases we obtain a factor 2 in front of the Liouvillian term (role of particles $A, C$ ), while in 1 out of 3 cases there is a factor 4 (role of particle $B$ ) - see Eq. 153. The LNE with respect to dephasing noise can be directly read off from above equation and is (approximately) given by

$$
\gamma \approx \frac{8}{3 \sqrt{2 \delta t^{\prime}}} \gamma_{0}
$$

where the expressions are only valid for $\delta t^{\prime} \ll 1$. The Jamiołkowski fidelity can be estimated via Eq. 108, and one obtains

$$
F \approx\left(\frac{1+e^{-\sqrt{\delta t^{\prime}} 8 \gamma_{0} /(3 \sqrt{2})}}{2}\right)^{3} \approx 1-2 \sqrt{2 \delta t^{\prime}} \gamma_{0}
$$

We remark that dephasing noise is in many cases not an appropriate model, and also the assumption that dephasing noise occurs independent of the Hamiltonian might often not be fulfilled. Consider e.g. the case where a basic two-body Hamiltonian $H=\sigma_{z} \sigma_{z}$ is manipulated by means of local unitary operations to simulate a new effective two-body Hamiltonian, e.g. $H^{\prime}=\sigma_{x} \sigma_{x}$. In this case, the dephasing noise is also transformed, and one obtains bit-flip noise for the new effective Hamiltonian instead.

A similar calculation can be performed for depolarizing (white) noise, i.e. the Liouvillian describing the noise part is a sum of local terms, $\sum_{\alpha} \mathcal{L}^{(\alpha)}$, with $\mathcal{L}^{(\alpha)}$ given by Eq. 103 with $s=1 / 2$ and $B=C \equiv \kappa$. Again, one finds

$$
\kappa \approx \frac{8}{3 \sqrt{2 \delta t^{\prime}}} \kappa_{0}
$$

for the LNE, while for the fidelity we can simply bound it in the limit of small $\kappa$ and $\delta t$ using Eq. 96 by

$$
F \geq 1-\frac{24}{\sqrt{2}} \kappa_{0} \sqrt{\delta t^{\prime}}
$$

and we can explicitly estimate the fidelity in the same limits to be

$$
F \approx\left(\frac{1+3 e^{-\sqrt{\delta t^{\prime}} \kappa_{0} /(8 \sqrt{2})}}{4}\right)^{3} \approx 1-3 \sqrt{2 \delta t^{\prime}} \kappa_{0} .
$$


Above calculations should be considered as estimates of LNE and fidelity. A complete calculation involves also additional errors due to Taylor expansion and noise terms occurring in order $\delta t^{\prime}$. We have thus performed numerical simulations taking all errors into account, and considered the (often) more realistic model of white noise (depolarizing channels). The results of these simulations for the generation of an effective three-body interaction are shown in Figs. 556. Plots correspond to coupling strength $\kappa$ that would lead to single qubit white noise channels with parameter $p=0.9 ; 0.99 ; 0.999$ respectively when applied for time $\delta t=\pi$ (see Eq. 105). The results fully agree with the analytic estimates (see Eqs. 157]159) for small $\delta t^{\prime}$. For larger $\delta t^{\prime}$, higher order corrections due to Taylor expansion also play a role and further increase the noise level (and hence increase the LNE and reduce the fidelity). We remark that although for small $\delta t^{\prime}$ the fidelity is close to unity, the quality of the simulation is nevertheless very low as the local noise equivalent is enhanced significantly.

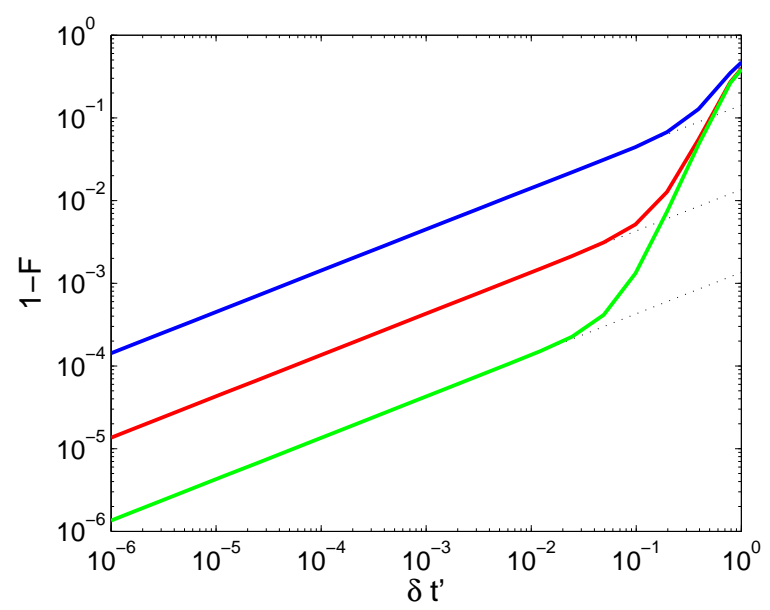

FIG. 5: (color online) Error of three-qubit interaction $\exp \left(-i \delta t^{\prime} \sigma_{z}^{\otimes 3}\right)$ applied for time $\delta t^{\prime}$ generated by commutator method. Plot shows deviation from ideal evolution measured by the fidelity, $1-F$, as a function of $\delta t^{\prime}$. Curves from top to bottom correspond to $p=0.9$ (blue), $p=0.99$ (red), $p=0.999$ (green), where $p=\exp (-\pi \kappa)$. Dotted lines are analytic estimates of Eq. 159, $1-F=3 \sqrt{2 \delta t^{\prime}} \kappa_{0}$.

\section{B. Timing errors}

In Section VII we derived CP maps that describe the effect of random timing errors in the implementation of a Hamiltonian evolution. As we saw in Section II we can simulate $H_{\mathrm{eff}}=\sigma_{z} \otimes \sigma_{z} \otimes \sigma_{z}$ by a control sequence involving the Hamiltonians $\pm H_{1}= \pm \sigma_{z}^{(A)} \sigma_{z}^{(B)}$ and $\pm H_{2}= \pm \sigma_{y}^{(B)} \sigma_{z}^{(C)}$. Both $H_{1}$ and $H_{2}$ are unitary and Hermitian, thus they are their own inverses. In Sec-

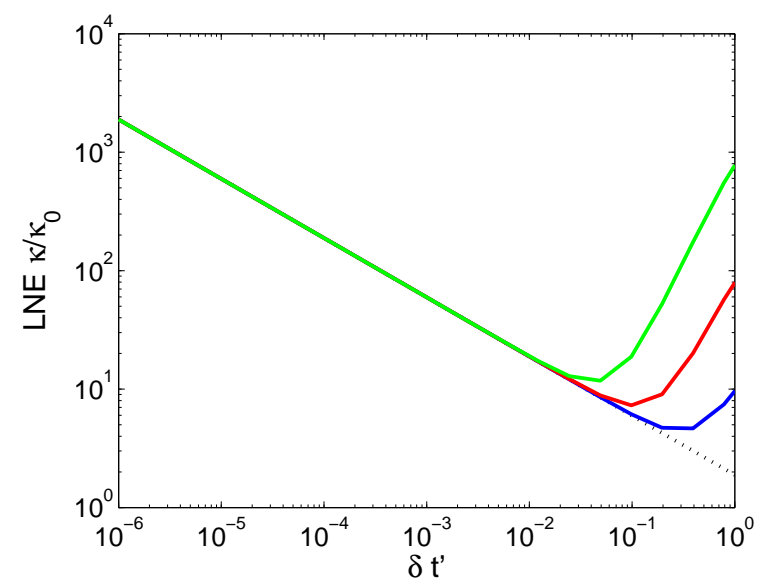

FIG. 6: (color online) Increase of Local noise equivalent of three-qubit interaction $\exp \left(-i \delta t^{\prime} \sigma_{z}^{\otimes 3}\right)$ applied for time $\delta t^{\prime}$ generated by commutator method. Plot shows $\kappa / \kappa_{0}$, as a function of $\delta t^{\prime}$. Curves from bottom to top correspond to $p=0.9$ (blue), $p=0.99$ (red), $p=0.999$ (green), where $p=\exp (-\pi \kappa)$. Dotted line is the analytic estimate given in Eq. 157] $\kappa / \kappa_{0}=\frac{8}{3 \sqrt{2 \delta t^{\prime}}}$.

tion VII (Eq. 120) we explicitly calculated the resulting fidelity of a timing error for such Hamiltonians. Considering such a noise model and given that $\delta t<<1$ then we can use Eq. 96 to estimate a lower bound on the resulting fidelity. Assuming additionally that the standard deviation, $\sigma$, of each timing error is the same we find the following lower bound on the fidelity of the simulation of $H_{\text {eff }}$ to be

$$
F \geq 1-8(1-q)
$$

where $q=e^{-2 \sigma^{2}}$.

\section{Many-body interactions}

It is straightforward to perform a similar analysis for many-body interactions involving more than three qubits. Essentially, one finds that the time cost of simulating many-body Hamiltonians using basic two-body interactions translates to an increase in noise level. As shown in Sec. II the desired $m$-body Hamiltonian appears in $\delta t_{m}=O\left(\delta t^{(m-1)}\right)$, where we have denoted by $\delta t_{m}$ the time for which the effective $m$-body Hamiltonian is applied. The noise, however, still appears in first order $\delta t$, or equivalently $O\left(\delta t_{m}^{1 /(m-1)}\right)$. This corresponds to an increase of noise level by a factor of order $O\left(\delta t^{-(m-2)}\right)$, or equivalently $O\left(\delta t_{m}^{-(m-2) /(m-1)}\right)$. The total evolution for time $\delta t_{m}$ is thus essentially governed by a Liouvillian superoperator of the form

$$
\tilde{\mathcal{H}}+O\left(\delta t_{m}^{-(m-2) /(m-1)}\right) \overline{\mathcal{L}}
$$


where $\tilde{\mathcal{H}}=-i\left[H_{\text {eff }}, \rho\right]$ and $H_{\text {eff }}$ is the desired $m$-body Hamiltonian, while $\overline{\mathcal{L}}$ is given by an appropriate sum of Liouvillians describing noise in two-body interactions involved in the process. This noise is enhanced by a factor of $O\left(\delta t_{m}^{-(m-2) /(m-1)}\right)$.

The LNE also increases accordingly, i.e.

$$
\gamma \approx O\left(\delta t_{m}^{-(m-2) /(m-1)}\right) \gamma_{0}
$$

while the fidelity is approximately given by

$$
F \approx 1-m \gamma_{0} \times O\left(\delta t_{m}^{-(m-2) /(m-1)}\right) .
$$

In above expressions, we have again assumed a simple model with only dephasing noise specified by a coupling constant $\gamma_{0}[\gamma]$ respectively. For large $m$, the dilation factor approaches $\delta t_{m}^{-1}$ and can be significant. In particular, the resulting CPM may no longer be capable of creating entanglement for all times.

We have also performed a full numerical analysis taking all errors and imperfections into account. We find that qualitative scaling for small simulation time $\delta t_{m}$ is essentially as described above. For larger $\delta t_{m}$ additional errors due to Taylor expansion and/or noise terms in higher order $\delta t$ further increase the noise level.

\section{Simulation of strongly entangling multi-qubit gate}

Above considerations are concerned with the generation of a basic $m$-body Hamiltonian of the form $H_{\text {eff }}=$ $\sigma_{z}^{\otimes m}$ for time $\delta t_{m}$. However, such a basic $m$-body Hamiltonian only serves as a building block to generate via standard Hamiltonian simulation techniques- other many-body Hamiltonians, or multi-qubit gates. To illustrate the accuracy of such simulation in the presence of noise, we consider two examples:

(i) The simulation of the three-body Hamiltonian $H_{1}=\sigma_{z}^{(1)} \sigma_{z}^{(2)} \sigma_{z}^{(3)}$ for time $t=\pi / 4$;

(ii) The simulation of the three-body Hamiltonian $H_{2}=\sigma_{z}^{(1)} \sigma_{z}^{(2)} \sigma_{z}^{(3)}+\sum_{k=1}^{3} \sigma_{x}^{(k)}$ for time $t=\pi / 4$

Example (i) only contains the basic Hamiltonian, while example (ii) also includes single-qubit terms and the application of Hamiltonian simulation techniques to generate sequentially the two non-commuting terms $\sigma_{z}^{\otimes 3}$ and $\sum_{k=1}^{3} \sigma_{x}^{(k)}$. Again, we determine fidelity and LNE as a function of the time $\delta t_{m}$ for which the basic two-body interaction Hamiltonian is generated. Small times $\delta t_{m}$ require several application (and additional intermediate local unitary operations) of the basic 3-body Hamiltonian to achieve an evolution for time $t=\pi / 4$, while larger times $\delta t_{m}$ require fewer applications but involve larger Taylor expansion errors in both the generation of the basic 3-body Hamiltonian and the simulation of the desired Hamiltonian $H$ from $H_{\text {eff }}$. The results of the numerical simulation for the Hamiltonian (ii) are shown in
Fig. 7 for different values of the white noise parameter $p$, where $p=\exp (-\pi \kappa)$. That is, the corresponding coupling strength to the thermal bath $\kappa$ is such that the evolution with respect to the noise part of the Liouvillian for time $t=\pi$ leads to a depolarizing map (see Eq. 105) with parameter $p$. One observes that there exists an optimal time $\delta t_{m}$ that leads to a maximal gate fidelity, and the optimal value of $\delta t_{m}$ depends on $p$. Recall that the LNE to generate the basic 3-body hamiltonian increases with decreasing $\delta t_{m}$ (see Fig. 6), while $\delta t_{m}$ cannot be chosen too big to limit Taylor expansion errors. The optimal value of $\delta t_{m}$ is hence a compromise between the two competing requirements. The results for simulation of the Hamiltonian (i) are very similar and are not shown here.

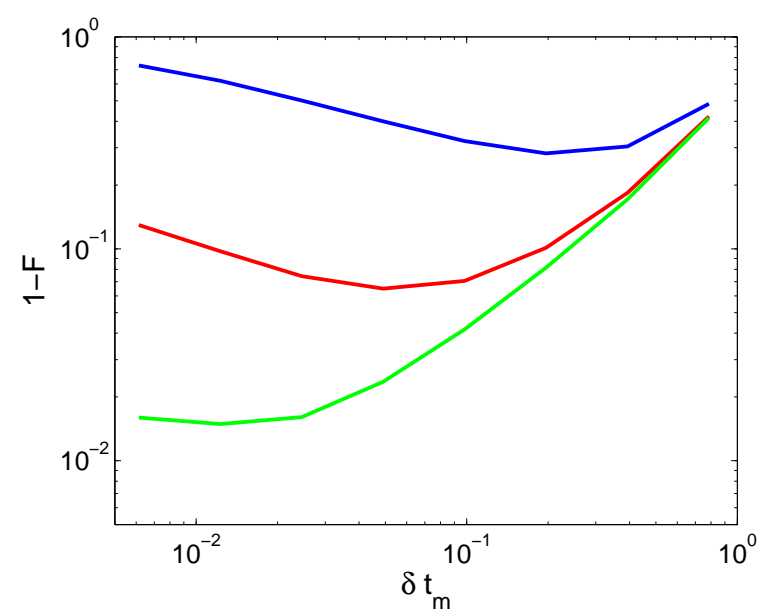

FIG. 7: (color online) Error of three-qubit gate $\exp (-i \pi / 4 H)$ with $H_{2}=\sigma_{z}^{(1)} \sigma_{z}^{(2)} \sigma_{z}^{(3)}+\sum_{k=1}^{3} \sigma_{x}^{(k)}$ generated by sequential applications of $\exp \left(-i \delta t_{m} \sigma_{z}^{(1)} \sigma_{z}^{(2)} \sigma_{z}^{(3)}\right)$ and $\exp \left(-i \delta t_{m} \sum_{k=1}^{3} \sigma_{x}^{(k)}\right)$. The three-qubit interaction is generated by the commutator method, and the figure shows the derivation from ideal gate as measured by the fidelity, $1-F$, plotted against different values of $\delta t_{m}$. Curves from top to bottom correspond to $p=0.9$ (blue), $p=0.99$ (red), $p=0.999$ (green), where $p=\exp (-\pi \kappa)$. Notice that there is an optimal choice for $\delta t_{m}$ to maximize the gate fidelity (see main text for details).

\section{E. Simulation of quDits using qudits}

We have illustrated the commutator method for qubits, however the results can without further effort be directly adopted to quDits. When using pairwise interacting $d-$ level systems to simulate an arbitrary Hamiltonian of a single $D$-level system with $D>d$, an effective $m$-body interaction Hamiltonian is required, where

$$
m=\left\lceil\frac{\log D}{\log d}\right\rceil,
$$


and $\lceil x\rceil$ denotes the next largest integer. This immediately follows from the fact that the Hilbert space of $m$ interacting qudits has dimension $d^{m}$, and $D \geq d^{m}$ is required that the $m$ qudits can simulate a single quDit, i.e. a $D$-dimensional system. The increase of noise level (dilation factor), the LNE and the fidelity are given by exactly the same expressions as in the case of qubits (see Eqs. 147/161), where the definition of LNE has to be adopted accordingly (local white noise of individual qudits rather than qubits).

Similarly, the simulation of pairwise interacting $D$ level systems requires $m$-body interactions of qudits, where

$$
m=\left\lceil 2 \frac{\log D}{\log d}\right\rceil .
$$

The simulation of many-body interactions of quDits, say $n$-body interactions, requires effective $m$-body interactions of qudits with $m=\lceil n \log D / \log d\rceil$.

\section{UNITARY CONJUGATION AND GRAPH STATE ENCODING}

We now turn to the second method to generate manybody Hamiltonians, unitary conjugation and graph state encoding (see Sec. III). In this case, certain two-qubit gates $U, U^{\dagger}$ (corresponding to a graph state encoding) are applied before and after the evolution with respect to a single- or two-body Hamiltonian, which leads to an effective evolution with respect to a many-body Hamiltonian. The choice of the encoding determines the manybody interaction that is generated. In this method, errors occur due to

(i) imperfect application of two qubit gates $U, U^{\dagger}$

(ii) imperfect application of single and two-body Hamiltonians.

While errors do not appear in higher order $\delta t^{\prime}$ as in the commutator method, they can still be significantly amplified. In particular, any simulation involves application of gates $U$ and $U^{\dagger}$ and hence any error associated with these gates. As these gates require the application of a basic Hamiltonian $H=\sigma_{z} \otimes \sigma_{z}$ for time $t=\pi / 4$, errors will be of order $\exp \left(-\kappa_{0} \pi / 4\right)$, even if $\delta t^{\prime} \ll 1$.

\section{A. Fixed graph state encoding}

We will first consider a fixed graph state encoding, i.e. the operations $U, U^{\dagger}$ only have to be applied at the beginning and end of simulation process. We start by analytically estimating the effect of noise in this case, and then consider exact numerical simulations which confirm our estimates.

\section{Three-body interaction}

The easiest way to generate a three-body interaction using a graph state encoding is to consider a three qubit system and an encoding operation

$$
U=U_{P G}^{(12)} U_{P G}^{(13)},
$$

where $U_{P G}=\operatorname{diag}(1,1,1,-1)$ is a phase gate. With this encoding, single qubit operations of the form $\exp \left(-i \delta t^{\prime} \sigma_{x}^{(1)}\right)$ allow one to generate an evolution with respect to the three-body Hamiltonian $H=\sigma_{x}^{(1)} \otimes \sigma_{z}^{(2)} \otimes$ $\sigma_{z}^{(3)}$ for time $t=\delta t^{\prime}$, i.e.

$$
U_{t}\left(\delta t^{\prime}\right)=U e^{-i \delta t^{\prime} \sigma_{x}^{(1)}} U^{\dagger}=e^{-i \delta t^{\prime} \sigma_{x}^{(1)} \otimes \sigma_{z}^{(2)} \otimes \sigma_{z}^{(3)}} .
$$

Phase noise: We now turn to a simple example to illustrate how noise effects this process. We consider a basic evolution that is governed by the Hamiltonian $H=\sigma_{z} \otimes \sigma_{z}$, and where the noise part is described by a Liouvillian that corresponds to single qubit dephasing, i.e. is given by Eq. 148. Since noise part and Hamiltonian part commute, the solution of the corresponding master equation is given by

$$
\rho(t)=\mathcal{D}^{(1)}(p) \mathcal{D}^{(2)}(p) U \rho U^{\dagger},
$$

where $U=\exp \left(-i t \sigma_{z}^{(1)} \otimes \sigma_{z}^{(2)}\right)$ and $\mathcal{D}_{t}^{(k)}$ is a dephasing map with parameter $p=p(t)=\exp \left(-\gamma_{0} t\right)$ (see Eq. 104). In the remainder of this section we will drop the $t$ subscript from the dephasing and depolarizing maps, so, unless otherwise stated, $\mathcal{D}_{t}^{(k)} \equiv \mathcal{D}^{(k)}$. Applying this noisy evolution for time $t=\pi / 4$, together with local rotations among the $z$-axis (i.e. operations of the form $\exp \left(-\delta t \sigma_{z}\right)$, a noisy phase gate can be generated

$$
\mathcal{E}_{P G} \rho=\mathcal{D}^{(1)}(p) \mathcal{D}^{(2)}(p) U_{P G} \rho U_{P G}^{\dagger},
$$

where $p=p(t)=\exp \left(-\gamma_{0} \pi / 4\right)$. The the total (noisy) process to generate the evolution with respect to the three-body Hamiltonian $H$ for time $t=\delta t^{\prime}$ is thus given by

$$
\mathcal{E} \rho=\mathcal{E}_{P G}^{(12)} \mathcal{E}_{P G}^{(13)} \hat{U}_{x}^{(1)}\left(\delta t^{\prime}\right) \mathcal{E}_{P G}^{(12)} \mathcal{E}_{P G}^{(13)} \rho,
$$

where $\hat{U}_{x}^{(1)}\left(\delta t^{\prime}\right) \rho=U_{x}\left(\delta t^{\prime}\right) \rho U_{x}\left(\delta t^{\prime}\right)^{\dagger}$ with $U_{x}\left(\delta t^{\prime}\right)=$ $\exp \left(-i \delta t^{\prime} \sigma_{x}\right)$. Using that the noise process commutes with $\sigma_{z}$, and that $\mathcal{D}(p) \mathcal{D}(p)=\mathcal{D}\left(p^{2}\right)$, one can simplify this expression and obtains

$$
\mathcal{E} \rho=\mathcal{D}^{(1)}\left(p^{2}\right) \hat{U}_{t}\left(\delta t^{\prime}\right) \mathcal{D}^{(1)}\left(p^{2}\right) \mathcal{D}^{(2)}\left(p^{2}\right) \mathcal{D}^{(3)}\left(p^{2}\right) \rho,
$$

where $U_{t}\left(\delta t^{\prime}\right)$ is given in Eq. 167. Notice that $\mathcal{D}^{(1)}$ and $U_{t}\left(\delta t^{\prime}\right)$ do not commute. Using Eq. 171 and the chaining inequality (Eq. 87) we can derive an exact bound on the Jamiołkowski distance and the corresponding fidelity. The distance between $\mathcal{E}$ and $\hat{U}_{t}\left(\delta t^{\prime}\right)$ can be expressed as,

$$
\begin{aligned}
& D\left(\mathcal{E}, \hat{U}_{t}\left(\delta t^{\prime}\right)\right) \\
& =D\left(\mathcal{D}^{(1)}\left(p^{2}\right) \hat{U}_{t}\left(\delta t^{\prime}\right) \mathcal{D}^{(1)}\left(p^{2}\right) \mathcal{D}^{(2)}\left(p^{2}\right) \mathcal{D}^{(3)}\left(p^{2}\right), \hat{U}_{t}\left(\delta t^{\prime}\right)\right)
\end{aligned}
$$


Applying the chaining property of the Jamiołkowski distance we find,

$$
\begin{aligned}
& D\left(\mathcal{E}, \hat{U}_{t}\left(\delta t^{\prime}\right)\right) \\
& \leq D\left(\mathcal{D}^{(1)}\left(p^{2}\right) \hat{U}_{t}\left(\delta t^{\prime}\right), \hat{U}_{t}\left(\delta t^{\prime}\right)\right) \\
& +D\left(\mathcal{D}^{(1)}\left(p^{2}\right) \mathcal{D}^{(2)}\left(p^{2}\right) \mathcal{D}^{(3)}\left(p^{2}\right), \mathbb{1}\right) \\
& =D\left(\mathcal{D}^{(1)}\left(p^{2}\right), \mathbb{1}\right)+D\left(\mathcal{D}^{(1)}\left(p^{2}\right) \mathcal{D}^{(2)}\left(p^{2}\right) \mathcal{D}^{(3)}\left(p^{2}\right), \mathbb{1}\right)
\end{aligned}
$$

Noting the fidelity of a one qubit dephasing channel with the identity is easily calculated and one finds that,

$$
F\left(\mathcal{D}^{(1)}\left(p^{2}\right), \mathbb{1}\right)=\frac{1+p^{2}}{2}
$$

and

$$
F\left(\mathcal{D}^{(1)}\left(p^{2}\right) \mathcal{D}^{(2)}\left(p^{2}\right) \mathcal{D}^{(3)}\left(p^{2}\right), \mathbb{1}\right)=\left(\frac{1+p^{2}}{2}\right)^{3}
$$

Which yields the corresponding distances,

$$
\begin{gathered}
D\left(\mathcal{D}^{(1)}\left(p^{2}\right), \mathbb{1}\right)=\sqrt{1-\frac{1+p^{2}}{2}} \\
D\left(\mathcal{D}^{(1)}\left(p^{2}\right) \mathcal{D}^{(2)}\left(p^{2}\right) \mathcal{D}^{(3)}\left(p^{2}\right), \mathbb{1}\right)=\sqrt{1-\left(\frac{1+p^{2}}{2}\right)^{3}}
\end{gathered}
$$

Thus, $D\left(\mathcal{E}, \hat{U}_{t}\left(\delta t^{\prime}\right)\right)$ has the upper bound,

$$
\begin{aligned}
& D\left(\mathcal{E}, \hat{U}_{t}\left(\delta t^{\prime}\right)\right) \\
& \leq \sqrt{1-\frac{1+p^{2}}{2}}+\sqrt{1-\left(\frac{1+p^{2}}{2}\right)^{3}}
\end{aligned}
$$

which provides an lower bound on the fidelity, $D\left(\mathcal{E}, \hat{U}_{t}\left(\delta t^{\prime}\right)\right)$ by

$$
\begin{aligned}
& F\left(\mathcal{E}, \hat{U}_{t}\left(\delta t^{\prime}\right)\right) \geq 1- \\
& \left(\sqrt{1-\frac{1+p^{2}}{2}}+\sqrt{1-\left(\frac{1+p^{2}}{2}\right)^{3}}\right)^{2}
\end{aligned}
$$

For small $\delta t^{\prime}$ a much simpler expression for the fidelity can be found that is tighter than the above bound. Using that $\mathcal{D}(p) \hat{U}_{x}\left(\delta t^{\prime}\right)=\hat{U}_{x}\left(\delta t^{\prime}\right) \tilde{\mathcal{D}}(p)$, where

$$
\tilde{\mathcal{D}}(p) \rho=p \rho+\frac{1-p}{2}\left(\rho+A \rho A^{\dagger}\right)
$$

and $A=\hat{U}_{t}\left(\delta t^{\prime}\right)^{\dagger} \sigma_{z} \hat{U}_{t}\left(\delta t^{\prime}\right)$. It follows that the total process can also be written as,

$$
\mathcal{E} \rho=\hat{U}_{t}\left(\delta t^{\prime}\right) \tilde{\mathcal{D}}^{(1)}\left(p^{2}\right) \mathcal{D}^{(1)}\left(p^{2}\right) \mathcal{D}^{(2)}\left(p^{2}\right) \mathcal{D}^{(3)}\left(p^{2}\right) \rho,(181)
$$

i.e. a sequence of certain local noise processes followed by the ideal evolution. The Jamiołkowski fidelity of the total map $\mathcal{E}$ with respect to the desired evolution $U_{t}\left(\delta t^{\prime}\right)$, $F\left(\mathcal{E}, U_{t}\left(\delta t^{\prime}\right)\right)$ is thus the same as the one of the map $\tilde{\mathcal{E}} \rho=\tilde{\mathcal{D}}^{(1)}\left(p^{2}\right) \mathcal{D}^{(1)}\left(p^{2}\right) \mathcal{D}^{(2)}\left(p^{2}\right) \mathcal{D}^{(3)}\left(p^{2}\right) \rho$ with respect to the identity, $F(\tilde{\mathcal{E}}, \mathbb{1})$. For small $\delta t^{\prime}$, one has that $\tilde{\mathcal{D}} \approx \mathcal{D}$, and hence

$$
\tilde{\mathcal{E}} \rho \approx \mathcal{D}^{(1)}\left(p^{4}\right) \mathcal{D}^{(2)}\left(p^{2}\right) \mathcal{D}^{(3)}\left(p^{2}\right) \rho .
$$

The fidelity $F(\tilde{\mathcal{E}}, \mathbb{1})$, and hence $F\left(\mathcal{E}, U_{t}\left(\delta t^{\prime}\right)\right)$ can be easily calculated, and one obtains

$$
\begin{aligned}
F\left(\mathcal{E}, U_{t}\left(\delta t^{\prime}\right)\right) & \approx\left(\frac{1+p^{4}}{2}\right)\left(\frac{1+p^{2}}{2}\right)^{2} \\
& \approx 1-\pi \gamma_{0},
\end{aligned}
$$

where $p=\exp \left(-\gamma_{0} \pi / 4\right)$ and the last simplification only holds for $\gamma_{0} \pi / 4 \ll 1$. Under the same approximation the exact lower bound on the fidelity that we found in Eq. 179 can be approximated to

$$
F\left(\mathcal{E}, U_{t}\left(\delta t^{\prime}\right)\right) \gtrsim 1-\left(1+\frac{\sqrt{3}}{2}\right) \pi \gamma_{0},
$$

demonstrating that our bound provides a relatively good approximation to the achievable fidelity in this parameter regime.

The local noise equivalent (see Sec. VIA for definition) can be evaluated under the previous assumptions. We find that $F=\left(\frac{1+\exp \left(-\delta t^{\prime} \gamma\right)}{2}\right)^{3} \approx 1-\frac{3}{2} \delta t^{\prime} \gamma$, and hence the local noise equivalent $\gamma$ is given by

$$
\gamma \approx \frac{2 \pi}{3 \delta t^{\prime}} \gamma_{0}
$$

White noise: Similar arguments can be used to estimate the effect of white noise, i.e. when the Liouvillian describing the noise process in the basic two-qubit interaction is a sum of local terms described by Eq. 103 with $s=1 / 2$ and $B=C \equiv \kappa_{0}$. The solution of this master equation (when taking interaction into account) does in general not lead a simple form such as Eq. 186, where the ideal evolution is simply composed by noise channels. However, one may still use such a form, and still obtains a good approximation, in particular if $\kappa_{0}$ is relatively small.

In particular, we have

$$
\rho(t) \approx \mathcal{M}^{(1)}(p) \mathcal{M}^{(2)}(p) U \rho U^{\dagger},
$$

where $U=\exp \left(-i t \sigma_{z}^{(1)} \otimes \sigma_{z}^{(2)}\right)$ and $\mathcal{M}$ is a depolarizing map with parameter $p=p(t)=\exp \left(-\kappa_{0} t\right.$ ) (see Eq. 105). As the depolarizing channel does not commute with $U$ the best analytic bound that we can find on the Jamiołkowski distance is that given by the simple bound derived in Eq. 95. Noting that,

$$
\begin{aligned}
& F\left(\mathcal{M}^{(1)}(p) \mathcal{M}^{(2)}(p) U, U\right)=F\left(\mathcal{M}^{(1)}(p) \mathcal{M}^{(2)}(p), \mathbb{1}\right) \\
& =\left(\frac{1+3 p}{4}\right)^{2}
\end{aligned}
$$


and that in order to simulate $U_{t}\left(\delta t^{\prime}\right)$ we must use $U$ four times we find,

$$
D\left(\mathcal{E}, U_{t}\left(\delta t^{\prime}\right)\right) \leq 4 \sqrt{1-\left(\frac{1+3 p}{4}\right)^{2}} .
$$

Thus, the corresponding Jamiołkowski fidelity is lower bounded by:

$$
F\left(\mathcal{E}, U_{t}\left(\delta t^{\prime}\right)\right) \geq 1-16\left(1-\left(\frac{1+3 p}{4}\right)^{2}\right)
$$

Following the same line of argument as for the dephasing calculation above, we can find a simple approximation that beats this bound when $\delta t^{\prime}$ is small. One finds that the total (noisy) process is approximately described by

$$
\tilde{\mathcal{E}} \rho \approx \mathcal{M}^{(1)}\left(p^{4}\right) \mathcal{M}^{(2)}\left(p^{2}\right) \mathcal{M}^{(3)}\left(p^{2}\right) \rho .
$$

This leads to a final fidelity of the total process given by

$$
\begin{aligned}
F\left(\mathcal{E}, U_{t}\left(\delta t^{\prime}\right)\right) & \approx\left(\frac{1+3 p^{4}}{4}\right)\left(\frac{1+3 p^{2}}{4}\right)^{2} \\
& \approx 1-\frac{3 \pi}{2} \kappa_{0}
\end{aligned}
$$

where here $p=\exp \left(-\kappa_{0} \pi / 4\right)$ and the last simplification only holds for $\kappa_{0} \pi / 4 \ll 1$. If we alternately used the exact error bound of Eq. 189 in the same parameter regime we find

$$
F\left(\mathcal{E}, U_{t}\left(\delta t^{\prime}\right)\right) \gtrsim 1-3 \pi \kappa_{0}
$$

which is close to the approximation of Eq. 191.

Again, the local noise equivalent can be evaluated, where in the case of depolarizing noise one can estimate $F \approx\left(\frac{1+3 \exp \left(-\kappa \delta t^{\prime}\right)}{4}\right)^{3} \approx 1-\frac{9}{4} \delta t^{\prime} \kappa$, which leads to a local noise equivalent

$$
\kappa \approx \frac{2 \pi}{3 \delta t^{\prime}} \kappa_{0}
$$

Notice in particular that the final expression for the fidelity is independent of $\delta t^{\prime}$, the time for which the threebody interaction should be applied. Such a constant fidelity in turn implies that the local noise equivalent grows with decreasing $\delta t^{\prime}$. This can easily be understood by recalling that the reliability parameter $p$ for dephasing or depolarizing noise decreases with time, and hence a constant fidelity actually means a larger noise level for short time evolutions.

These observations are in contrast to the commutator method, where the fidelity $F$ essentially decreases for larger $\delta t^{\prime}$. A direct comparison shows that for small values of $\delta t^{\prime}$, the commutator method leads much better fidelities. However, when considering evolutions for longer times $\delta t^{\prime}$, e.g. the generation of gates or the simulation of the evolution with respect to a more complicated Hamiltonian generated by Hamiltonian simulation techniques, the graph state encoding method performs significantly better (see table I). In particular when considering time evolution over several full cycles (multiples of $2 \pi$ ), the advantage of graph state encoding method becomes obvious. In this case, the local noise equivalent can even be smaller than $\kappa_{0}$. The latter processes appear, for instance, when simulating an evolution with respect to a time dependent Hamiltonian in such a way that adiabatic passage to the ground state of the final Hamiltonian occurs, and hence the ground state of this Hamiltonian is generated [14, 16].

We remark that in our estimation of the total fidelity we have assumed small values $\delta t^{\prime}$. It turns out, however, that even when taking all errors into account and performing numerical simulations of the total process, there is essentially no dependence on $\delta t^{\prime}$. In addition, our estimates turn out to be very accurate (see table I). This table shows the resulting fidelities when using graph state encoding techniques as described above to generate unitary evolutions with respect to Hamiltonian (i) $H_{1}=\sigma_{z}^{(1)} \sigma_{z}^{(2)} \sigma_{z}^{(3)}$ for time $t=\pi / 4$. In this case, the graph state encoding $G_{1}$ corresponds to $U=U_{P G}^{(12)} U_{P G}^{(13)}$ and single-qubit operations of the form $\exp \left(-i \delta t^{\prime} \sigma_{x}^{(1)}\right)$ are used to generate the desired three-body Hamiltonian. Also the usage of an alternative graph state encoding $G_{2}$ using only $U=U_{P G}^{(12)}$ together with the application of two-qubit operators $\exp \left(-i \delta t^{\prime} \sigma_{x}^{(2)} \sigma_{z}^{(3)}\right)$ to generate the desired three-qubit Hamiltonian is shown.

The latter graph state encoding $G_{2}$ is also used to generate time evolution with respect to the Hamiltonian (ii) $H_{2}=\sigma_{z}^{(1)} \sigma_{z}^{(2)} \sigma_{z}^{(3)}+\sum_{k=1}^{3} \sigma_{x}^{(k)}$ for time $t=\pi / 4$. In this case, Hamiltonian simulation techniques need to be applied to generate evolutions with respect to the noncommuting terms $\sigma_{z}^{(1)} \sigma_{z}^{(2)} \sigma_{z}^{(3)}$ and $\sum_{k=1}^{3} \sigma_{x}^{(k)}$ for short times $\delta t_{m}$ sequentially. Up to an additional local basis change (a Hadamard operation Had on qubit 2), the first term is generated by the two-qubit Hamiltonian $H_{1}^{\prime}=\sigma_{x}^{(2)} \sigma_{z}^{(3)}$, while the second term is produced from the Hamiltonian $H_{2}^{\prime}=\sigma_{x}^{(1)} \sigma_{z}^{(2)}+\sigma_{z}^{(2)}+\sigma_{x}^{(3)}$. The total procedure thus involves the application of $U_{P G}^{(12)} \mathrm{Had}^{(2)}$, followed by sequential application of $H_{1}^{\prime}, H_{2}^{\prime}$ for short times $\delta t_{m}$ a total of $(\pi / 4) / \delta t_{m}$ times, and a final application of $\operatorname{Had}^{(2)} U_{P G}^{(12)}$. Notice that the same examples are considered in the case of the commutator method in Sec. VIIID

\section{Many-body interaction}

It is straightforward to obtain methods to generate a $m$-body Hamiltonian using graph state encoding, and analytic estimates for the influence of noise. In particular, the graph state encoding

$$
U=\prod_{k=2}^{m} U_{P G}^{(1 k)}
$$




\begin{tabular}{|c||c|c|c|c|c|}
\hline & $H_{1}$, anal. & $H_{1}, G_{1}$ & $H_{1}, G_{2}$ & $H_{1}, C$ & $H_{2}, G_{2}$ \\
\hline \hline $\mathrm{p}=0.9$ & $\mathrm{~F}=0.8552$ & $\mathrm{~F}=0.8545$ & $\mathrm{~F}=0.8887$ & $\mathrm{~F}=0.7062$ & $\mathrm{~F}=0.8545$ \\
\hline $\mathrm{p}=0.99$ & $\mathrm{~F}=0.9851$ & $\mathrm{~F}=0.9850$ & $\mathrm{~F}=0.9888$ & $\mathrm{~F}=0.9305$ & $\mathrm{~F}=0.9850$ \\
\hline $\mathrm{p}=0.999$ & $\mathrm{~F}=0.9985$ & $\mathrm{~F}=0.9985$ & $\mathrm{~F}=0.9989$ & $\mathrm{~F}=0.9841$ & $\mathrm{~F}=0.9985$ \\
\hline
\end{tabular}

TABLE I: Table shows fidelities to generate gate $U_{1}=$ $\exp \left(-i \pi / 4 H_{1}\right)$ using graph state encoding (column 1: analytic estimate using graph state encoding $G_{1}$, columns two and three: numerical simulation using graph state encoding $G_{1}$ and $G_{2}$ respectively) or commutator method with optimal time $\delta t_{m}$ (column four). The last column gives the fidelity to generate the time evolution $U_{2}=\exp \left(-i \pi / 4 H_{2}\right)$ using graph state encoding $G_{2}$ (see text for details). A white noise parameter $p=\exp \left(-\kappa_{0} \pi\right)$ is assumed that corresponds to a coupling strength $\kappa_{0}$ to the thermal bath that would lead to depolarizing map with parameter $p$ when applied for time $t=\pi$. Notice that this corresponds to $p^{\prime}=p^{1 / 4}$ when using the formula for analytic estimate of fidelity, Eq. 191 where noisy evolution is only applied for time $t=\pi / 4$.

together with the evolution with respect to the singlequbit Hamiltonian $H=\sigma_{x}^{(1)}$ for time $t=\delta t^{\prime}$ allows one to generate the $m$-qubit gate $\exp \left(-i \delta t^{\prime} \otimes_{k=2}^{m} \sigma_{z}^{(k)} \otimes \sigma_{x}^{(1)}\right)$, i.e.

$$
U \exp \left(-i \delta t^{\prime} \sigma_{x}^{(1)}\right) U^{\dagger}=\exp \left[-i \delta t^{\prime}\left(\otimes_{k=2}^{m} \sigma_{z}^{(k)}\right) \otimes \sigma_{x}^{(1)}\right\}(195)
$$

Following precisely the same line of reasoning as in the case of three-qubit interaction, $m=3$, one obtains in the case of phase noise that the total noisy evolution is described by (compare with Eq. 181)

$$
\mathcal{E} \rho=\hat{U}_{t}\left(\delta t^{\prime}\right) \tilde{\mathcal{D}}^{(1)}\left(p^{m-1}\right) \mathcal{D}^{(1)}\left(p^{m-1}\right) \prod_{k=2}^{m} \mathcal{D}^{(k)}\left(p^{2}\right) \rho .(196)
$$

We find that the analytic bound on the fidelity of this operation given to us by the chaining property of the Jamiołkowski distance to be,

$$
\begin{aligned}
& F\left(\mathcal{E}, \hat{U}_{t}\left(\delta t^{\prime}\right)\right) \geq 1- \\
& \left(\sqrt{1-\frac{1+p^{m-1}}{2}}+\sqrt{1-\frac{1+p^{m-1}}{2}\left(\frac{1+p^{2}}{2}\right)^{m-1}}\right)^{2} .
\end{aligned}
$$

When $\delta t^{\prime}$ is small a simple estimation of fidelity can be made

$$
\begin{aligned}
F\left(\mathcal{E}, U_{t}\left(\delta t^{\prime}\right)\right) & \approx\left(\frac{1+p^{2(m-1)}}{2}\right)\left(\frac{1+p^{2}}{2}\right)^{m-1} \\
& \approx 1-2(m-1) \frac{\pi}{4} \gamma_{0}
\end{aligned}
$$

where $p=\exp \left(-\gamma_{0} \pi / 4\right)$ and the last simplification is valid for $\pi / 4 \gamma_{0} \ll 1$. For the local noise equivalent we find $\gamma \approx \frac{(m-1) \pi}{m \delta t^{\prime}} \gamma_{0}$.
Similarly, in the case of depolarizing (or white) noise, one finds that the fidelity can be lower bounded by

$$
F\left(\mathcal{E}, U_{t}\left(\delta t^{\prime}\right)\right) \geq 1-(m-1)^{2}\left(1-\left(\frac{1+3 p}{4}\right)^{2}\right) .
$$

We can also directly evaluate the fidelity in the small $\delta t^{\prime}$ limit to be

$$
\begin{aligned}
F\left(\mathcal{E}, U_{t}\left(\delta t^{\prime}\right)\right) & \approx\left(\frac{1+3 p^{2(m-1)}}{4}\right)\left(\frac{1+3 p^{2}}{4}\right)^{m-1} \\
& \approx 1-3(m-1) \frac{\pi}{4} \kappa_{0}
\end{aligned}
$$

where here $p=\exp \left(-\kappa_{0} \pi / 4\right)$ and the last simplification only holds for $\kappa_{0} \pi / 4 \ll 1$. Again, the final expressions are independent of the total time $t=\delta t^{\prime}$. The local noise equivalent can be determined to be $\kappa \approx \frac{(m-1) \pi}{m \delta t^{\prime}} \kappa_{0}$.

\section{Timing errors}

In this subsection we consider the effect of timing errors on the fixed graph state encoding method. Recall that in Subsection [VII] we introduced the idea of a random timing error. Such an error arises due to our inability to apply any Hamiltonian for an absolutely precise amount of time.

Consider the 3-qubit simulation protocol of the previous subsection as given by Eq. 167

$$
\begin{aligned}
U_{t}\left(\delta t^{\prime}\right) & =U_{P G}^{(12)} U_{P G}^{(13)} e^{-i \delta t^{\prime} \sigma_{x}^{(1)}} U_{P G}^{(12)} U_{P G}^{(13)} \\
& =e^{-i \delta t^{\prime} \sigma_{x}^{(1)} \otimes \sigma_{z}^{(2)} \otimes \sigma_{z}^{(3)}} .
\end{aligned}
$$

Each of the phase gates, $U_{P G}$, can be generated by evolving a two qubit Ising interaction Hamiltonian, $H=$ $\sigma_{z} \otimes \sigma_{z}$, for a time $t=\pi / 4$ and applying single qubit $\sigma_{z}$ rotation to each qubit. If we have a timing error on an Ising Hamiltonian that generates a phase gate, then we can represent this noisy process by the following CP map

$$
\mathcal{E}_{P G}(\rho)=\mathcal{T} U_{P G} \rho U_{P G}
$$

where $\mathcal{T}$ is the CP map given in Eq. 122, The map $\mathcal{T}$ is a correlated two-qubit dephasing channel. The noisy version this simulation protocol is expressed by

$$
\mathcal{E}(\rho)=\mathcal{T}^{(12)} \mathcal{T}^{(13)} \hat{U}_{t}\left(\delta t^{\prime}\right) \mathcal{T}^{(12)} \mathcal{T}^{(13)}(\rho) .
$$

using the chaining property of the Jamiołkowski distance it is possible to bound the fidelity of this operation. Noting that neither $\mathcal{T}_{12}$ nor $\mathcal{T}_{13}$ commute with $\hat{U}_{t}\left(\delta t^{\prime}\right)$ and applying the chaining inequality we find,

$$
D\left(\mathcal{E}, U_{t}\left(\delta t^{\prime}\right)\right) \leq 2 D\left(\mathcal{T}^{(12)} \mathcal{T}^{(13)}, \mathbb{1}\right) .
$$

Assuming that the timing error was Gaussian distributed with a standard deviation of $\sigma$ (as was done in Subsection VII) we find

$$
F\left(\mathcal{T}^{(12)} \mathcal{T}^{(13)}, \mathbb{1}\right)=\left(\frac{1+q}{2}\right)^{2}
$$


where $q=e^{-2 \sigma^{2}}$. The resulting lower bound on the fidelity is,

$$
F\left(\mathcal{E}, U_{t}\left(\delta t^{\prime}\right)\right) \geq 1-4\left(1-\left(\frac{1+q}{2}\right)^{2}\right) .
$$

If we assume that the timing error is very small, that is $\sigma^{2} \ll 1$ then we can find a simpler form for this bound. Noting that for small $\sigma^{2}$

$$
F\left(\mathcal{T}^{(12)} \mathcal{T}^{(13)}, \mathbb{1}\right) \approx 1-2 \sigma^{2},
$$

then it follows that

$$
F\left(\mathcal{E}, U_{t}\left(\delta t^{\prime}\right)\right) \gtrsim 1-8 \sigma^{2} .
$$

The above equations can be simply generalized to the $m$ qubit case. Recall that in the $m$-qubit case that we must apply the following encoding operation,

$$
U_{e}=\prod_{k=2}^{m} U_{P G}^{(1 k)} .
$$

The result of timing errors on the phase gates in $U_{e}$ is given by the following map,

$$
\mathcal{E}_{e}(\rho)=\prod_{k=2}^{m} \mathcal{T}^{(1 k)} U_{e} \rho U_{e}
$$

The fidelity, $F\left(\mathcal{E}_{e}, U_{e}\right)=F\left(\prod_{k=2}^{m} \mathcal{T}^{(1 k)}, \mathbb{1}\right)$, and is given by:

$$
F\left(\mathcal{E}_{e}, U_{e}\right)=F\left(\prod_{k=2}^{m} \mathcal{T}^{(1 k)}, \mathbb{1}\right)=\left(\frac{1+q}{2}\right)^{m-1} .
$$

This results in the following error bound for the entire simulation protocol,

$$
\begin{aligned}
F\left(\mathcal{E}, U_{t}\left(\delta t^{\prime}\right)\right) & \geq 1-4\left(1-F\left(\mathcal{E}_{e}, U_{e}\right)\right) \\
& =1-4\left(1-\left(\frac{1+q}{2}\right)^{m-1}\right) .
\end{aligned}
$$

When $\sigma^{2} \ll 1$ the fidelity of performing an encoding operation is given by,

$$
F\left(\mathcal{E}_{e}, U_{e}\right)=F\left(\prod_{k=2}^{m} \mathcal{T}^{(1 k)}, \mathbb{1}\right) \approx 1-(m-1) \sigma^{2}
$$

which results in the following bound on the entire simulation protocol,

$$
F\left(\mathcal{E}, U_{t}\left(\delta t^{\prime}\right)\right) \gtrsim 1-4(m-1) \sigma^{2} .
$$

So far we have considered the situation where the operation $U_{P G}^{(12)} U_{P G}^{(13)}$ is generated by single qubit $\sigma_{z}$ rotations and evolutions of the Hamiltonians $H^{(12)}=\sigma_{z}^{(1)} \sigma_{z}^{(2)}$ and $H^{(13)}=\sigma_{z}^{(1)} \sigma_{z}^{(3)}$. We could have in principle generated the product of these phase gates by applying single-qubit $\sigma_{z}$ rotations and evolving by the collective Hamiltonian $H^{(123)}=H^{(12)}+H^{(13)}$. Such an evolution would give rise to the following $\mathrm{CP}$ map,

$$
\mathcal{E}^{(123)}(\rho)=\mathcal{T}^{(123)} U_{P G}^{(12)} U_{P G}^{(13)} \rho U_{P G}^{(12)} U_{P G}^{(13)} .
$$

Where the CP map $\mathcal{T}^{(123)}$ is a result of timing error on the Hamiltonian $H_{123}$. The form of $\mathcal{T}^{(123)}$ was derived in Subsection VII for the case where the timing error is Gaussian distributed with a standard deviation of $\sigma$ (this is given in Eq. 130). Noting that

$$
\begin{aligned}
F\left(\mathcal{E}^{(123)}, U_{P G}^{(12)} U_{P G}^{(13)}\right) & =F\left(\mathcal{E}^{(123)}, e^{-i \frac{\pi}{4} H^{(123)}}\right) \\
& =F\left(\mathcal{T}^{(123)}, \mathbb{1}\right)
\end{aligned}
$$

the fidelity of this channel was derived and we found it to be

$$
F\left(\mathcal{T}^{(123)}, \mathbb{1}\right)=\frac{3}{2^{3}}+\frac{1}{2} e^{-2 \sigma^{2}}+\frac{1}{2^{3}}\left(e^{-2 \sigma^{2}}\right)^{4} .
$$

Using similar methods to that which we have seen above, we can calculate a bound on the fidelity $F\left(\mathcal{E}, U_{t}\left(\delta t^{\prime}\right)\right)$ given that we used a collective Ising Hamiltonian $H^{(123)}$ as our entangling resource,

$$
F\left(\mathcal{E}, U_{t}\left(\delta t^{\prime}\right)\right) \geq 1-4\left(1-F\left(\mathcal{T}^{(123)}, \mathbb{1}\right)\right) .
$$

When $\sigma^{2} \ll 1$, we see that

$$
F\left(\mathcal{T}^{(123)}, \mathbb{1}\right) \approx 1-2 \sigma^{2}
$$

which results in a lower bound for $F\left(\mathcal{E}, U_{t}\left(\delta t^{\prime}\right)\right)$,

$$
F\left(\mathcal{E}, U_{t}\left(\delta t^{\prime}\right)\right) \gtrsim 1-8 \sigma^{2} .
$$

Interestingly, we see that this is the same fidelity that we derived in the case where our entangling operations were generated by two-qubit Hamiltonian interactions.

Generalizing to the $m$-qubit case, we recall that we must apply an $m$-qubit encoding operation, $U_{e}=$ $\prod_{k=2}^{m} U_{P G}^{(1 k)}$. This $m$-qubit encoding operation can be performed by single qubit $\sigma_{z}$ operations and an evolution for a time $\pi / 4$ of the Hamiltonian,

$$
H_{e}=\sum_{k=2}^{m} \sigma_{z}^{(1)} \sigma_{z}^{(k)} .
$$

$H_{e}$ is a sum of commuting unitary operations, in Subsection VII 2 we analyzed the effect of timing errors on such Hamiltonians. Because there are only $m-1 \sigma_{z}^{(1)} \sigma_{z}^{(k)}$ terms in $H_{e}$, and because of the geometry of the interaction graph, it is possible to find an analytic expression for a timing error in the application of the Hamiltonian $H_{e}$. We found the resulting fidelity to be,

$$
\begin{aligned}
& F\left(\mathcal{E}_{e}, U_{e}\right)=F\left(\mathcal{T}_{e}, \mathbb{1}\right) \\
& =\frac{1}{2^{2 n}}\left(\begin{array}{c}
2 n \\
n
\end{array}\right)+\frac{1}{2^{2 n-1}} \sum_{k=0}^{n-1}\left(\begin{array}{c}
2 n \\
k
\end{array}\right) e^{-2(n-k)^{2} \sigma^{2}},
\end{aligned}
$$


where $n=m-1$. As we saw above, this allows us $\mathrm{t}$ bound the fidelity of the entire simulation protocol $t$ be:

$$
F\left(\mathcal{E}, U_{t}\left(\delta t^{\prime}\right)\right) \geq 1-4\left(1-F\left(\mathcal{T}^{(12 \ldots m)}, \mathbb{1}\right)\right) .
$$

All of the noise that appears in our analysis of th graph state encoding protocol appears within the tim ing error of the encoding operation, $U_{e}$. As we saw, th success of the protocol depends on the fidelity, $F\left(\mathcal{E}_{e}, U_{e}\right.$ The higher this fidelity, the higher the fidelity of the ove all protocol. Our analysis begs the obvious question; $i$ the presence of timing errors is it better to use a collectiv Ising Hamiltonian acting on many qubits or many appl cations of two-qubit Ising Hamiltonians? We addressed more general version of this question in SubsectionVII: Applying the results of Subsection VII 2 to the case c applying the encoding operation $U_{e}$ we find

$$
F\left(\prod_{k=2}^{m} \mathcal{T}^{(1 k)}, \mathbb{1}\right) \leq F\left(\mathcal{T}^{(12 \ldots m)}, \mathbb{1}\right),
$$

where we assuming that the standard deviation of the timing errors in the two-qubit Hamiltonians is the same as that for the collective Hamiltonian $H_{e}$. Under these assumptions using a collective Ising Hamiltonian results in a larger fidelity for the simulation. In Figure 8 we have plotted the fidelity of performing the encoding operation $U_{e}$ using a collective $m-q u b i t$ Ising Hamiltonian and using two-qubit Ising Hamiltonians. We see that for $\sigma \rightarrow$ 0 that both methods converge to give a fidelity of one. As $\sigma$ increases we see that the fidelity of the two methods diverges and that this effect becomes more pronounced as the number of qubits coupled by $U_{e}$ increases.

\section{B. Variable graph state encoding}

In our previous consideration, we have assumed that a fixed graph state encoding is sufficient to generate the desired many-body Hamiltonian using only singleand two-qubit interactions. In Sec. III we have discussed several examples where this is indeed the case, and the strength of the graph state encoding method is certainly in such situations. In particular, if the total time for which an evolution with respect to a many-body Hamiltonian should be generated is large, the graph state encoding method performs significantly better than the commutator method.

In certain situations, however, a fixed graph state encoding is not sufficient and the encoding has to be changed frequently. Any change of encoding requires the application of phase gates, i.e. applying the two-body interaction described by the Hamiltonain $H=\sigma_{z} \otimes \sigma_{z}$ for time $t=\pi / 4$. Such a situation is discussed in detail in Sec. III E 2, Recall that for a given interaction Hamiltonian, it can be that not all required (non-commuting) interaction terms $H_{1}, \ldots, H_{n}$ can be generated using a
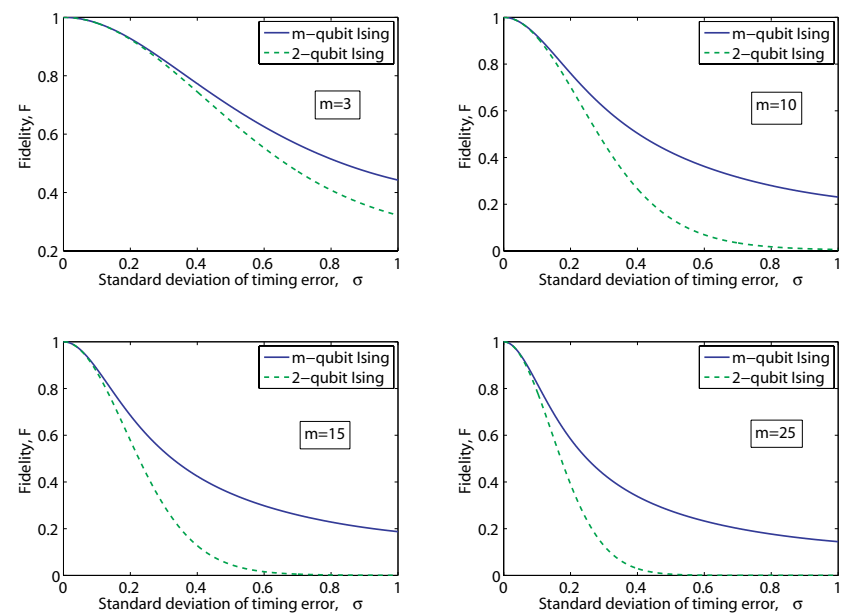

FIG. 8: (Color online) In these figure we see how the fidelity of performing the encoding operation $U_{e}$ changes with the standard deviation of a timing error, $\sigma$. We have examined how this fidelity changes with the number of qubits, $m$, encoded by $U_{e}$. The solid line represents the fidelity of performing the encoding operation with a single collective Ising Hamiltonian $H_{e}$. The dashed line represents the fidelity as a result of using a number of two-qubit Ising Hamiltonians $H_{1 k}$ to generate $U_{e}$. We see that as $\sigma \rightarrow 0$ the two methods give the same fidelity. However, it appears for larger $\sigma$ the collective method is superior. This effect becomes more apparent as $m$ increases.

fixed graph state encoding. Hence Hamiltonian simulation techniques need to be applied, requiring the sequential evolution with respect to Hamiltonians $H_{1}, \ldots, H_{n}$ for short times $\delta t^{\prime}$ that are generated with help of certain graph state encodings $G_{1}, G_{2}, \ldots, G_{n}$. As discussed in detail above, any change of graph state encoding introduces a certain (fixed) amount of noise. This is reflected in a constant fidelity of the simulation process using a fixed graph state encoding, or alternatively by an increase of the local noise equivalent that is essentially given by

$$
\kappa / \kappa_{0} \approx \pi / \delta t^{\prime}
$$

On the one hand, $\delta t^{\prime}$ needs to be chosen sufficiently small to ensure that errors due to taylor expansion in the simulation process remain small. On the other hand, smaller values of $\delta t^{\prime}$ lead a larger local noise equivalent due to frequent change of graph state encoding. Similar as in the commutator method, a compromise between these two competing requirements need to be chosen, such that for the simulation with respect to a certain Hamiltonian for time $t$ the resulting fidelity is maximized.

\section{TELEPORTATION BASED METHODS}

We now turn to teleportation based methods to generate many-body Hamiltonians. Recall that the basic idea 
is to use two-body interactions to generate certain entangled multi-party states $\left|\psi_{E}\right\rangle$. These states are then used as a resource to generate by means of (joint) measurements the desired evolution with respect to a manybody Hamiltonian for time $\delta t^{\prime}$. Depending on the chosen scheme, the states $\left|\psi_{E}\right\rangle$ are (i) weakly entangled and sequences of probabilistic teleportation processes together with preparation of states with increasing amount of entanglement are required to make the process deterministic; or (ii) maximally entangled and involve also an ancilla particle, which is used to chose the evolution time $\delta t^{\prime}$ and to make the process deterministic.

If two-body interactions are noisy, then the generated multi-party states are mixed. The fidelity of the state is determined by the quality of the two-body interaction. In turn, the fidelity of the multi-party states determines the noise level of the many-body interaction. We remark that for the approach (i), it is not always clear how to use two-body interactions for short times to generate the desired state. Even if this is possible, it turns out that approach (i) yields smaller fidelities than approach (ii). We will thus only consider approach (ii) in the following, and assume that maximally entangled states of several particles (including ancilla particles) are generated by twobody interactions, and processed by measurements. As in the case of graph state encoding, here we are again faced with a fixed amount of noise, independent of the desired interaction time $\delta t^{\prime}$. On the one hand, this results from the generation of the maximally entangled state $\left|\psi_{E}\right\rangle$, which involves application of two-body interactions for times $\delta t \approx O(\pi)$. On the other hand, the teleportation process consists of joint (Bell) measurements, which may also require the application of entangling operations that further increase the noise level.

In the following we will discuss a scenario where higher dimensional systems are used to simulate qubit systems (or more generally lower dimensional systems). Extra dimensions are used to store the particles corresponding to the state $\left|\psi_{E}\right\rangle$ and at the same time the system degrees of freedom which should be processed. In this case, the measurements in the teleportation process are local as they involve only single systems, and one can argue that the noise for such local processes is different (and lower) than for two-system interactions. Hence the dominating part of noise will come from the preparation of the entangled state $\left|\psi_{E}\right\rangle$. We will elaborate further on this scenario, and will also consider the case where additional auxiliary dimensions can be used to perform entanglement purification to reduce the amount of noise, or in the case when single system operations are perfect, to eliminate noise completely.

\section{A. Simulation of low dimensional systems using high dimensional systems}

In Sec. VIIIE we have argued that low dimensional systems of dimension $d$ can also be used to simulate in- teracting high dimensional systems of dimension $D$. In this case, many body Hamiltonians are required, even to achieve single-system operations on the simulated $D$ dimensional system. Here we consider the opposite approach, where high dimensional systems of dimension $D$ are used to simulate low dimensional systems of dimension $d$, and we concentrate on $d=2$, i.e. qubits. We will, however, not embed the qubits into the full Hilbert space of the $D$-dimensional systems, but rather associate each $D$-level system with a single qubit, i.e. we use only two of the $D$ dimensions to represent and store the quantum information. The remaining levels are used as auxiliary levels in such a way that operations on the the qubit systems can be performed in an simplified way, or to increase the fidelity of such operations and interactions.

To be more precise, we consider the case where the dimension $D$ is some power of two, $D=2^{m}$. In this case, the $D$-dimensional system can be considered as a system consisting of $m$ virtual subsystems (virtual qubits), where one of these qubits, the storage qubit $A_{1}$, is used for storage of quantum information, while the remaining $m-$ 1 qubits $A_{2}, \ldots, A_{m}$ are auxiliary systems. We remark that this simply corresponds to a labelling of the $D$ dimensional Hilbert space which we choose for notational convenience and to clearly distinguish between levels that are used for storage purposes and auxiliary levels. These virtual qubits do not have any true physical relevance.

Interactions between the auxiliary qubits and the storage qubit, as well as interactions between the auxiliary qubits, correspond to single system operations on the $D$ dimensional system. We also consider two-body interactions between two such $D$-dimensional systems labelled by $A$ and $B$. Depending on the type of interaction, such a two-body interaction can couple the two storage qubits $A_{1}$ and $B_{1}$, yielding a pairwise interaction, or two auxiliary qubits $A_{k}$ and $B_{k}$ (or combinations of these processes). We will assume that single system operations, which we also call local operations, can be performed with a high fidelity. The noise of such local processes is described by a noise parameter $p_{l}$ (when considering gates), or by coupling strengthes $\gamma_{l}, \kappa_{l}$ when considering single system interactions (i.e. interactions between auxiliary qubits and the storage qubit) that are described by a master equation. Interactions between different $D$ dimensional systems $A$ and $B$, which we also call nonlocal operations, can be performed with lower fidelity. We will denote the noise parameter by $p_{0}$, or the coupling strengthes by $\gamma_{0}, \kappa_{0}$. We remark that these assumptions are natural in the sense that often single system operations, i.e. interactions between different levels of the same system, are easier to perform than controlled interactions between two (spatially separated) systems. For example, atoms or ions have many potentially usable internal states (levels) which can be easily coupled via Ramanor Microwave transitions, while interactions between two such atoms or ions involves more complicated processes (e.g. induced dipole couplings, sequences of Laser pulses and couplings via motional states etc.). Note that a sim- 
ilar approach was considered in the context of quantum computation in Ref. [59], where the auxiliary levels where used to prepare entangled states shared between systems, which where then used as resource to implement nonlocal gates. In [59], the exact requirements that such a scheme can be applied were formulated, and in the following we assume that these requirements are met. In particular, the schemes we consider requires the realizability of the following operations: (i) (non-local) twosystem interactions that act only on specific virtual subsystems of each particle, e.g., $A_{2}, B_{2}$, without affecting other virtual subsystems; (ii) single-system measurement on one virtual subsystem without affecting other virtual subsystems; (iii) arbitrary unitary operations on one virtual subsystem; and (iv) (local) interactions between arbitrary virtual subsystems $A_{j}, A_{k}$. While (i) requires a specific type of two-body interactions between two systems $A$ and $B$, requirements (ii)-(iv) are concerned with the ability to locally manipulate a single system.

Similarly as in the context of quantum computation [59], here we consider the case that auxiliary qubits are used to generate and store the entangled states $\left|\psi_{E}\right\rangle$, and teleportation is used to generate time evolutions governed by a many-body Hamiltonian for time $\delta t^{\prime}$ on the storage qubits. Note that the involved measurements and manipulations are local, and only the generation of $\left|\psi_{E}\right\rangle$ requires non-local operations. In addition, if enough auxiliary levels $D \approx 8-32$ are available, entanglement purification can be applied to increase the fidelity of the noisy entangled states generated by noisy two-system interactions. The states $\left|\psi_{E}\right\rangle$ are essentially GHZ states (or graph states that are locally equivalent to GHZ states), and known multiparty entanglement purification protocols [55, 56] can be used to purify these states and to increase their fidelity. Consequently, many-body interaction Hamiltonians acting on storage qubits with increased fidelity can be generated.

\section{B. Perfect single-system operations}

In this section we assume that local operations are perfect, i.e. $p_{l}=1$ or $\gamma_{l}=\kappa_{l}=0$, and investigate the influence of noise resulting from two-system interactions in the generation of $\left|\psi_{E}\right\rangle$. In the case of $n$-body interactions that should be applied on (virtual) qubits $A_{1}, B_{1}, \ldots, N_{1}$, the state $\left|\psi_{E}\right\rangle$ is a $2 n+1$ qubit state given by

$$
\left|\psi_{E}\right\rangle=1 / \sqrt{2}\left(\left|\phi^{+}\right\rangle^{\otimes n}|0\rangle_{E}+\left|\phi^{-}\right\rangle^{\otimes n}|1\rangle_{E}\right),
$$

where the last qubit corresponds to an additional auxiliary system $E$ and the $\left|\phi^{+}\right\rangle$states corresponding to virtual qubits two and three of the same system, $X_{2} X_{3}$, $X \in\{A, B, \ldots, N\}$. It follows that $\left|\psi_{E}\right\rangle$, when considered as a $n+1$ system state, is up to local, single system operations (that include also local CNOT or phase gates which we however consider to be noiseless in this section), equivalent to a GHZ-type graph state

$$
\left|\tilde{\psi}_{E}\right\rangle=\left[1 / \sqrt{2}\left(|+\rangle^{\otimes n}|0\rangle_{E}+|-\rangle^{\otimes n}|1\rangle_{E}\right)\right] \otimes|0\rangle^{\otimes n} .
$$

The state $\left|\tilde{\psi}_{E}\right\rangle$ can be generated by applying phase gates $U_{P G}$ between the auxiliary particle $E$ and all other parties, $\prod_{X \in\{A, B, \ldots, N\}} U_{P G}^{(E X)}$. The Bell measurements involved in the teleportation process act on virtual qubits one and two of the same system, and are hence local, single system operation (which are considered to be noiseless in this section). Hence the only noise in the process comes from imperfect preparation of the state $\left|\tilde{\psi}_{E}\right\rangle$ which involves $n$ two-system gates.

An alternative scheme that uses a reduced number of virtual qubits per system (two rather than three) is the following. We use the $n+1$ qubit state $\left|\tilde{\psi}_{E}\right\rangle$ directly, and replace the (local) Bell measurements by local phase gates that couple the storage qubits $A_{1}, B_{1}, \ldots N_{1}$ to the qubits $A_{2}, B_{2}, \ldots N_{2}$ of the state $\left|\tilde{\psi}_{E}\right\rangle$, and measurements in the $\sigma_{x}$-basis on the storage qubits. Together, this again leads to transfer of quantum information to the system $A_{2}, \ldots, N_{2}$. An appropriate measurement on the ancilla qubit $E$, where the measurement direction again depends on the outcome of all previous $\sigma_{x}$ measurement, finally allows one to implement the operation $e^{-\alpha \sigma_{z}^{\otimes n}}$ for arbitrary $\alpha$ in the storage particles (now held in $A_{2}, \ldots, N_{2}$ ). Additional Pauli operations - depending on the results of the measurements- on the final state are required to adjust the local basis. This scheme has the advantage that fewer virtual qubits are involved. In fact, this procedure is equivalent to the way of obtaining $n$-qubit phase gates in one-way quantum computation put forward by Browne and Briegel in Ref. [46].

We remark that the auxiliary system $E$ may also be thought of being an additional virtual qubit of one of the systems particles, e.g. $A_{3}$ of system $A$. In this case, fewer two-system gates are required, and the influence of noise is lower, yielding to higher fidelities. However one of the systems needs to have twice as many levels as the other systems, and the process becomes non-symmetric. In the following, we will not consider this situation, but rather the symmetric situation were the auxiliary qubit is given by an additional, independent system particle, and we use the second scheme involving fewer virtual qubits for simplicity.

\section{Three-body interactions}

We start by considering three-body interactions, $U\left(\delta t^{\prime}\right)=e^{-\delta t^{\prime} \sigma_{z}^{\otimes 3}}$, i.e. $\alpha=\delta t^{\prime}$. We consider first the case of phase noise, and then the case of white noise in two-system interactions.

Phase noise:

We consider a basic evolution coupling two virtual qubits of two different systems that is governed by the Hamiltonian $H=\sigma_{z} \otimes \sigma_{z}$, and where the noise part 
is described by a Liouvillian that corresponds to single qubit dephasing, i.e. is given by Eq. [148. Noise part and Hamiltonian part commute, and thus the solution of the corresponding master equation is simple. A noisy phase gate can be obtained using additional single-qubit $z$-rotations, and one finds $\mathcal{E}_{P G} \rho=$ $\mathcal{D}^{(1)}(p) \mathcal{D}^{(2)}(p) U_{P G} \rho U_{P G}^{\dagger}$, where $p=p(t)=\exp \left(-\gamma_{0} \pi / 4\right)$ and $\mathcal{D}$ is a single-qubit dephasing map specified in Eq. 104

Generation of the state $\left|\tilde{\psi}_{E}\right\rangle$ using such noisy gates leads to a mixed state

$$
\rho_{E}=\mathcal{D}^{\left(A_{2}\right)}(p) \mathcal{D}^{\left(B_{2}\right)}(p) \mathcal{D}^{\left(C_{2}\right)}(p) \mathcal{D}^{(E)}\left(p^{3}\right)\left|\tilde{\psi}_{E}\right\rangle\left\langle\tilde{\psi}_{E}\right|(2
$$

Notice that $\left|\tilde{\psi}_{E}\right\rangle=U_{P G}^{\left(A_{2} E\right)} U_{P G}^{\left(B_{2} E\right)} U_{P G}^{\left(C_{2} E\right)}|++++\rangle$, and the phase gates commute with the noise processes. Also the phase gates $U_{P G}^{\left(A_{1} A_{2}\right)}, U_{P G}^{\left(B_{1} B_{2}\right)}, U_{P G}^{\left(C_{1} C_{2}\right)}$ used to couple the state $\left|\tilde{\psi}_{E}\right\rangle$ to the storage qubits, and the $\sigma_{x}$ measurements on storage qubits $A_{1}, B_{1}, C_{1}$, commute with the noise. Noise acting on the ancilla system $E$ transforms after the measurement to correlated phase noise acting on the remaining qubits $A_{2}, B_{2}, C_{2}$. That is, for an arbitrary input state $\rho$, the output state after the sequences of measurements and corresponding local correction operations is given by

$\mathcal{E} \rho=\mathcal{D}^{\left(A_{2}\right)}(p) \mathcal{D}^{\left(B_{2}\right)}(p) \mathcal{D}^{\left(C_{2}\right)}(p) \tilde{\mathcal{D}}^{\left(A_{2} B_{2} C_{2}\right)}\left(p^{3}\right) U\left(\delta t^{\prime}\right) \rho U\left(\delta t^{\prime}\right)^{\dagger}$,

where

$\tilde{\mathcal{D}}_{A B C}(p) \rho=p \rho+\frac{1-p}{2}\left(\rho+\sigma_{z}^{(A)} \sigma_{z}^{(B)} \sigma_{z}^{(C)} \rho \sigma_{z}^{(A)} \sigma_{z}^{(B)} \sigma_{z}^{(C)}\right)$,

and $U\left(\delta t^{\prime}\right)=e^{-\delta t^{\prime} \sigma_{z}^{\otimes 3}}$.

The fidelity of the noisy map $\mathcal{E}$ with respect to the ideal process $\hat{U}(\alpha)$ (where $\left.\hat{U} \rho=U \rho U^{\dagger}\right), F\left(\mathcal{E}, \hat{U}\left(\delta t^{\prime}\right)\right.$ ), can easily be calculated and one obtains

$$
\begin{aligned}
F\left(\mathcal{E}, \hat{U}\left(\delta t^{\prime}\right)\right) & =\left(\frac{1+p}{2}\right)^{3} \frac{1+p^{3}}{2}+\left(\frac{1-p}{2}\right)^{3} \frac{1-p^{3}}{2} \\
& =\left(\frac{1+p^{2}}{2}\right)^{3} \\
& \approx 1-3 \pi / 4 \gamma_{0}
\end{aligned}
$$

where the last equality only holds for $\gamma_{0} \pi / 4 \ll 1$. The local noise equivalent $\gamma$ can be determined under the previous assumptions, and one finds

$$
\gamma \approx \frac{\pi}{2 \delta t^{\prime}} \gamma_{0}
$$

Note that the local noise equivalent is of the same order of magnitude as in the case of graph state encoding (see Eq. 185), and the fidelity is constant, independent of $\delta t^{\prime}$.

White noise:

A similar analysis can be performed for white noise. In this case, noise part and unitary part do not commute, and the influence of noise on the ancilla qubit leads to a slightly different noise process on the final system. We find

$$
\mathcal{E} \rho=\tilde{\mathcal{M}}^{\left(A_{2} B_{2} C_{2}\right)}\left(p^{3}\right) \mathcal{M}^{\left(A_{2}\right)}(p) \mathcal{M}^{\left(B_{2}\right)}(p) \mathcal{M}^{\left(C_{2}\right)}(p) \rho,
$$

where

$$
\begin{aligned}
\tilde{\mathcal{M}}^{(A B C)}(p) \rho & =p U\left(\delta t^{\prime}\right) \rho U\left(\delta t^{\prime}\right)^{\dagger} \\
& +\frac{1-p}{2}\left(\rho+\sigma_{z}^{(A)} \sigma_{z}^{(B)} \sigma_{z}^{(C)} \rho \sigma_{z}^{(A)} \sigma_{z}^{(B)} \sigma_{z}^{(C)}\right),
\end{aligned}
$$

with $U\left(\delta t^{\prime}\right)=e^{-\delta t^{\prime} \sigma_{z}^{\otimes 3}}$, and $\mathcal{M}(p)$ is a depolarizing map specified in Eq. 105 with $p=\exp \left(-\kappa_{0} \pi / 4\right)$. It follows that the fidelity of the process with respect to the ideal operation $\hat{U}\left(\delta t^{\prime}\right)$ can be estimated to be

$$
\begin{aligned}
F\left(\mathcal{E}, \hat{U}\left(\delta t^{\prime}\right)\right) & =\left(\frac{1+3 p}{4}\right)^{3} \frac{1+p^{3}}{2}+\left(\frac{1-p}{4}\right)^{3} \frac{1-p^{3}}{2} \\
& \approx 1-\frac{15 \pi}{16} \kappa_{0},
\end{aligned}
$$

and for the local noise equivalent $\kappa$ one finds for $\delta t^{\prime} \pi / 4 \ll$ 1

$$
\kappa \approx \frac{5 \pi}{12 \delta t^{\prime}} \kappa_{0}
$$

\section{Many-body interactions}

It is straightforward to perform the analysis for $n$ qubit operations $U\left(\delta t^{\prime}\right)=e^{-\delta t^{\prime} \sigma_{z}^{\otimes n}}$. In this case, a $n+1$ qubit GHZ state is generated by noisy two-body interactions, and processed by performing local operations and measurements. In the case of phase noise, one finds a fidelity

$$
\begin{aligned}
F\left(\mathcal{E}, \hat{U}\left(\delta t^{\prime}\right)\right) & =\left(\frac{1+p}{2}\right)^{n} \frac{1+p^{n}}{2}+\left(\frac{1-p}{2}\right)^{n} \frac{1-p^{n}}{2} \\
& \approx 1-n \pi / 4 \gamma_{0},
\end{aligned}
$$

where the last equality only holds for $\gamma_{0} \pi / 4 \ll 1$. For the local noise equivalent one finds $\gamma \approx \frac{\pi}{2 \delta t^{\prime}} \gamma_{0}$.

The corresponding expressions for white noise, where $p=\exp \left(-\kappa_{0} \pi / 4\right)$, are

$$
\begin{aligned}
F\left(\mathcal{E}, \hat{U}\left(\delta t^{\prime}\right)\right) & =\left(\frac{1+3 p}{4}\right)^{n} \frac{1+p^{n}}{2}+\left(\frac{1-p}{4}\right)^{n} \frac{1-p^{n}}{2} \\
& \approx 1-\frac{5 n \pi}{16} \kappa_{0},
\end{aligned}
$$

and the local noise equivalent is given by $\kappa=\frac{5 \pi}{12 \delta t^{\prime}} \kappa_{0}$.

\section{Timing errors}

Analyzing timing errors for this teleportation protocol is particularly easy and has many similarities to the dephasing case. Like the dephasing case, we will examine 
the situation where there are no local errors and that all noise in the system comes from the generation of the GHZ state $\left|\tilde{\psi}_{E}\right\rangle$, which is generated via phase gates. As we saw above, these phase gates can be generated by a two qubit Ising interaction Hamiltonian, $H=\sigma_{z} \otimes \sigma_{z}$. Here we examine the effect of timing errors on this entangling Hamiltonian. The action of our noisy phase gate on an arbitrary state is defined as,

$$
\mathcal{E}_{P G} \rho=\mathcal{T} U_{P G} \rho U_{P G}^{\dagger},
$$

where $\mathcal{T}$ is defined in Eq. 122, The map $\mathcal{T}$ is a correlated two-qubit dephasing channel. We note that $\mathcal{T}$ will commute with the coherent processes that generate $|\tilde{\psi}\rangle$, this sibilantly simplifies our analysis. For clarity, we will first consider the generation of three qubit interaction. If we attempt to generate $\left|\tilde{\psi}_{E}\right\rangle$ with these gates the resulting state will be,

$$
\rho_{E}=\mathcal{T}^{\left(A_{2} E\right)} \mathcal{T}^{\left(B_{2} E\right)} \mathcal{T}^{\left(C_{2} E\right)}\left|\tilde{\psi}_{E}\right\rangle\left\langle\tilde{\psi}_{E}\right|
$$

Now, what is the effect of attempting our teleportationbased Hamiltonian simulation protocol using $\rho_{E}$ as a resource? It is easy to see that noise process commutes with the $\sigma_{x}$ measurements on the storage qubits $A_{1}, B_{1}$, and $C_{1}$. The only non-trivial transformation is as a result of the projective measurement on qubit $E$. We saw above that, the effect of measuring a $E$ in the basis that we have chosen causes a dephasing channel acting on $E$ to transform into a three-qubit dephasing channel acting on qubits $A_{2}, B_{2}$, and $C_{2}$. If we instead had a correlated phase error between $E$ and $A_{2}$ before applying the necessary measurement on $E$ we will have applied a phase flip on qubit $A_{2}$ followed by the same three-qubit correlated error as above. The effect of this operation on our protocol is to create a two qubit correlated dephasing operation on qubits $B_{2}$ and $C_{2}$. That is, the timing noise map $\mathcal{T}^{\left(A_{2} E\right)}$ gets transformed under this teleportation protocol into $\mathcal{T}^{\left(B_{2} C_{2}\right)}$. Considering this, the operation induced by the teleportation protocol on an arbitrary input $\rho$ is given by,

$$
\mathcal{E} \rho=\mathcal{T}^{\left(A_{2} B_{2}\right)} \mathcal{T}^{\left(A_{2} C_{2}\right)} \mathcal{T}^{\left(B_{2} C_{2}\right)} \hat{U}\left(\delta t^{\prime}\right) \rho .
$$

Because the noise process $\mathcal{T}^{\left(A_{2} B_{2}\right)} \mathcal{T}^{\left(A_{2} C_{2}\right)} \mathcal{T}^{\left(B_{2} C_{2}\right)}$ commutes with $\hat{U}\left(\delta t^{\prime}\right)$,

$$
D\left(\mathcal{E}, \hat{U}\left(\delta t^{\prime}\right)\right)=D\left(\mathcal{T}^{\left(A_{2} B_{2}\right)} \mathcal{T}^{\left(A_{2} C_{2}\right)} \mathcal{T}^{\left(B_{2} C_{2}\right)}, \mathbb{1}\right)
$$

and

$$
F\left(\mathcal{E}, \hat{U}\left(\delta t^{\prime}\right)\right)=F\left(\mathcal{T}^{\left(A_{2} B_{2}\right)} \mathcal{T}^{\left(A_{2} C_{2}\right)} \mathcal{T}^{\left(B_{2} C_{2}\right)}\right)
$$

Thus If we now make the assumption that the timing error is gaussian distributed, as we did in Section VII we can calculate the resulting fidelity to be

$$
F\left(\mathcal{E}, \hat{U}\left(\delta t^{\prime}\right)\right)=\left(\frac{1+q}{2}\right)^{3}+\left(\frac{1-q}{2}\right)^{3}
$$

Generalizing this analysis for the generation of the $n$ qubit operations like $U\left(\delta t^{\prime}\right)=e^{-i^{\prime} \delta t^{\prime} \sigma_{z}^{\otimes n}}$ is straightforward. As a resource we consider an $n+1$ qubit GHZ state that is generated by phase gates as in the previous section. Given an arbitrary input $\rho$ the effect of performing the teleportation protocol ideally is to output the state $\hat{U}\left(\delta t^{\prime}\right) \rho$ at qubits $A_{2} B_{2} \ldots N_{2}$. Performing the same analysis as above and noting that of measuring $E$ is to transform operations like $\mathcal{T}_{A_{2} E}$ into operations like $\mathcal{T}_{B_{2} C_{2} \ldots N_{2}}$ we find that the effect of timing errors is to induce the following map

$$
\mathcal{E} \rho=\mathcal{T}^{\left(A_{2} B_{2} \ldots N_{2}\right)} \mathcal{T}^{\left(A_{2} C_{2} \ldots N_{2}\right)} \mathcal{T}^{\left(B_{2} C_{2} \ldots N_{2}\right)} \ldots \hat{U} \delta t^{\prime} \rho
$$

The resulting fidelity is,

$$
F\left(\mathcal{E}, \hat{U}\left(\delta t^{\prime}\right)\right)=\left(\frac{1+q}{2}\right)^{n}+\left(\frac{1-q}{2}\right)^{n}
$$

\section{Entanglement purification and the influence of local noise}

In a similar way, one can also take the influence of noise in local (i.e. single system) operations into account. In this case, the coupling of system particles to the auxiliary GHZ state is also noisy, leading to a further reduction in the fidelity of the final process. The corresponding noise processes are described by similar master equations as we use for modelling of two-system interactions, however the coupling parameters $\kappa_{l}$ or $\gamma_{l}$ describing the noise level are different. As indicated in the discussion at the beginning of this section, we will assume that single system noise is smaller than noise in two system operations, i.e. $\kappa_{l} \ll \kappa_{0}$ and $\gamma_{l} \ll \gamma_{0}$.

In the case of phase noise, we have shown that the final state is given by Eq. 228 when assuming that local single system operations are perfect, while the influence of noisy single system operations is essentially covered by additional noise maps maps $\mathcal{D}^{\left(A_{2}\right)}\left(p_{l}^{2}\right), \mathcal{D}^{\left(B_{2}\right)}\left(p_{l}^{2}\right), \mathcal{D}^{\left(C_{2}\right)}\left(p_{l}^{2}\right)$ with $p_{l}=\exp \left(-\gamma_{l} \pi / 4\right)$. Under the assumption that $\gamma_{l} \ll \gamma_{0}$, the final fidelity and local noise equivalent are essentially determined by $\gamma_{0}$, with small corrections due to additional single system noise. A similar situation is encountered in the case of depolarizing noise.

We will not further go into details about the influence of local noise in the standard protocol, but rather consider the case where multipartite entanglement purification [55, 55] is used to produce high-fidelity resource states $\left|\tilde{\psi}_{E}\right\rangle$ and hence to generate many-body interactions with high fidelity. That is, we assume that systems with additional auxiliary levels - which are used for storage and purification of entangled resource states - are available. In this case, noise in local operations determines the achievable fidelity of the resource states, and hence the final fidelity of the many-body interaction. In particular, the resulting fidelity is independent of amount of noise in two-system operations and hence independent of $\kappa_{0}, \gamma_{0}$, provided the noise is sufficiently small such 
that entanglement purification can be successfully applied. For perfect single system operations, the tolerable amount of noise in the two system operations is up to $66 \%$ [59] (or slightly smaller when using direct multiparticle entanglement purification) when considering depolarizing noise. Non-zero amount of noise in single system operations leads to a slightly smaller error tolerance, however as long as $p_{l}=\exp \left(-\kappa_{l} \pi / 4\right)$ is sufficiently close to one (errors at the order of percent, i.e. $p_{l} \approx 0.99$ ) we find that noise in two-system operations of the order of several tens of percent can still be tolerated, without influencing the final achievable fidelity of the purified state and the resulting many-body interaction.

An exact analytic treatment of the total process is difficult due to difficulties in analytically describing multiparticle entanglement purification protocols and the reachable fidelities of such schemes. We have thus performed a numerical simulation taking all errors due to imperfect operations into account. The results are presented in the following.

\section{Three-body interactions}

For three-body interactions, the required resource state is a GHZ state of four qubits, which can be purified using the entanglement purification protocol introduced in Ref. [55, 56]. The fidelity of the resulting three-body interaction $\exp \left(-\delta t^{\prime} \sigma_{z}^{\otimes 3}\right)$ is plotted in Fig. 9 for $\delta t^{\prime} \ll 1$ ( $\delta t^{\prime}=\pi / 2^{15}$ to be precise) and for a three-qubit gate with $\delta t^{\prime}=\pi / 4$. As can be seen from the plot, the resulting fidelity is almost identical for the two cases, i.e. to a large extend independent of $\delta t^{\prime}$. The plot shows the dependence of (one minus) the fidelity on the noise in local operation $1-p_{l}$, where $p_{l}=\exp \left(-\kappa_{l} \pi\right)$ here. Notice the linear scaling in the log-log plot.

We remark the local noise equivalent has no useful meaning in this context, as the resulting fidelity of the many-body interaction is independent of noise in twosystem operations (the reachable fidelity of entanglement purification only depends on noise in local single system operations). More precisely, for any noise parameter $\kappa_{0}$ (describing coupling strength to environment for twosystem operations) that is sufficiently small such that entanglement can be generated by the resulting twosystem operation, we obtain the same local noise equivalent $\kappa$ which only depends on $\kappa_{l}$, the noise parameter for local single system operations. Notice that $\kappa$ can be significantly smaller than the initial noise parameter $\kappa_{0}$. For perfect local control operations and sufficiently many auxiliary levels, we can even obtain $\kappa=0$.

\section{Many-body interactions}

For $n$-body interactions, the required resource state is a GHZ state of $n$ qubits. Fig. 10 shows the reachable fidelity of the $n+1$-qubit GHZ states for different values

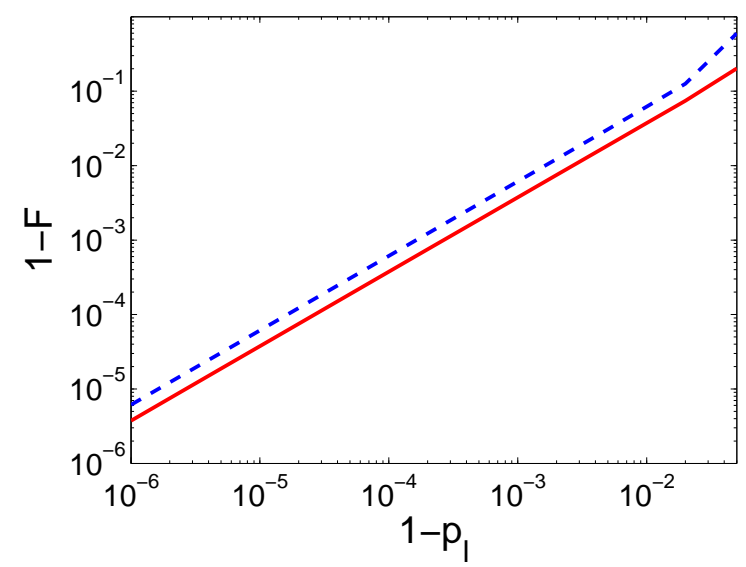

FIG. 9: Fidelity of three-body interaction $U=\exp \left(-\delta t^{\prime} \sigma_{z}^{\otimes 3}\right)$ for time $\delta t^{\prime}=\pi / 2^{15} \approx 10^{-4}$ (red, solid) and $\delta t^{\prime}=\pi / 4$ (blue, dashed) using teleportation based method. One minus fidelity is plotted as a function of noise in local operation $1-p_{l}$, where $p_{l}=\exp \left(-\kappa_{l} \pi\right)$ and $1-p_{l}=0$ corresponds to perfect local operations leading to unit fidelity.

of noise in local control operation $1-p_{l}$. For $n=3,4$ also the fidelity of resulting $n$-qubit interaction $\exp \left(-\delta t^{\prime} \sigma_{z}^{\otimes n}\right)$ for $\delta t^{\prime}=\pi / 2^{15}$ is shown, which is of the same order of magnitude as the fidelity of the purified GHZ state.

\section{COMPARISON OF METHODS}

In this section, we will recall and compare the main properties of the different methods to generate manybody interactions. We consider many-body interactions $\exp \left(-i \delta t^{\prime} \sigma_{z}^{\otimes n}\right)$ generated by the commutator method, graph state encoding or teleportation based method with entanglement purification.

In the commutator method, the fidelity increases for smaller $\delta t^{\prime}$, even though the local noise equivalent increases. When implementing a gate (i.e. a certain interaction for time $t=O(1)$ ) by sequentially applying interactions $\exp \left(-i \delta t^{\prime} \sigma_{z}^{\otimes n}\right)$ generated this way, there is an optimal $\delta t^{\prime}$. The fidelity of such a gate is significantly lower than the fidelity of a many-body interaction applied for short time $\delta t^{\prime} \ll 1$.

For graph state encoding, in contrast, the fidelity is constant and independent of $\delta t^{\prime}$. The fidelity is determined by applications of noisy two-body interactions for times $t=O(1)$ (the graph state encoding), leading to a significantly lower fidelity for small $\delta t^{\prime}$ as compared to the commutator method. The local noise equivalent also increases with decreasing $\delta t^{\prime}$, faster as for the commutator method. However, also gates or evolutions for times $t \gg 1$ can be simulated with same accuracy, leading to an advantage of graph state encoding method over com- 


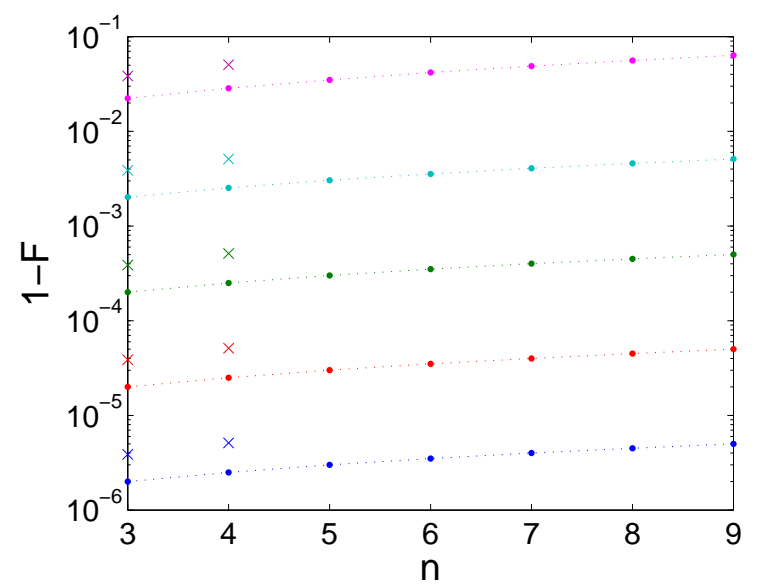

FIG. 10: Reachable fidelity to generate $n+1$ qubit GHZ states using multiparticle entanglement purification (dots), and to generate $n$-body interactions of the form $\exp \left(-\delta t^{\prime} \sigma_{z}^{\otimes n}\right)$ from the GHZ states $(\times)$ following the protocol outlined in main text. One minus fidelity is plotted against $n$. Data points/curves from top to bottom correspond to noise parameter for local operation $\left(1-p_{l}\right)=10^{-2}, 10^{-3}, 10^{-4}, 10^{-5}, 10^{-6}$ respectively, where $p_{l}=\exp \left(-\kappa_{l} \pi\right)$.

mutator method in such situations.

The teleportation based method making use of auxiliary local degrees of freedom also leads to a constant fidelity, almost independent of $\delta t^{\prime}$, and hence also gates with $t=O(1)$ can be implemented with same fidelity. However, for the teleportation based method using entanglement purification, the fidelity is determined by the noise in local single system operations rather than noise in two-system interactions, possibly leading to much better accuracies than reachable with graph state encoding or the commutator method. Only for $\delta t^{\prime} \ll 1$ the commutator method may be more accurate, and for $\delta t^{\prime} \gg 1$ (fixed) graph state encoding may be favorable. The advantage of the teleportation based method seems to increase when considering the implementation of $n$-body interactions with increasing $n$. It follows that the teleportation based method, together with entanglement purification, provides a possibility to successfully simulate interacting high-dimensional quantum system or systems with many-body interactions with high accuracy. This can still be seen as an intermediate stage to full scale, universal fault tolerant quantum computation, and should be significantly easier to implement than the latter.

\section{SUMMARY AND CONCLUSIONS}

We have introduced and investigated several methods to generate many-body interactions from two-body interactions. We reviewed the standard commutator method which is based on usage of higher order terms in the Trotter-Suzuki expansion. We have shown how to use unitary conjugation or graph state encoding to generate large classes of many-body interaction Hamiltonians when using a fixed encoding, and arbitrary Hamiltonians when allowing for a variable encoding. Several examples, including three-body Hamiltonians with phase transition, simulation of interacting $d$-level systems and generation of plaquette interactions have been put forward. Furthermore, we have investigated the usage of maximally entangled states produced by two-body interactions to generate many-body interactions by means of teleportation, i.e. using teleportation based methods.

We have studied the influence of noise, described by generic error models, on the simulation process for the three schemes discussed above. For long-time simulations, the fixed graph state encoding method is favorable.

However, if one has access to auxiliary systems (e.g. higher dimensional systems), then one may use them to reduce noise. In particular, one can use higher dimensional systems to simulate lower dimensional systems, and use auxiliary levels to perform entanglement purification. This makes the teleportation based method a possible solution to overcome, or at least significantly reduce, the influence of noise on a simulation process. While this method makes use, to a certain extent, of elements of measurement based quantum computation, it still does not make use of the whole blown up machinery of a full fault tolerant quantum computer. We believe that our considerations are useful with respect to the ongoing effort to design and eventually use quantum simulators based on well controllable, but nevertheless noisy quantum systems.

\section{Acknowledgements}

M.J.B. would like to thank Otfried Gühne, Gavin Brennen, Dan Browne, Ken Brown, Chris Dawson, and Henry Haselgrove for helpful discussions.

This work was supported by the Austrian Science Foundation (FWF), the European Union (OLAQUI,SCALA,QICS) and the Austrian Academy of Sciences (ÖAW) through project APART (W.D.).
[1] R. P. Feynman, Int. J. Theor. Phys. 21, 467 (1982).

[2] D. S. Abrams and S. Lloyd, Phys. Rev. Lett. 79, 2586 (1997).

[3] D. W. Berry, G. Ahokas, R. Cleve, and B. C.
Sanders, Comm. in Math. Phys. 270, 359 (2007), arXiv quant-ph/0508139v2.

[4] A. M. Childs, Phd thesis, MIT (2004).

[5] J. L. Dodd, M. A. Nielsen, M. J. Bremner, and 
R. T. Thew, Phys. Rev. A. 65, 040301(R) (2002), arXiv quant-ph/0106064.

[6] M. J. Bremner, J. L. Dodd, M. A. Nielsen, and D. Bacon, Phys. Rev. A 69, 012313 (2003), arXiv quant-ph/0307148.

[7] M. J. Bremner, D. Bacon, and M. A. Nielsen, Phys. Rev. A 71, 052312 (2005).

[8] P. Wocjan, M. Roetteler, D. Janzing, and T. Beth, Quantum Information and Computation 2, 133 (2002), arXiv quant-ph/0109063.

[9] P. Wocjan, M. Rötteler, D. Janzing, and T. Beth, Phys Rev. A. 65, 042309 (2002), arXiv quant-ph/0109088.

[10] C. H. Bennett, J. I. Cirac, M. S. Leifer, D. W. Leung, N. Linden, S. Popescu, and G. Vidal, Phys. Rev. A. 66, 012305 (2002), arXiv quant-ph/0107035.

[11] W. Dür, G. Vidal, J. I. Cirac, N. Linden, and S. Popescu, Phys. Rev. Lett. 87, 137901 (2001), arXiv quant-ph/0006034.

[12] H. L. Haselgrove, M. A. Nielsen, and T. J. Osborne, Phys. Rev. A. 68, 042303 (2003), arXiv quant-ph/0303070.

[13] G. Vidal and J. I. Cirac, Phys. Rev. A 66, 022315 (2002), arXiv quant-ph/0108076

[14] E. Jane, G. Vidal, W. Dür, P. Zoller, and J. I. Cirac, Quantum Information and Computation 3, 15 (2003), quant-ph/0207011.

[15] S. S. Bullock, G. K. Brennen, and D. P. O'Leary, Journal of Mathematical Physics 46, 062104 (2005), arXiv quant-ph/0402051.

[16] V. Murg and J. I. Cirac, Phys. Rev. A 69, 042320 (2004).

[17] D. Jaksch, C. Bruder, J. I. Cirac, C. W. Gardiner, and P. Zoller, Phys. Rev. Lett. 81, 3108 (1998).

[18] D. Jaksch and P. Zoller, Annals of Physics 315, 52 (2005), cond-mat/0410614.

[19] A. Micheli, G. K. Brennen, and P. Zoller, Nature Physics 2, 341 (2006), arXiv quant-ph/0512222

[20] F. Verstraete and J. I. Cirac, J.Stat.Mech. 09012 (2005), cond-mat/0508353.

[21] G. Ortiz, J. E. Gubernatis, E. Knill, and R. Laflamme, Phys. Rev. A 64, 022319 (2001).

[22] P. Jordan and E. Wigner, Z. Phys. 47, 631 (1928).

[23] J. Kempe, A. Kitaev, and O. Regev, SIAM Journal of Computing 35, 1070 (2006), arXiv.org quant-ph/0406180

[24] S. P. Jordan, E. Farhi, and P. W. Shor, Phys. Rev. A 74, 052322 (2006), quant-ph/0512170.

[25] K. R. Brown, R. J. Clark, and I. L. Chuang, Phys. Rev. Lett. 97, 050504 (2006), arXiv quant-ph/0601021.

[26] A. W. Harrow and M. A. Nielsen, Phys. Rev. A 68, 012308 (2003), arXiv quant-ph/0301108.

[27] M. A. Nielsen and I. L. Chuang, Quantum computation and quantum information (Cambridge University Press, Cambridge, 2000).

[28] R. Raussendorf, Phd thesis, Ludwig-Maximillians Universität München (2003).

[29] M. A. Nielsen and C. M. Dawson, Phys. Rev. A 71, 042323 (2005), arXiv quant-ph/0405134

[30] P. Aliferis and D. Leung, Phys. Rev. A. 73, 032308 (2006).

[31] C.M. Dawson, H.L. Haselgrove, and M.A. Nielsen, Phys. Rev. Lett. 96, 020501 (2006).

[32] R. Rausendorf and J. Harrington, Phys. Rev. Lett. 98, 190504 (2007).
[33] R. Rausendorf, J. Harrington, and K. Goyal, arXiv:quant-ph/0703143 (2007).

[34] D. Aharonov, A. Kitaev, and J. Preskill, Phys. Rev. Lett. 96, 050504 (2006), arXiv.org:quant-ph/0510231

[35] S. Virmani, S. F. Huelga, and M. B. Plenio, Phys. Rev. A 71, 042328 (2005)

[36] M. Hein, W. Dür W, J. Eisert, R. Raußendorf, M. Van den Nest and H.-J. Briegel H J, In Quantum computers, algorithms and chaos, International School of Physics Enrico Fermi, vol. 162, eds. G. Casati, D. Shepelyansky, P. Zoller and G. Benenti (Amsterdam: IOS Press, 2006); see also E-print: quant-ph/0602096

[37] J. K. Pachos and E. Rico, Phys. Rev. A 70, 053620 (2004), arXiv quant-ph/0404048.

[38] J. K. Pachos and M. B. Plenio, Phys. Rev. Lett. 93, 056402 (2004), arXiv quant-ph/0401106

[39] A. Kay, D. K. K. Lee, J. K. Pachos, M. B. Plenio, M. E. Reuter, and E. Rico, Optics and Spectroscopy 99, 355 (2005), arXiv quant-ph/0407121.

[40] F. Verstraete, M. Popp and J. I. Cirac, Phys. Rev. Lett. 92, 027901 (2004).

[41] M. Hermele, M. P. A. Fisher, and L. Balents, Phys. Rev. B 69, 064404 (2004).

[42] H. P. Büchler, M. Hermele, S. D. Huber, M. P. A. Fisher, and P. Zoller, Phys. Rev. Lett. 95, 040402 (2005).

[43] A. Jamiołkowski, Rep. Mod, Phys. 3, 275 (1972).

[44] J. I. Cirac, W. Dür, B. Kraus, and M. Lewenstein, Phys. Rev. Lett. 86, 544 (2001).

[45] J. de Pillis, Pacific Journal of Mathematics 23, 129 (1967).

[46] D. E. Browne and H. J. Briegel, in Lectures on Quantum Information, Eds. D. Bruss and G. Leuchs, Wiley-VCH, Berlin 2006; E-print: quant-ph/0603226.

[47] R. Raussendorf, D. E. Browne, and H. J. Briegel, Phys. Rev. A 68, 022312 (2003), quant-ph/0301052.

[48] R. Raussendorf and H. J. Briegel, Phys. Rev. Lett. 86, 5188 (2001).

[49] W. Dür, M. Hein, J. I. Cirac, and H. J. Briegel, Phys. Rev. A 72, 052326 (2005), arXiv quant-ph/0507134.

[50] W. Dür and J. I. Cirac, Phys. Rev. A 64, 012317 (2001).

[51] P. Aliferis and D. W. Leung, Phys. Rev. A 70, 062314 (2004).

[52] D. Gottesman and I. L. Chuang, Nature 402, 390 (1999), arXiv:quant-ph/9908010.

[53] M. Murao, M. B. Plenio, S. Popescu, V. Vedral, and P. L. Knight, Phys. Rev. A 57, R4075 (1998).

[54] E. N. Maneva and J. A. Smolin, in Quantum Computation and Quantum Information, edited by J. Samuel J. Lomonaco (American Mathemathical Society, Providence, RI, 2002), Vol. 305 of AMS Contempurary Mathematics; see also E-print quant-ph/0003099

[55] W. Dür, H. Aschauer, and H. J. Briegel, Phys. Rev. Lett. 91, 107903 (2003).

[56] H. Aschauer, W. Dür, and H. J. Briegel, Phys. Rev. A 71, 012319 (2005).

[57] H.J. Briegel and B.G. Englert, Phys. Rev. A 47, 3311 (1993).

[58] A. Gilchrist, N. K. Langford, and M. A. Nielsen, Phys. Rev. A 71, 062310 (2005).

[59] W. Dür and H.-J. Briegel, Phys. Rev. Lett. 90, 067901 (2003). 